University of Louisville

ThinkIR: The University of Louisville's Institutional Repository

Electronic Theses and Dissertations

8-2021

\title{
Vestiges and dry-erase ghosts.
}

Isaac Barzso

University of Louisville

Follow this and additional works at: https://ir.library.louisville.edu/etd

Part of the Composition Commons

\section{Recommended Citation}

Barzso, Isaac, "Vestiges and dry-erase ghosts." (2021). Electronic Theses and Dissertations. Paper 3651. https://doi.org/10.18297/etd/3651

This Master's Thesis is brought to you for free and open access by ThinkIR: The University of Louisville's Institutional Repository. It has been accepted for inclusion in Electronic Theses and Dissertations by an authorized administrator of ThinkIR: The University of Louisville's Institutional Repository. This title appears here courtesy of the author, who has retained all other copyrights. For more information, please contact thinkir@louisville.edu. 


\title{
Vestiges and Dry-Erase Ghosts
}

for two voices and ensemble

Isaac Barzso

B.M., Florida State University, 2019

A Thesis

Submitted to the Faculty of the

School of Music of the University of Louisville

In Partial Fulfillment of the Requirements

For the Degree of

\author{
Master of Music \\ in Music Composition
}

School of Music

University of Louisville

Louisville, KY

May 202I 


\section{Vestiges and Dry-Erase Ghosts}

for two voices and ensemble

Isaac Barzso

B.M., Florida State University, 2019

\section{A Thesis approved on}

April 29, 202I

by the following Thesis Committee

Krzysztof Wolek 


\section{ACKNOWLEDGMENTS}

To Krzysztof Wolek, for your guidance, encouragement, and advice in all situations and circumstances, as well as much-needed patience and belief as I followed my rabbit holes of concepts and experimentation -

to Katherine Donner and Steve Rouse, for your assistance and support as members of my thesis committee to the many professors and mentors whose willingness to challenge me over the last few years has held such significant influence over the development of this piece of music: Allison Ogden, Matilda Ertz, Matt Ertz, Christopher Brody, Kirsten Carithers, Ladislav Kubík, and Stephen Montague -

to my colleagues and friends at the University of Louisville who have been as equally willing to press me intellectually as they have been to share a drink: especially Rron Karahoda, Tanner Jones, Isaac Smith, Roger Knight, Rachael Smith, and Joshua Baerwald -

to my family for their love and unconditional support of my goals -

thank you. 


\section{PROGRAM NOTE}

vestiges and dry-erase ghosts comes heavily from the desire to explore the transfer of information and communication across media, coupled with an interest in focusing on changes over the course of long-term repetitive structures (through both literal repetition and re-inscription of material). A pre-existing semantic text is eschewed in favor of individual phonemes and indistinct vocalisms, balanced by processes of removal, repetitions, and cross-body transfer. Individual vocalisms are analyzed and split into multiple pieces of text, actions are removed aurally yet remain visually, and formal structures are interrupted by processes of repetition, with the long-form structure of the piece ultimately being built through the analysis of the details of which it is comprised.

Throughout the piece, the very idea of individual role and the dynamics of cause and effect are questioned. How do processes decay the whole before becoming re-inscribed? How do individual parts comprise a whole? What remains when we remove something from the whole? 


\section{TABLE OF CONTENTS}

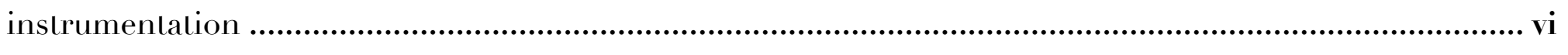

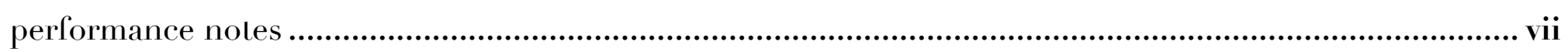

vestiges and dry-erase ghosts

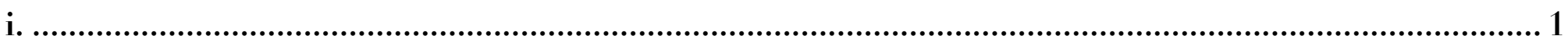

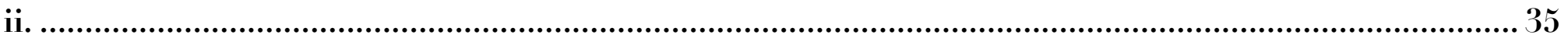

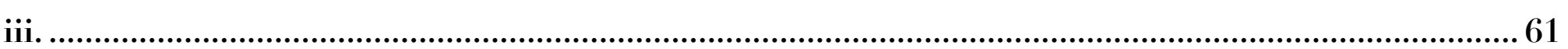

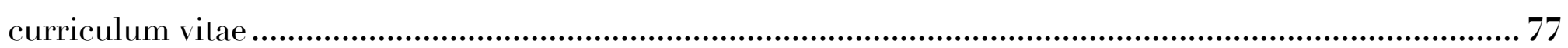




\section{INSTRUMENTATION}

flute

oboe

clarinet (doubling bass clarinet)

alto saxophone

horn in $\mathrm{f}$

trumpet in $\mathrm{c}$

trombone

two percussionists*

electric guitar

piano

two mezzo-sopranos

two violins

viola

violoncello

double bass

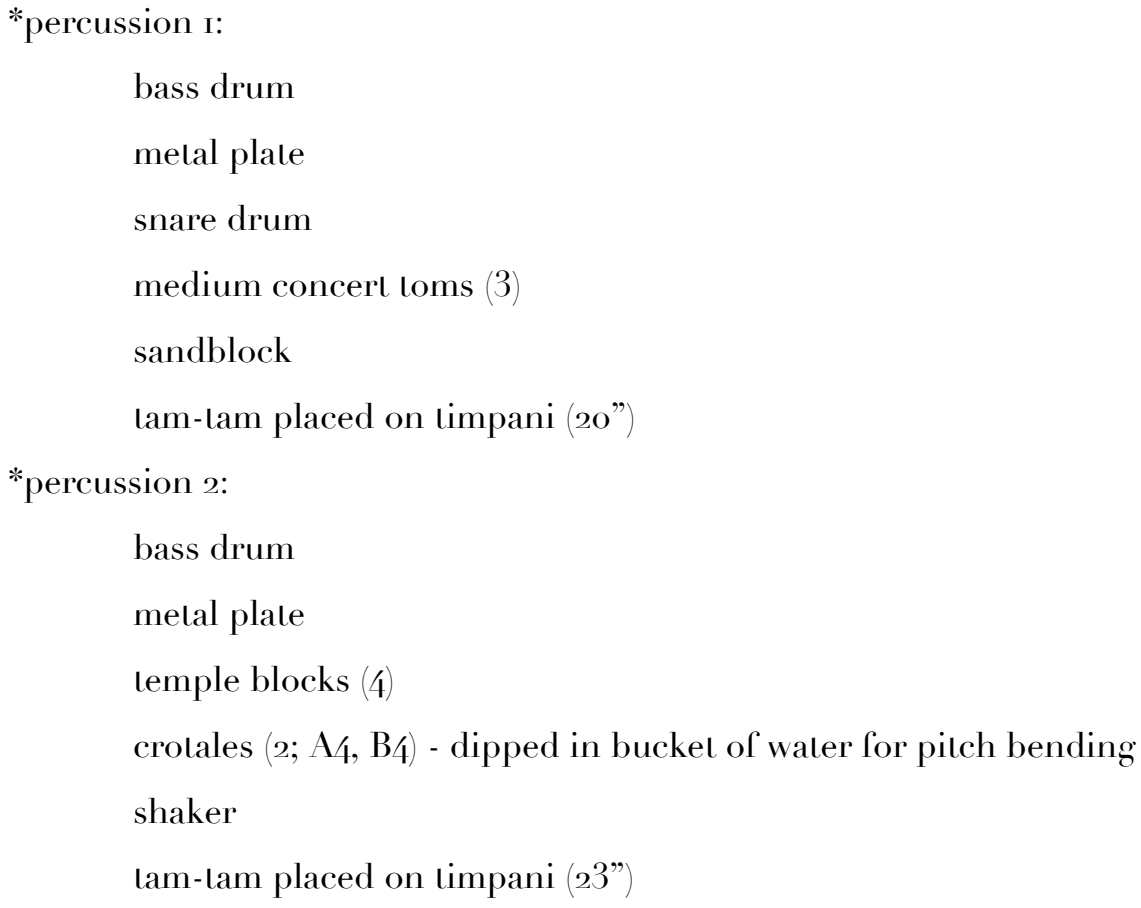

duration: ca. 24 minutes 


\section{performance notes}

all instruments

\section{$\mathrm{I} / 8$ and $\mathrm{I} / 4$-tone accidentals}
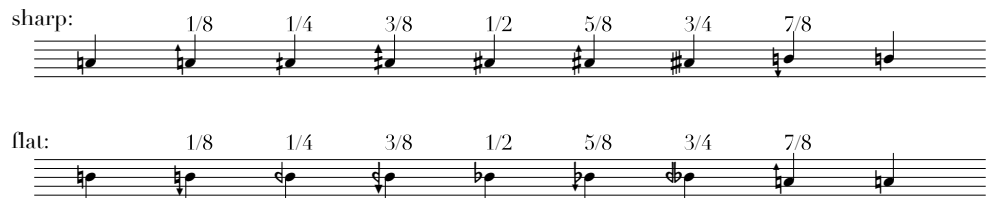

$\longrightarrow \quad$ transitions between performance techniques are indicated by an arrow

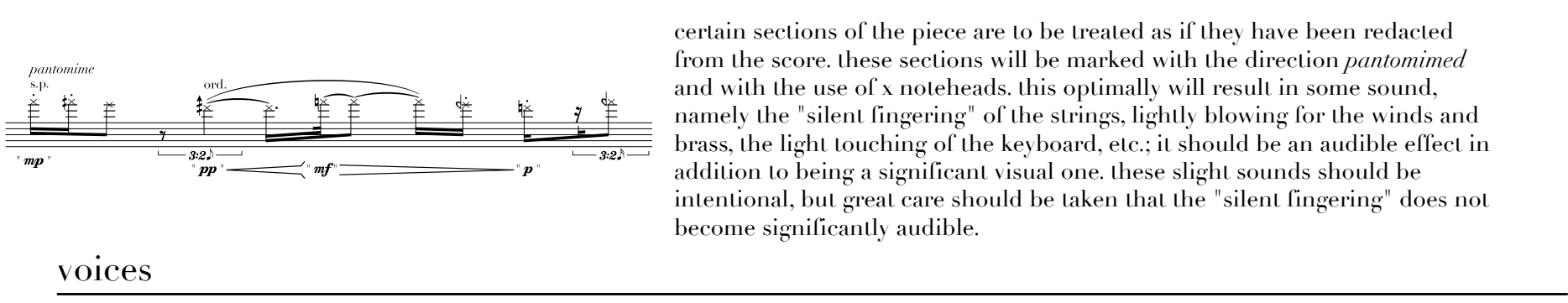

voices

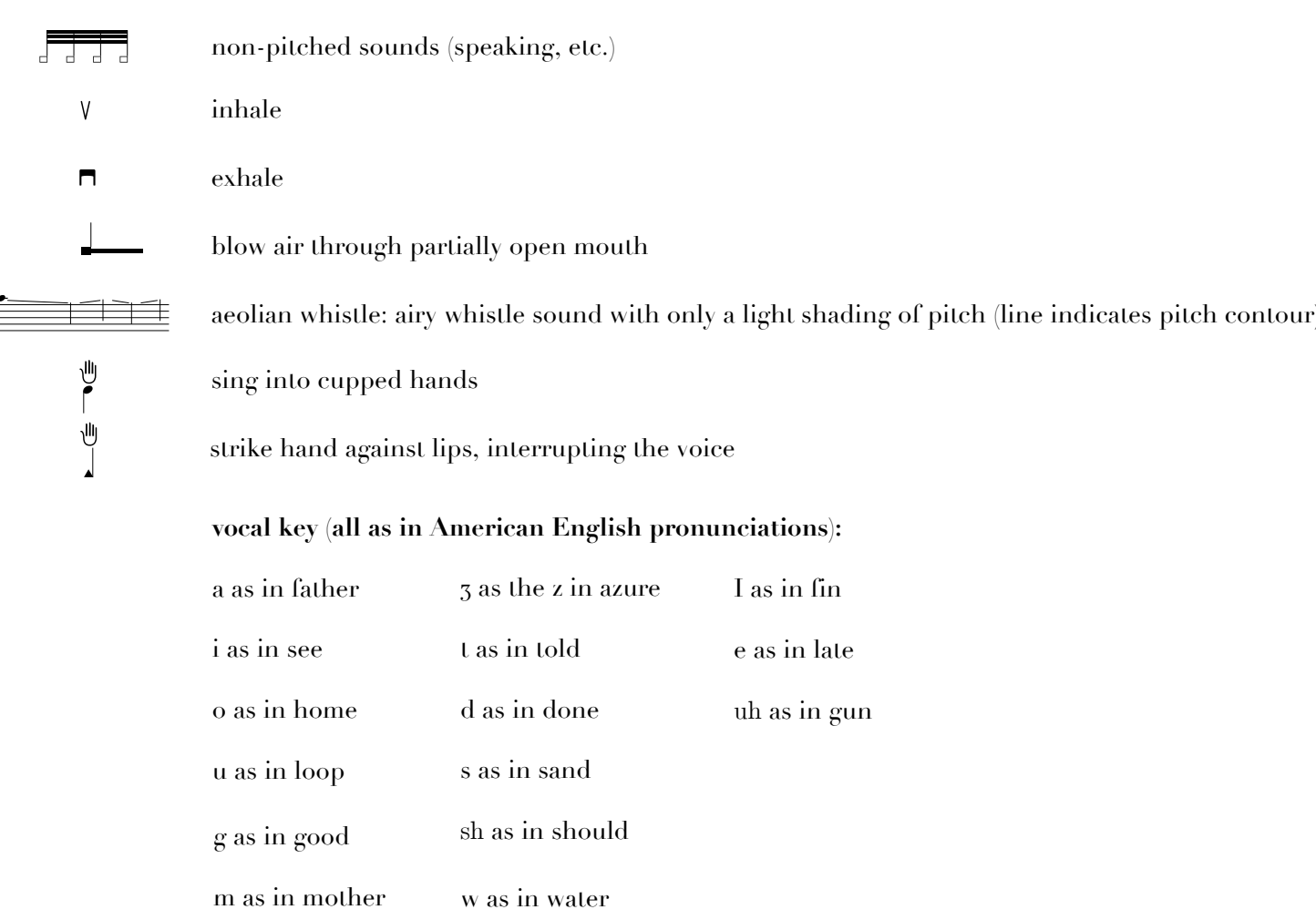

woodwinds

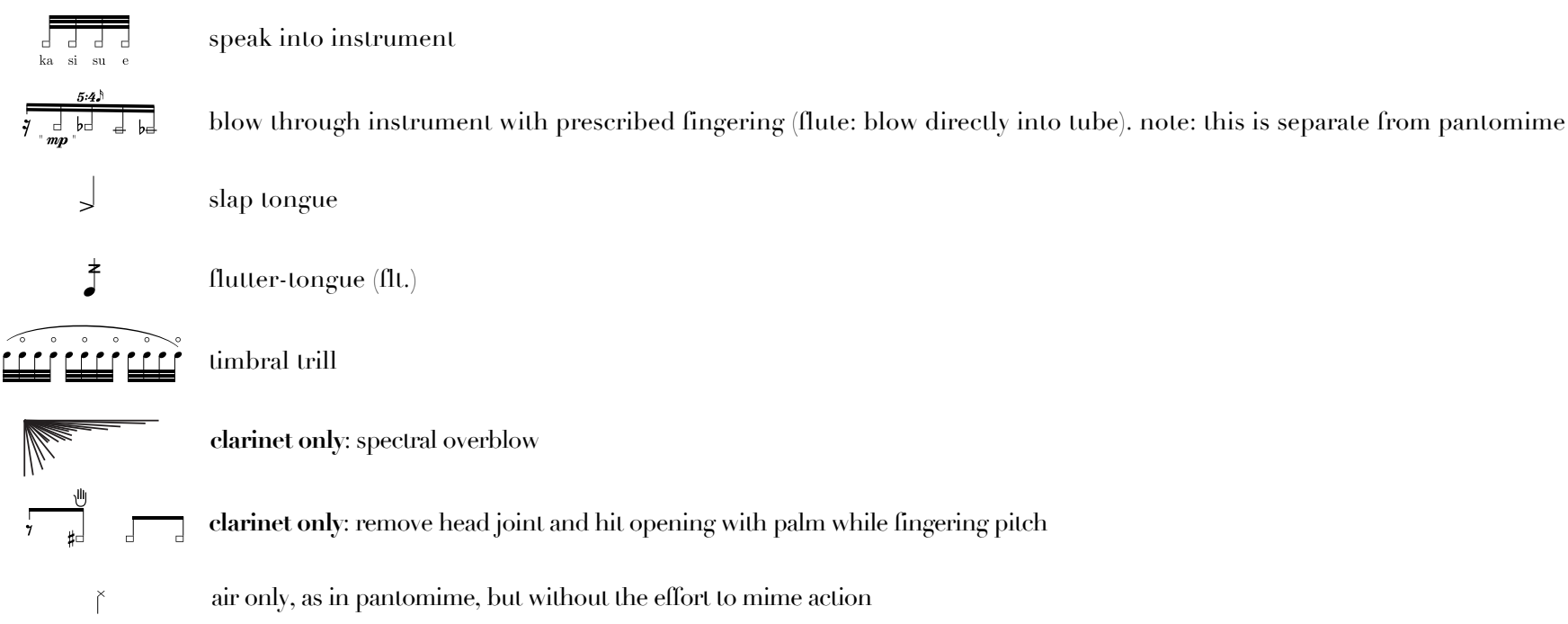

multiphonic fingerings (concert pitch)
b-flat clarinet:
bass clarinet:
苇!
alto saxophone:
$\vdots$
$\vdots$ 
brass

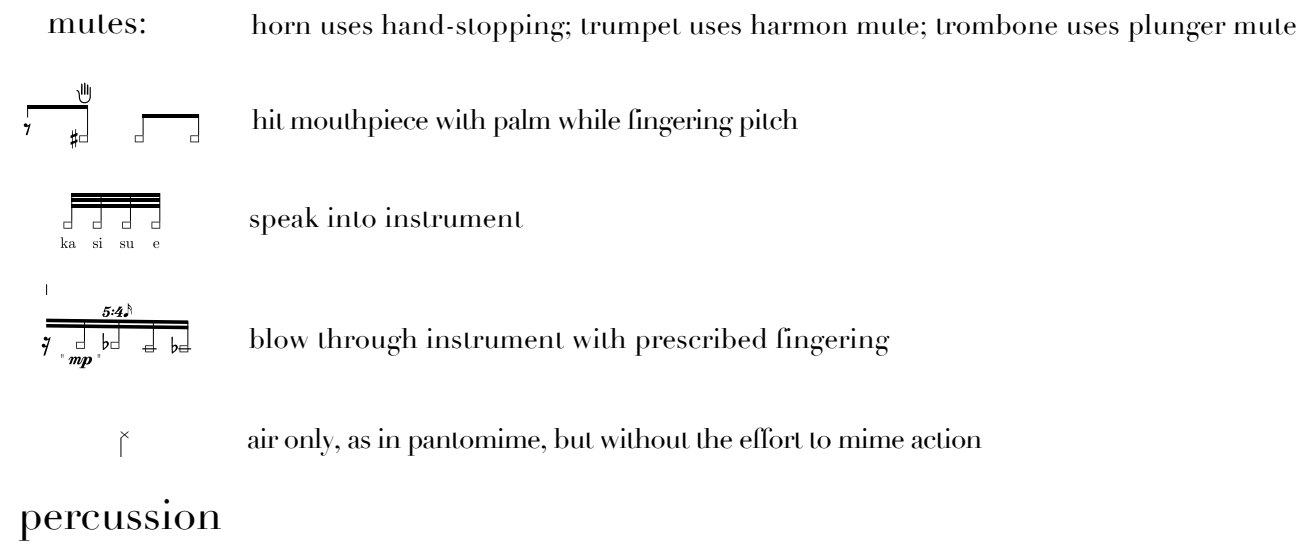

tam-tam on timpani

tam-tam should be placed on the timpani face-down; the range of the timpani is represented by a two line staff, the top line representing the highest pitch possible, and the bottom line representing the lower pitch possible

piano

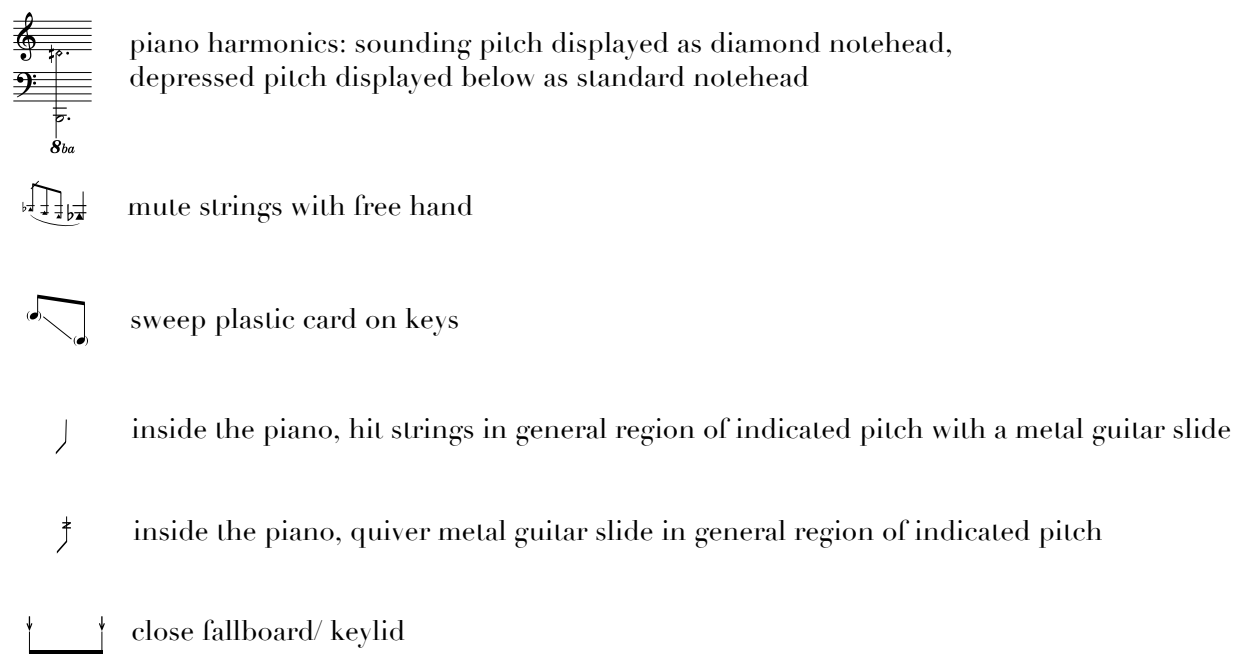


it is preferable that the player use a guitar that is capable of decent sustain (optimally a solid-body, through-neck instrument) along with a tube amplifier when possible for reference, the composer used a Fender Jazzmaster and a Fender Blues Deluxe 40-watt amplifier.

player will need:

- volume pedal for swells

- natural-sounding distortion (either as a pedal or onboard a high-quality amplifier)

- an EBow

r.m. rapidly and erratically sweep left hand over medium range of neck without plucking

夷 slide left hand without plucking in general area of indicated pitches

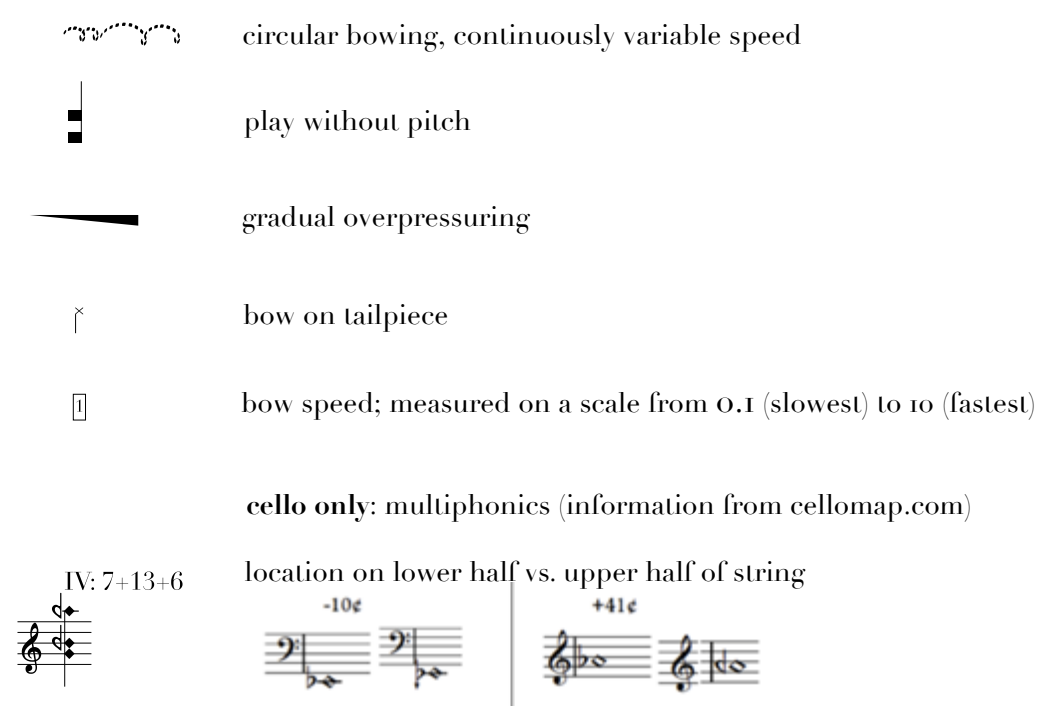

all players should be lightly amplified, with very little compression, and mixed so that the amplification balance is even. the percussionists should each have at least three microphones running throughout their setup. 
i.
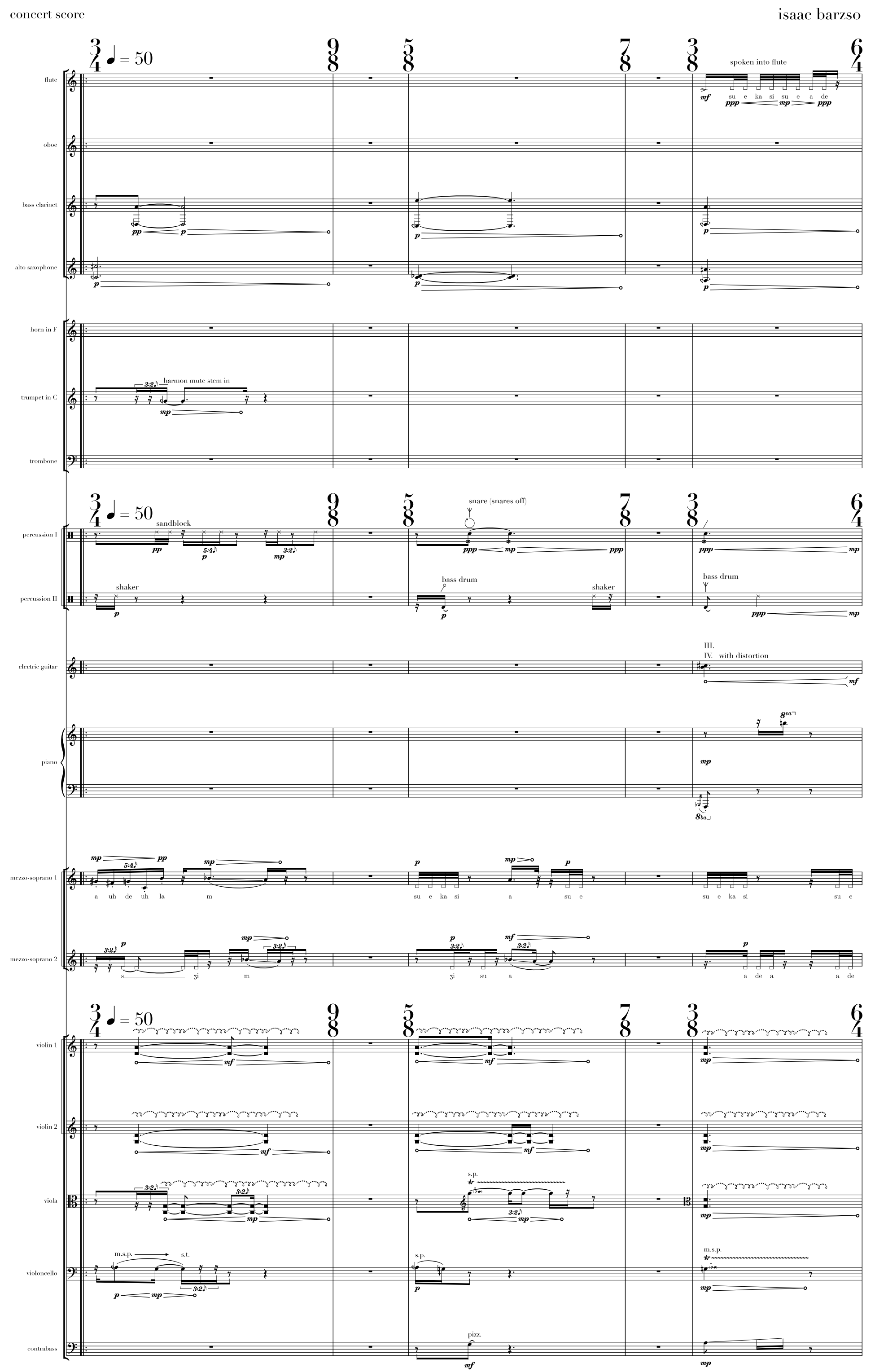

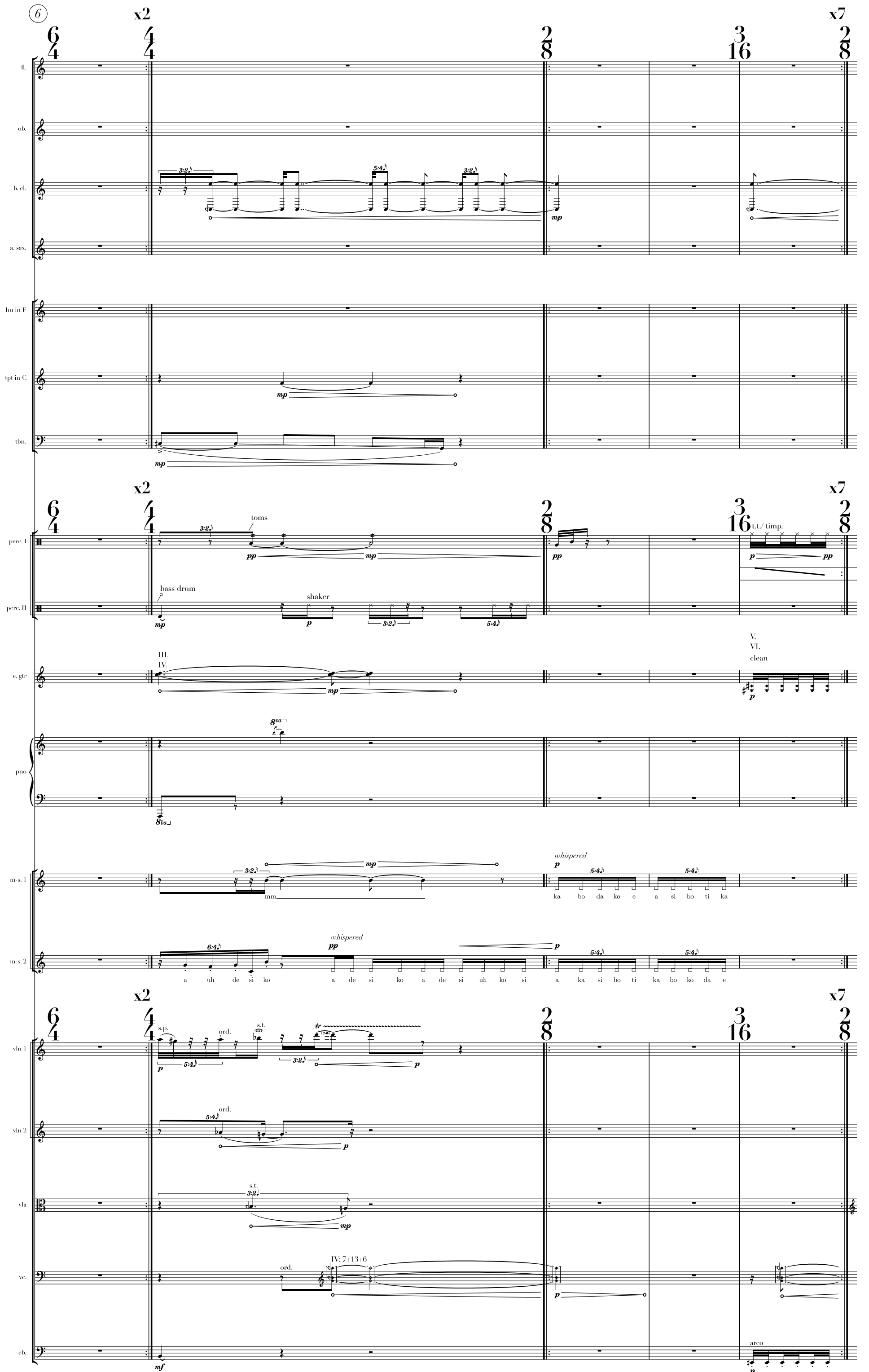

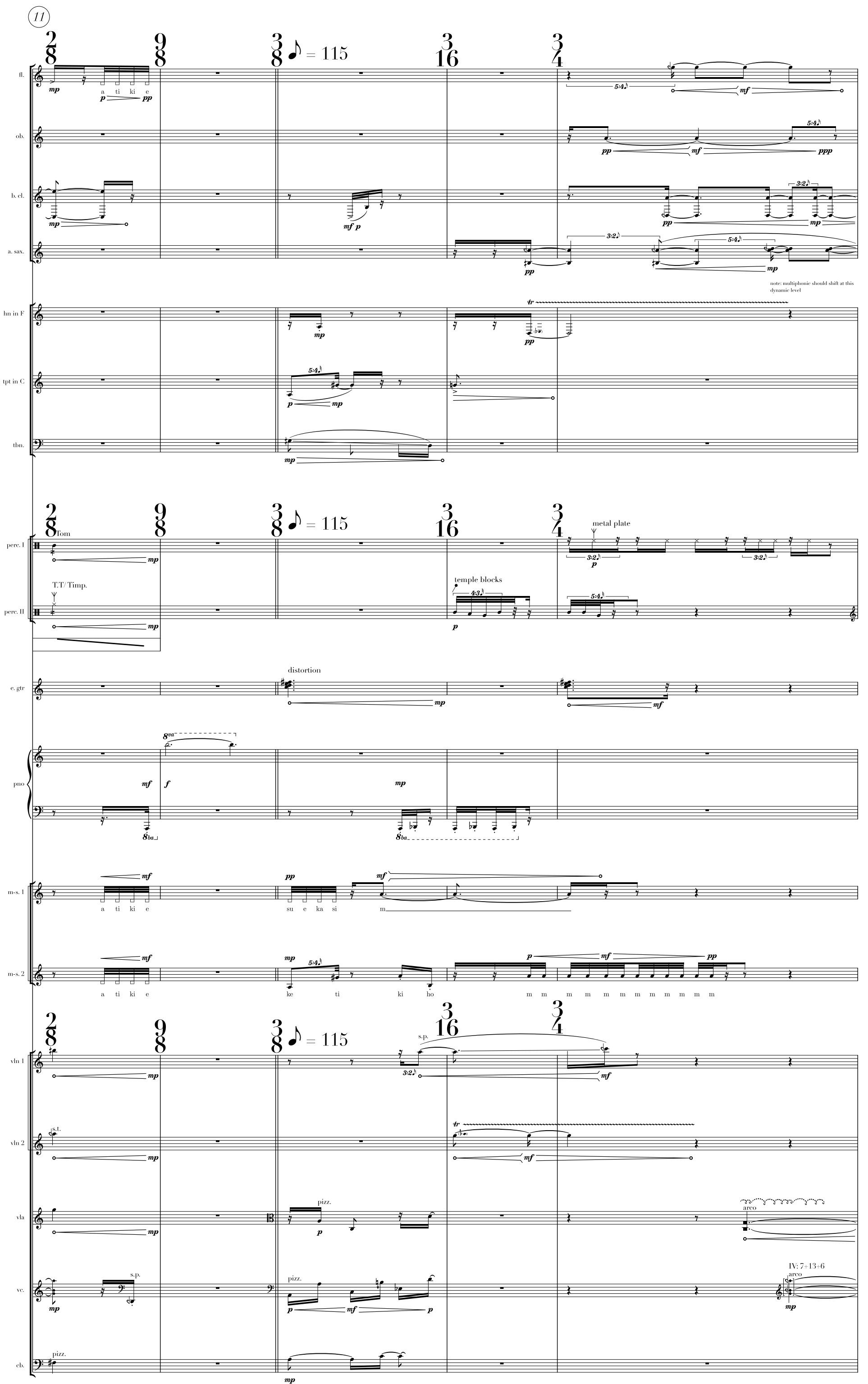

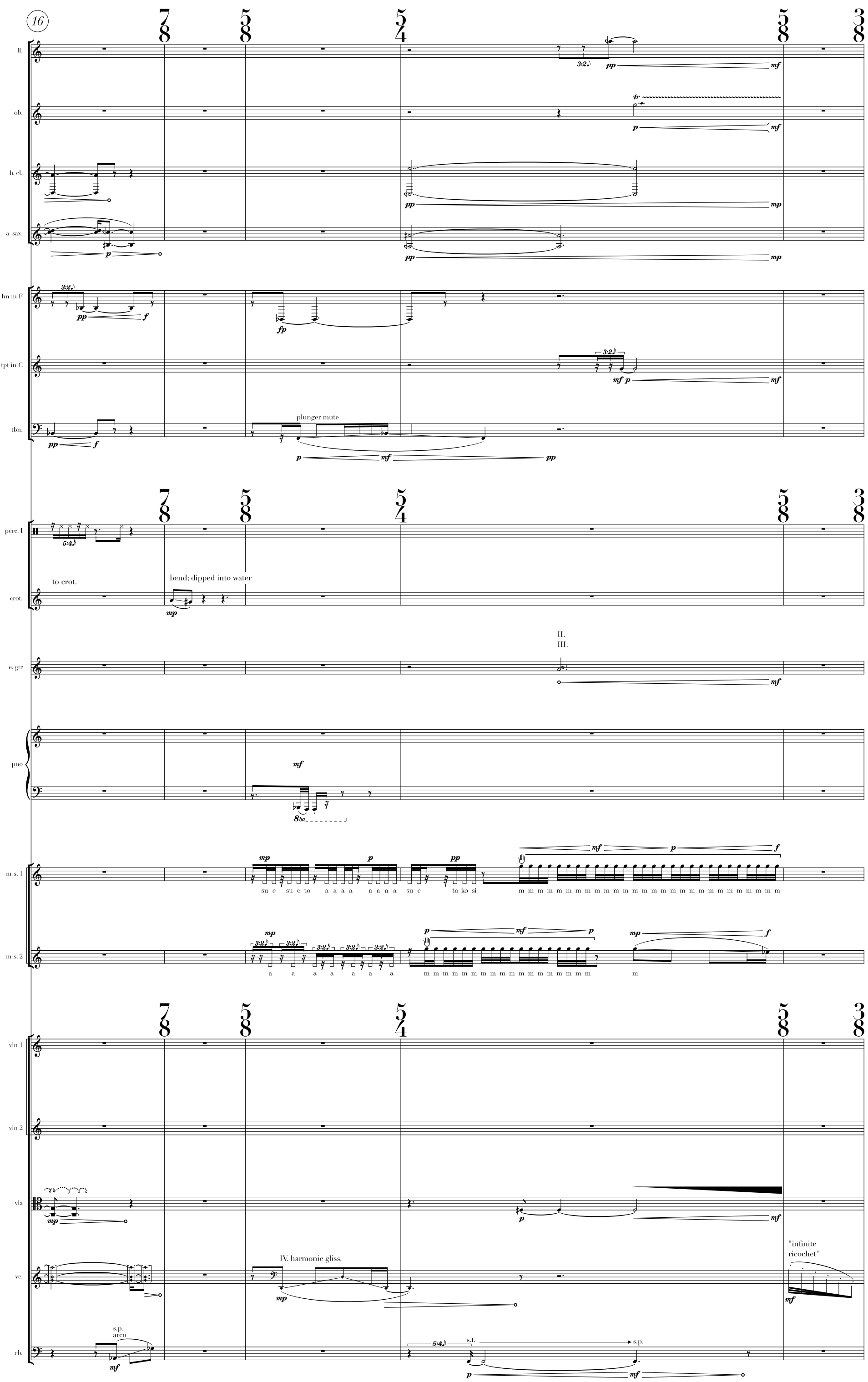

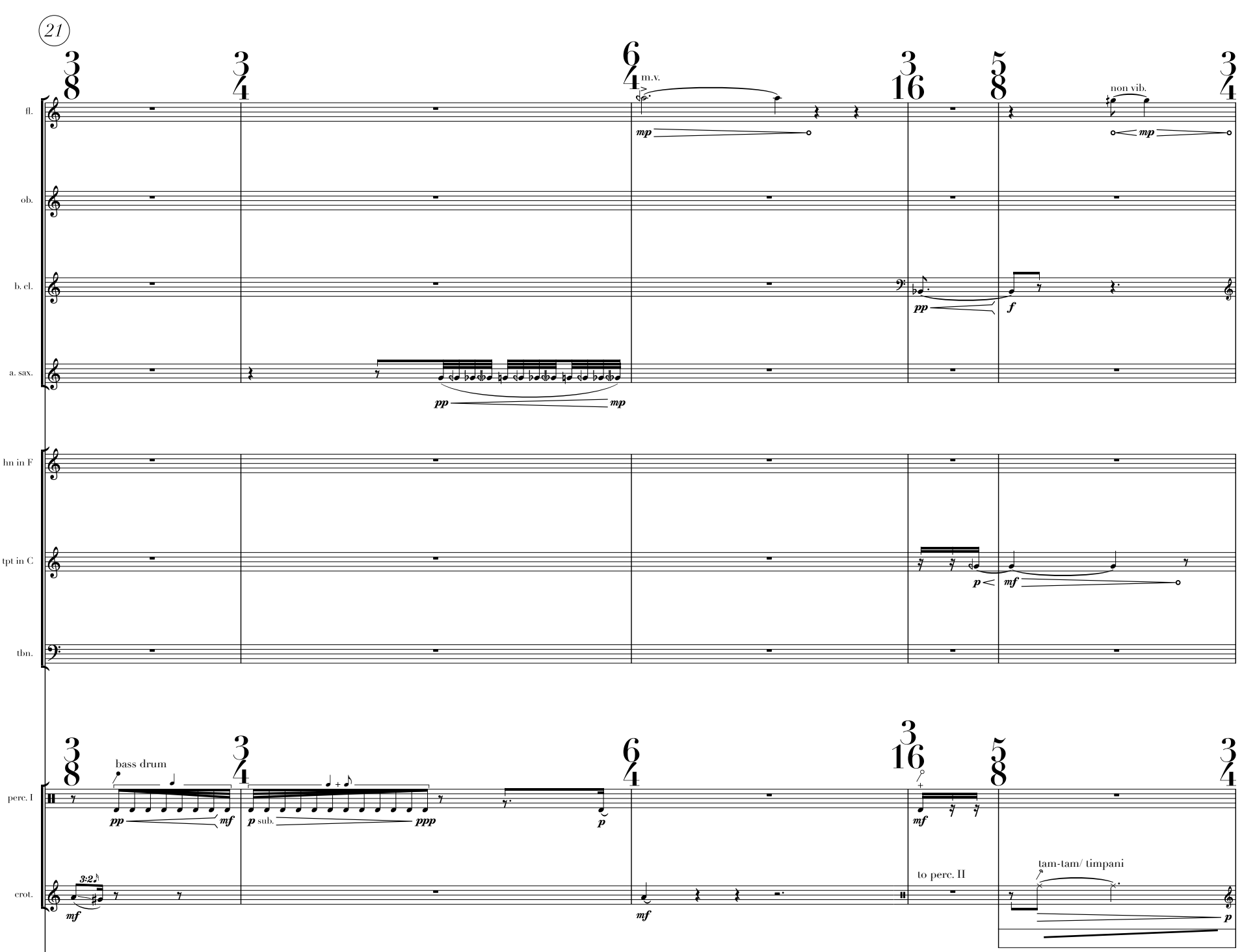

wit FBow
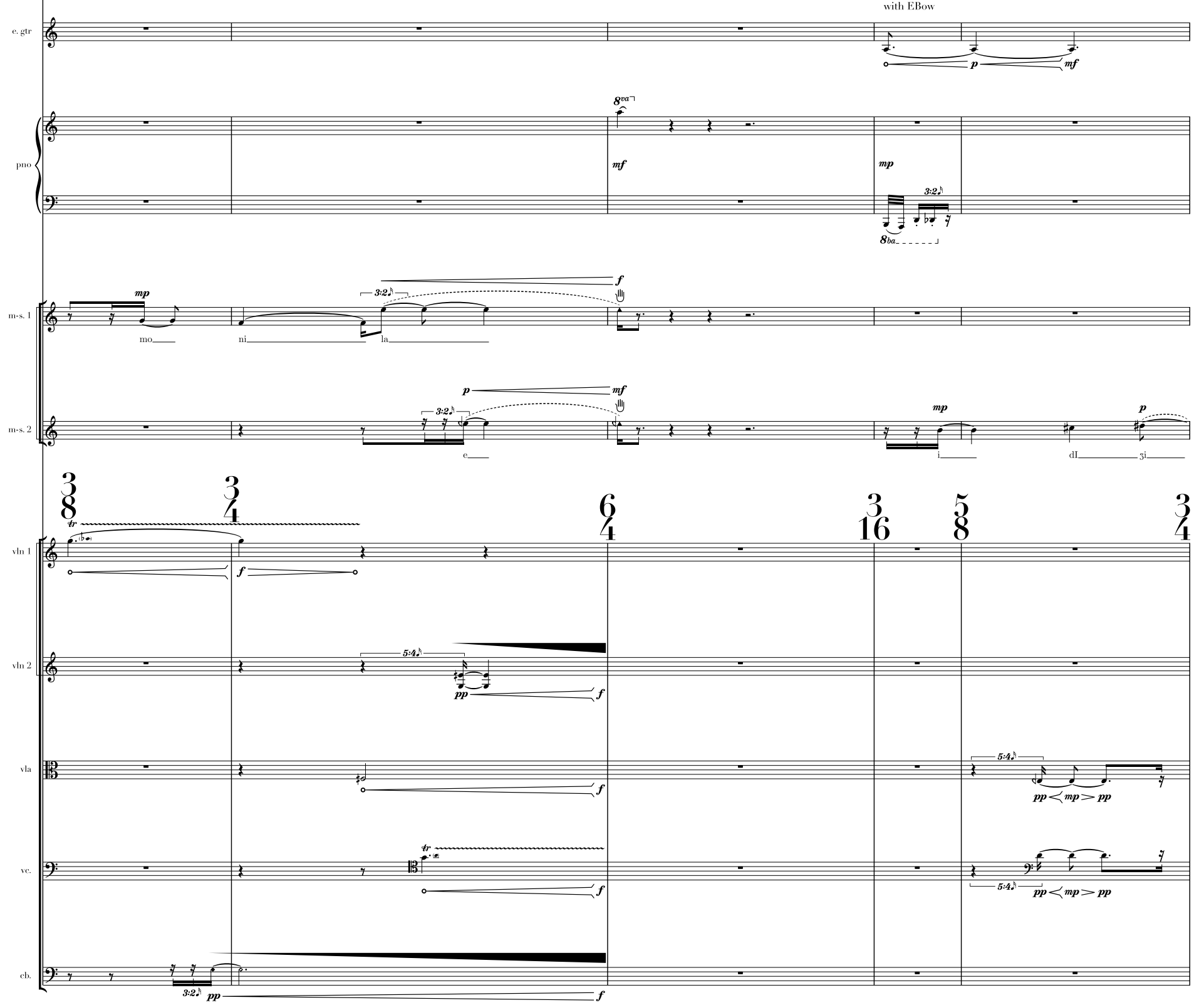
6
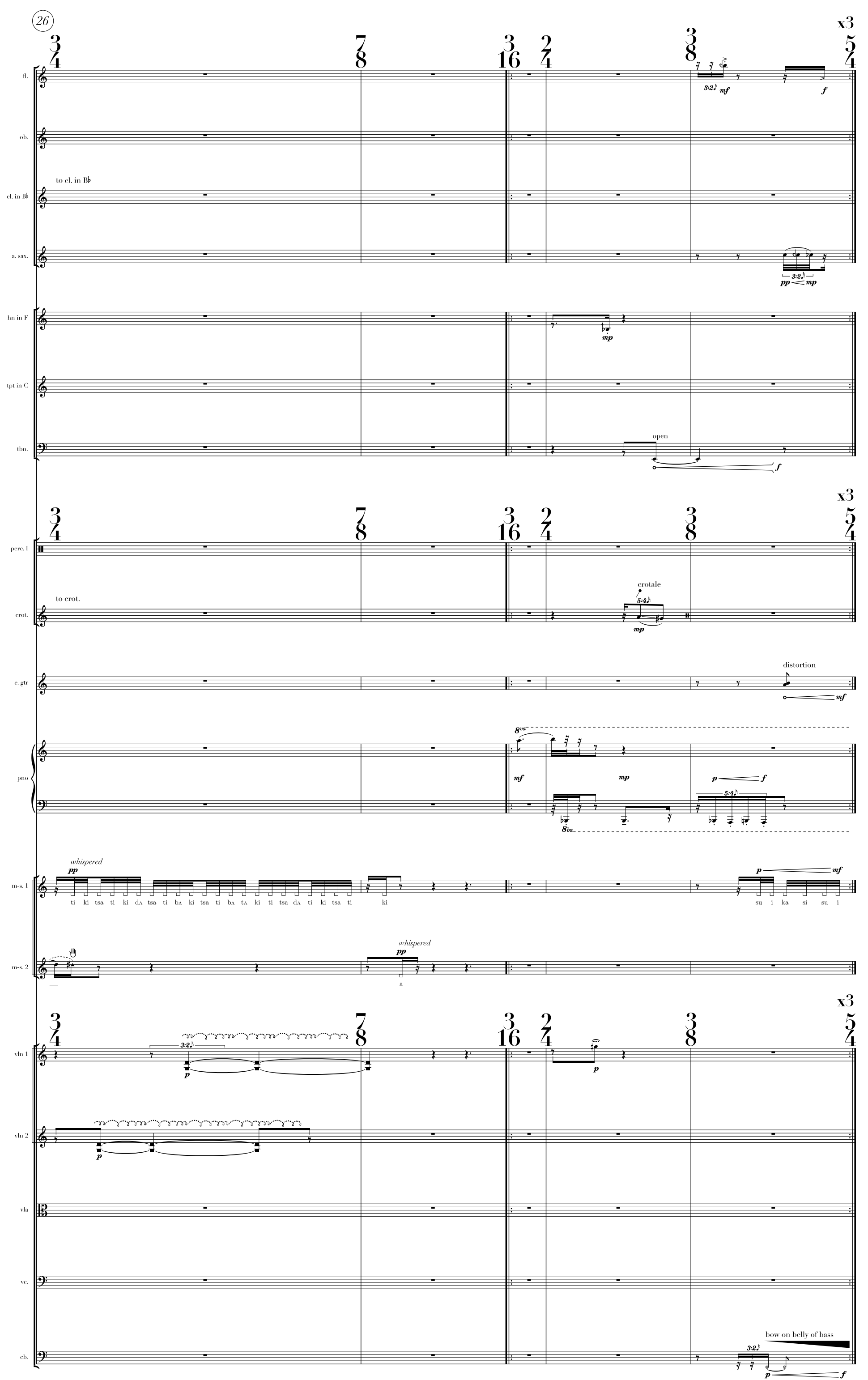

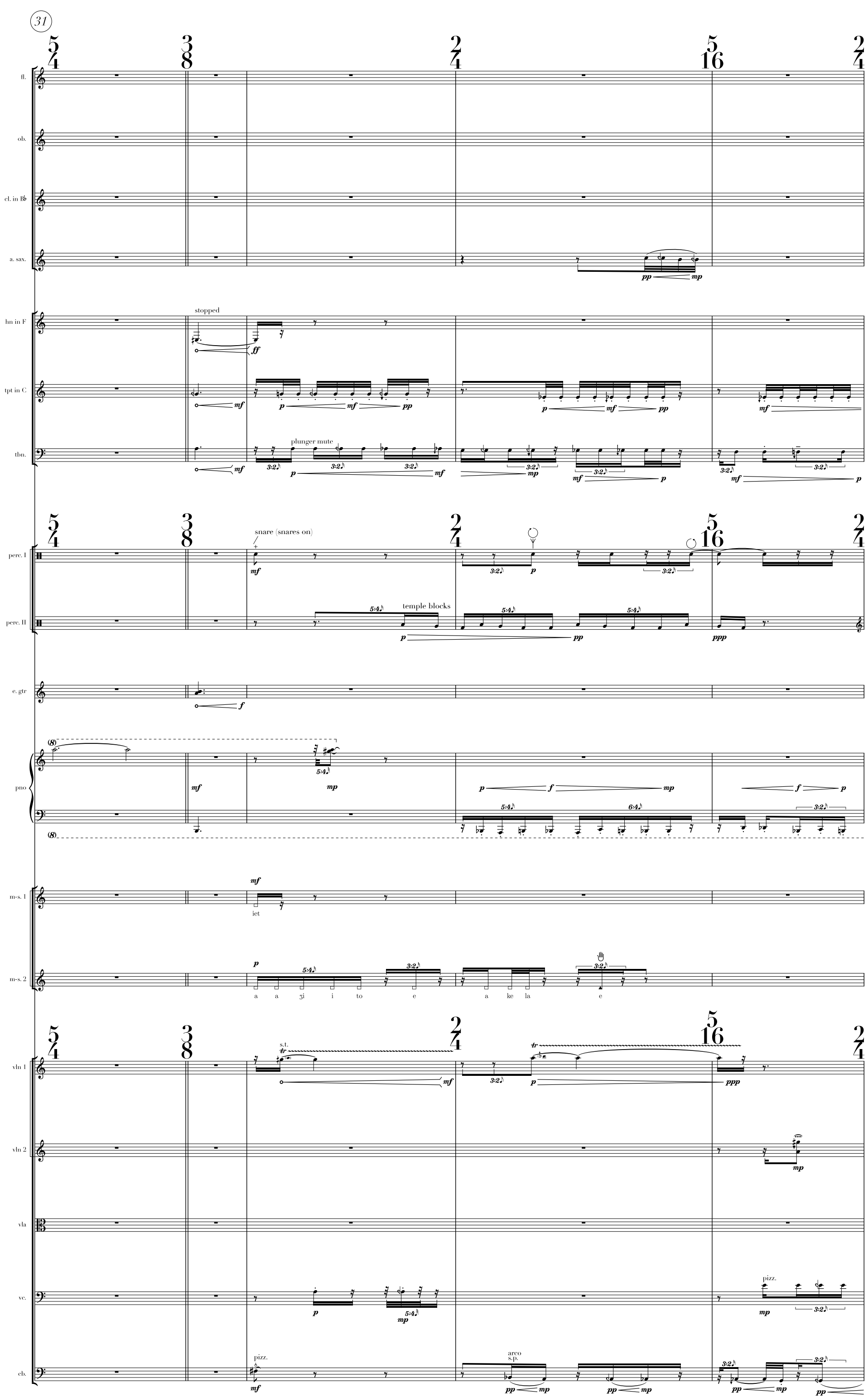

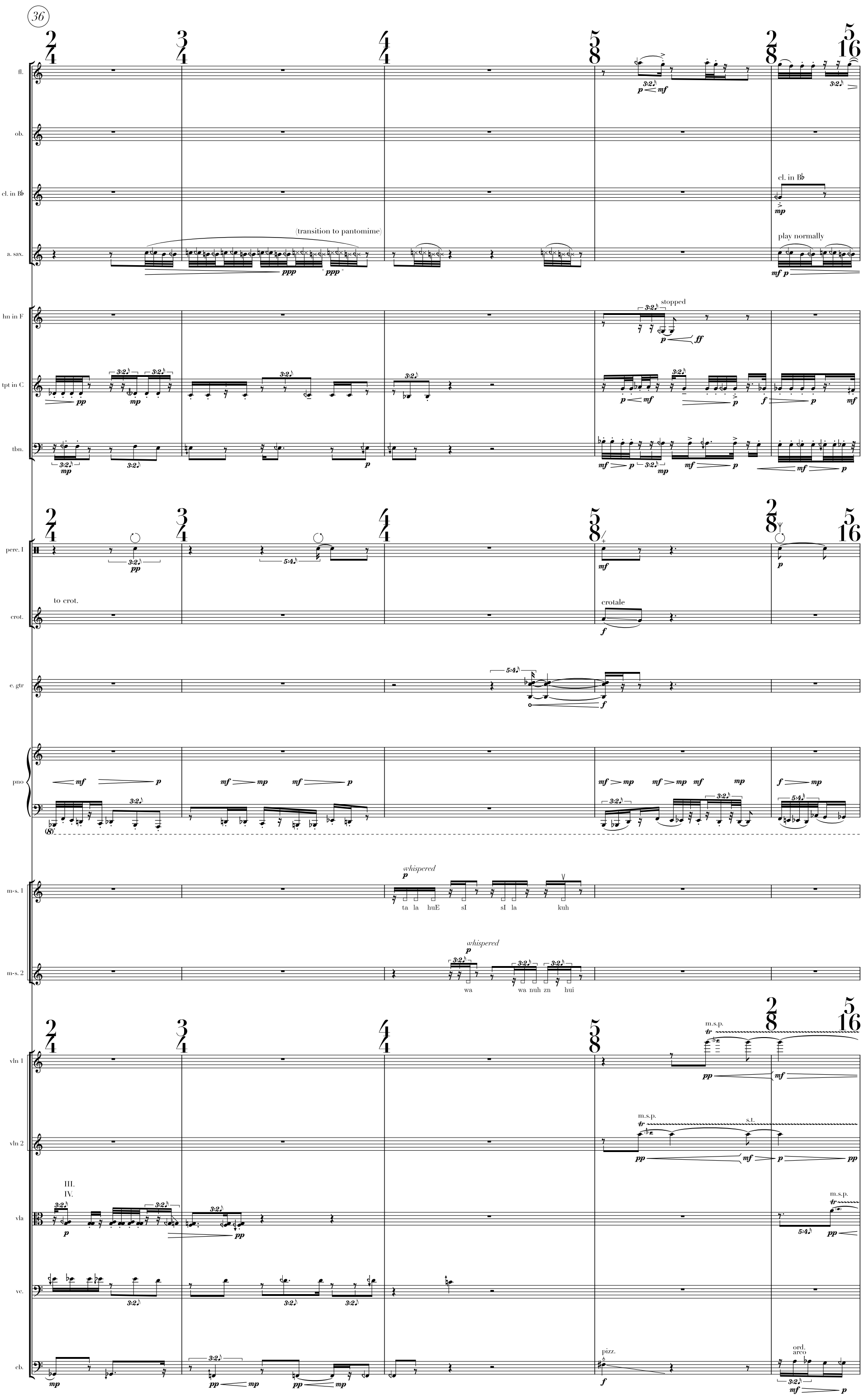

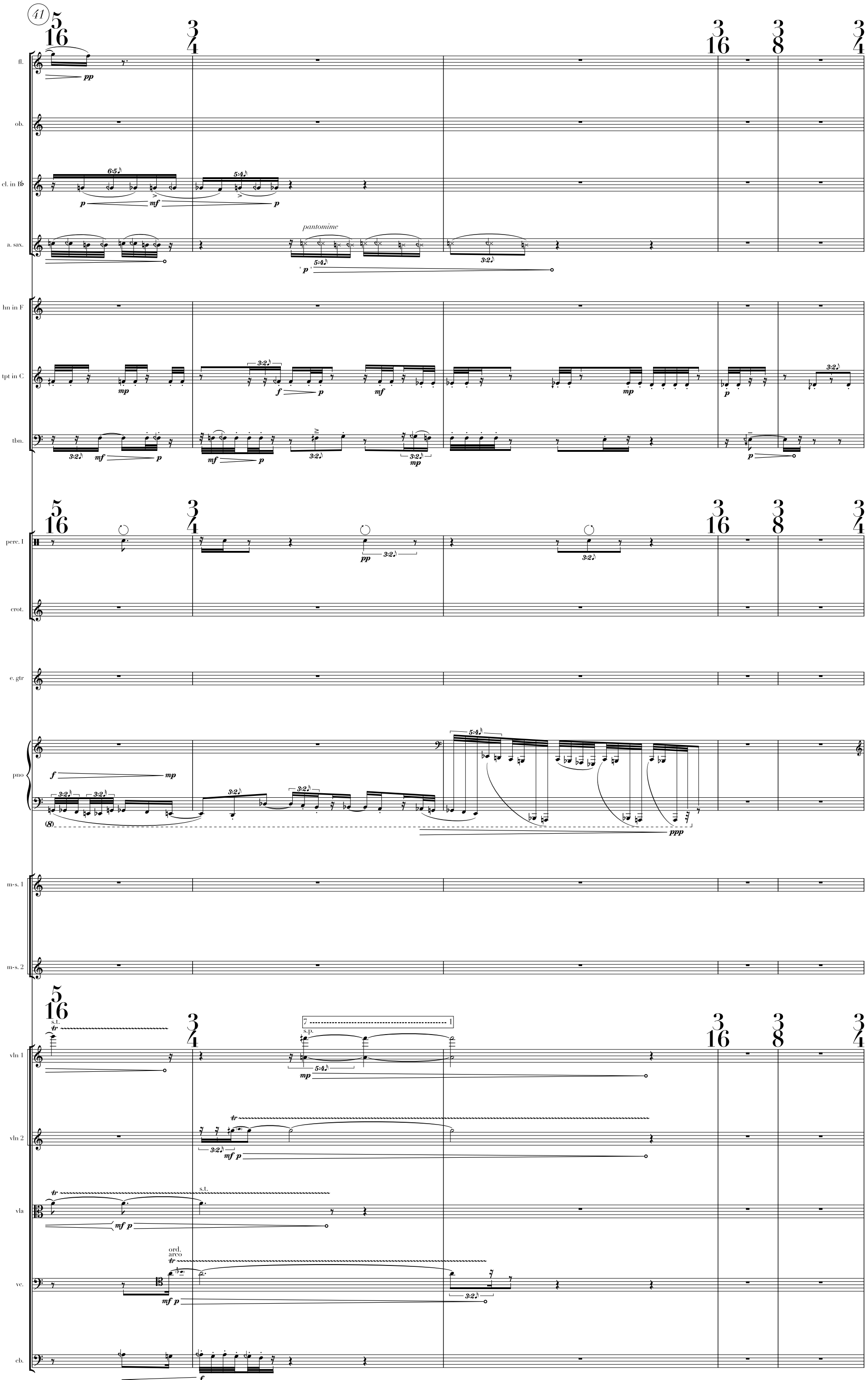
10
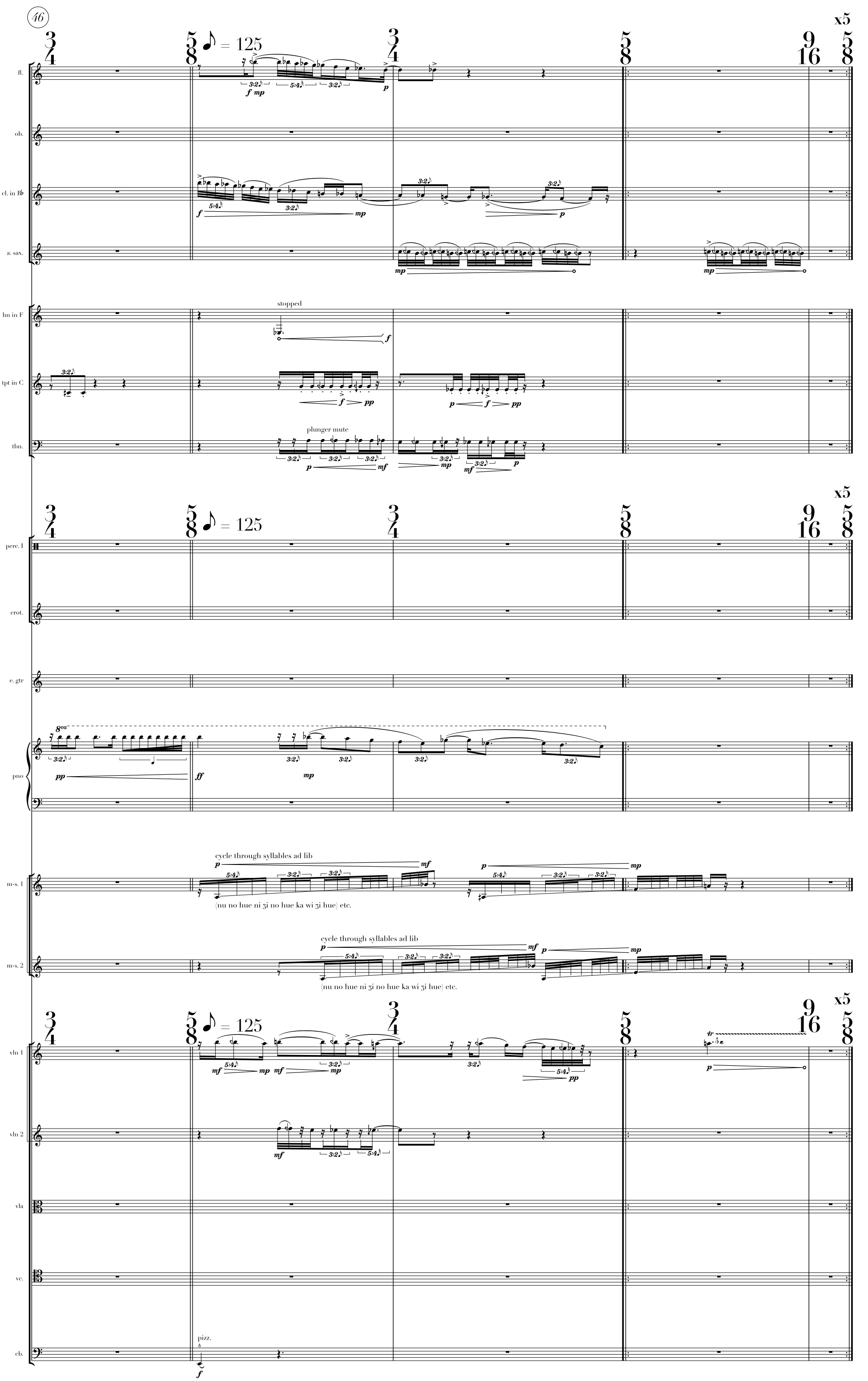


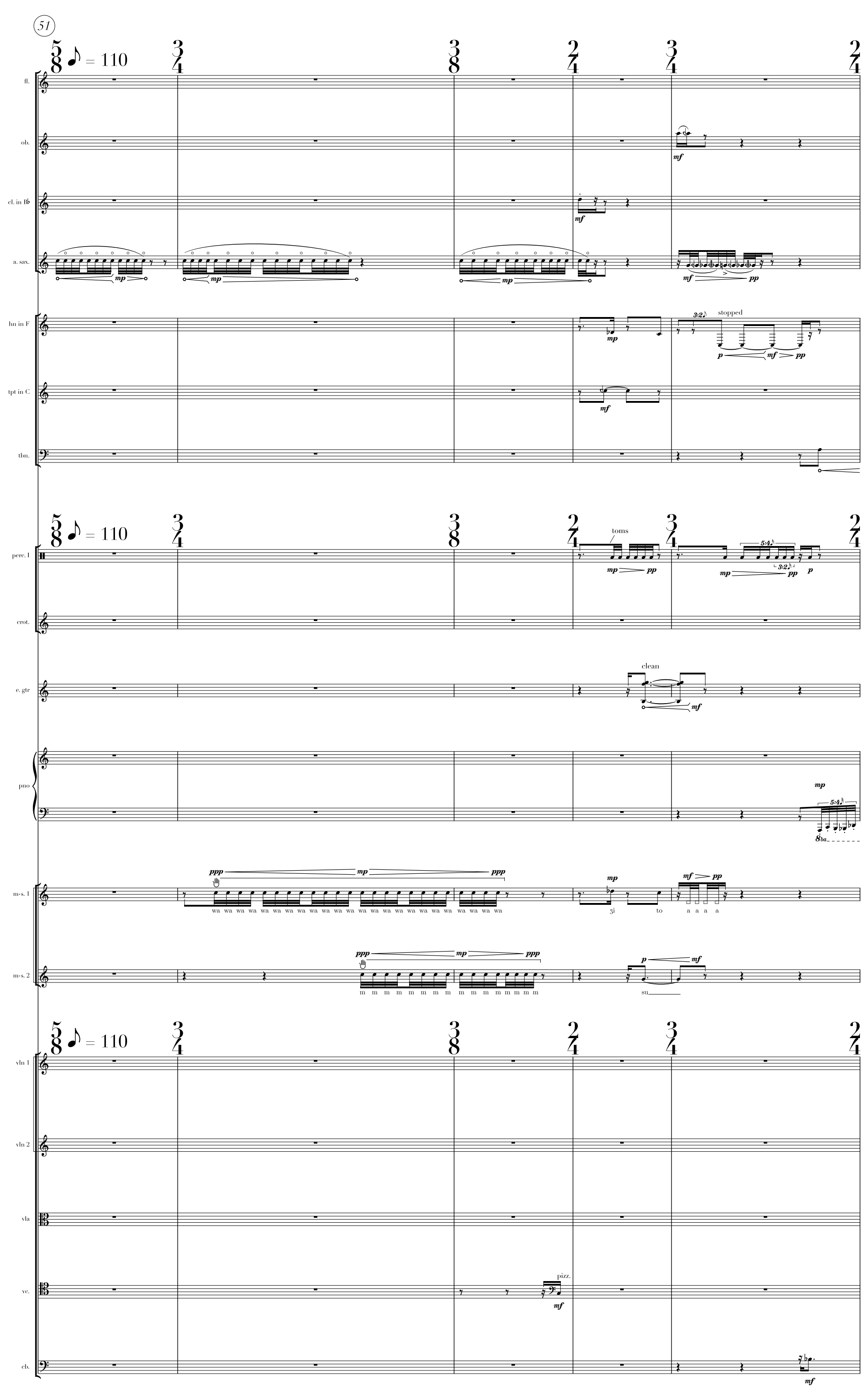


12
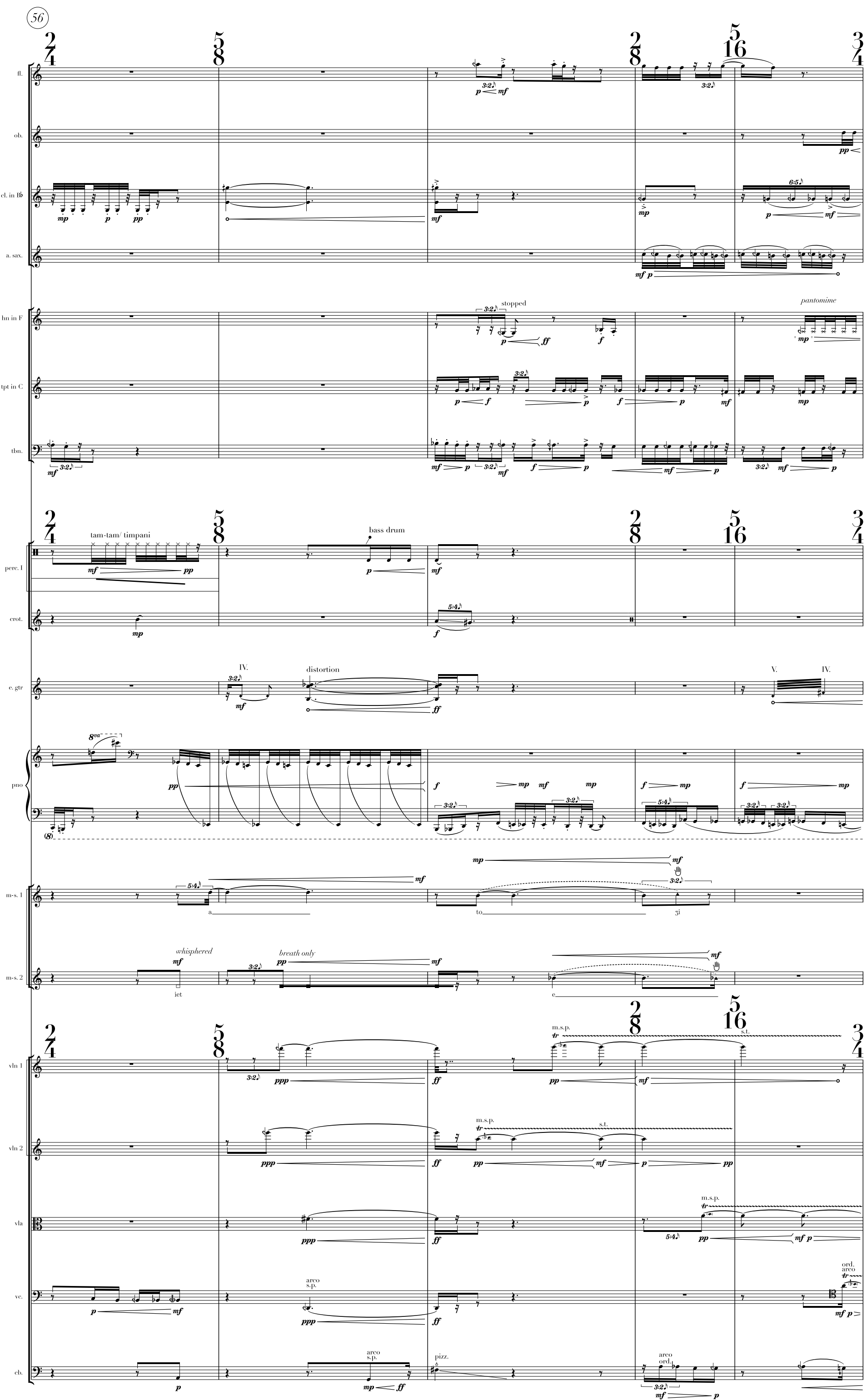

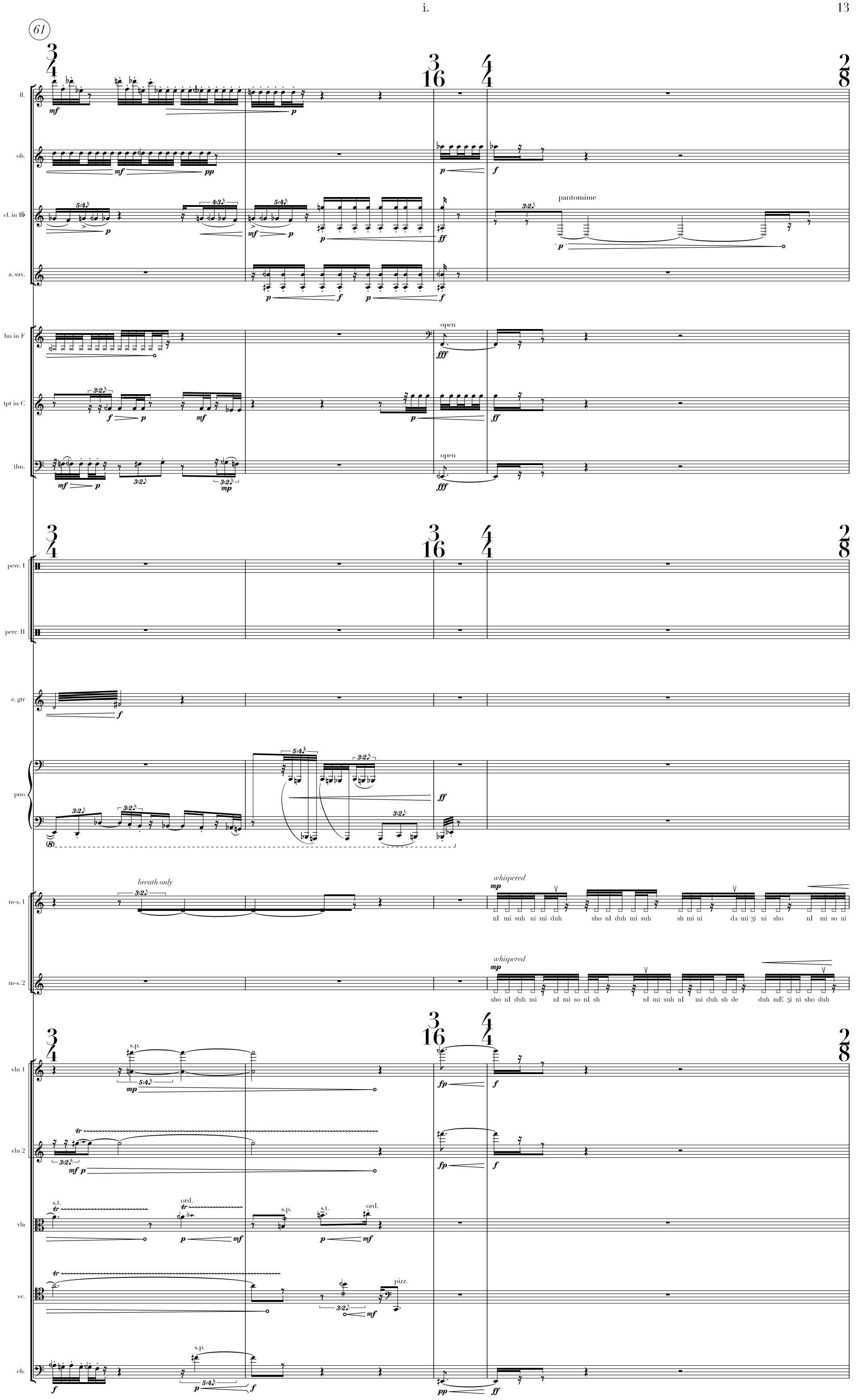
14
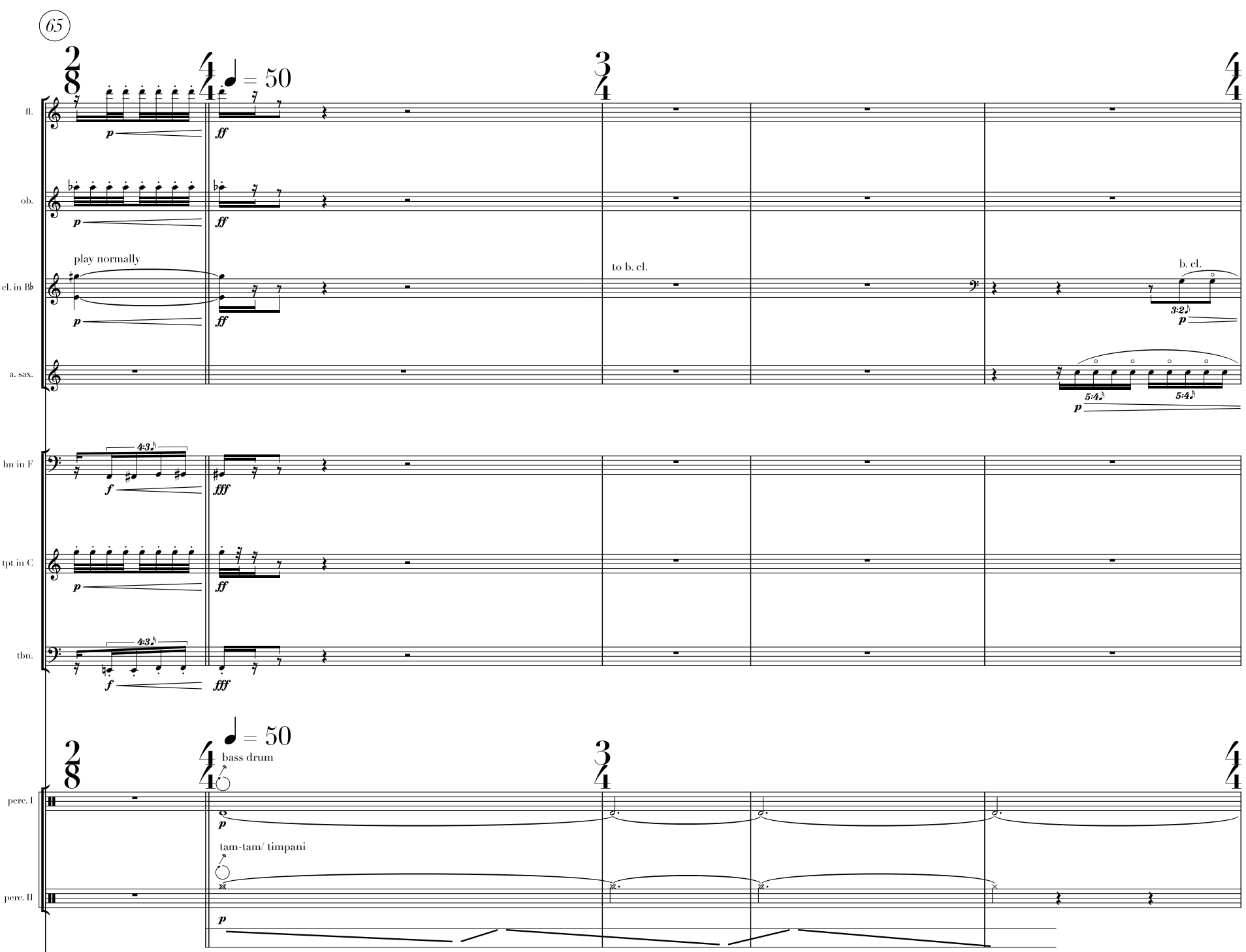

of

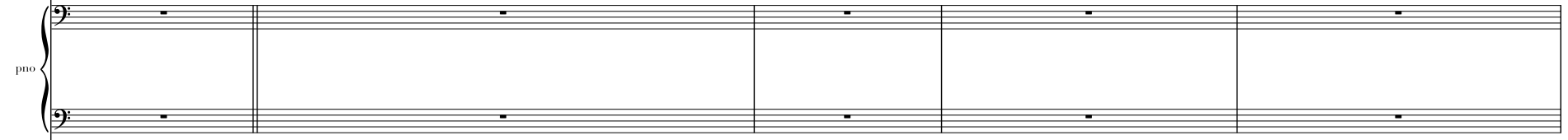

(a)

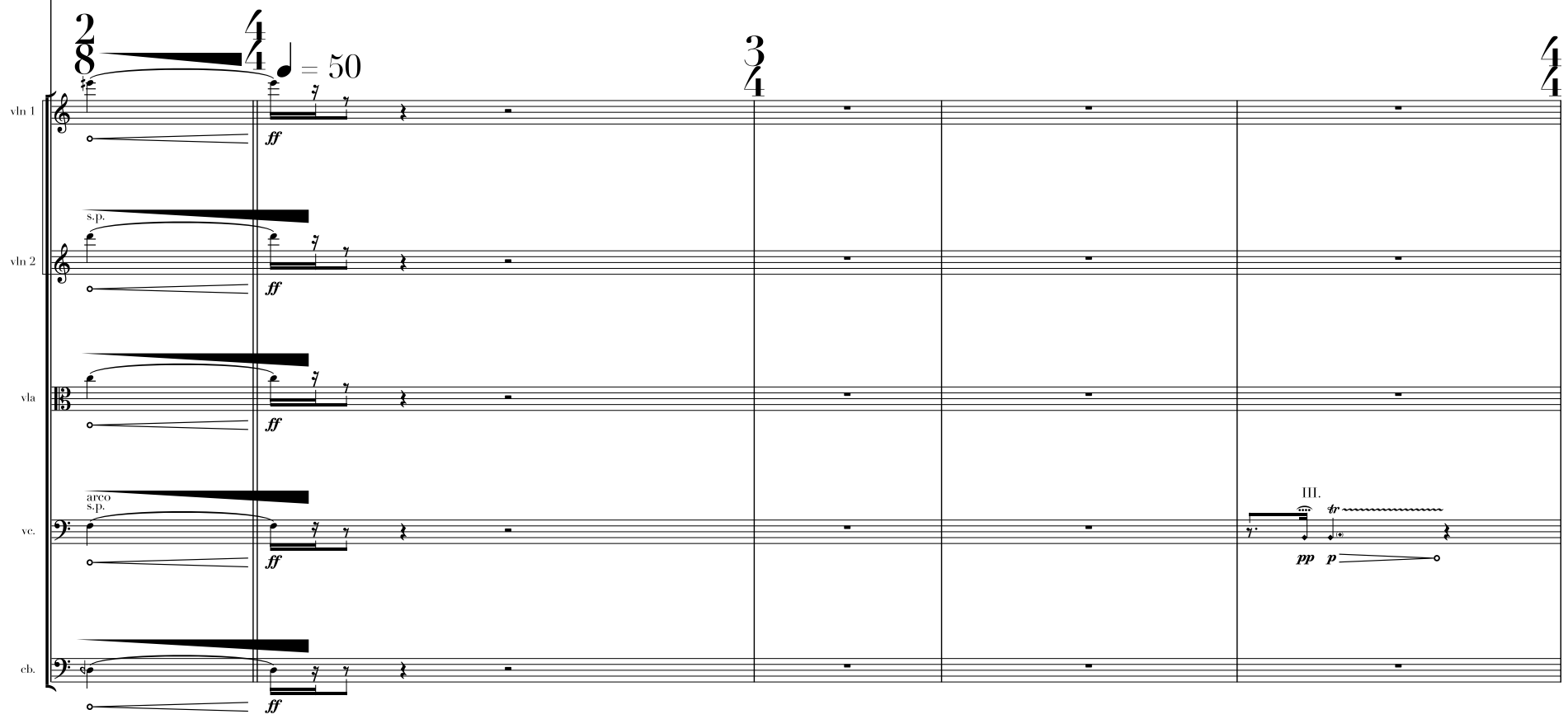



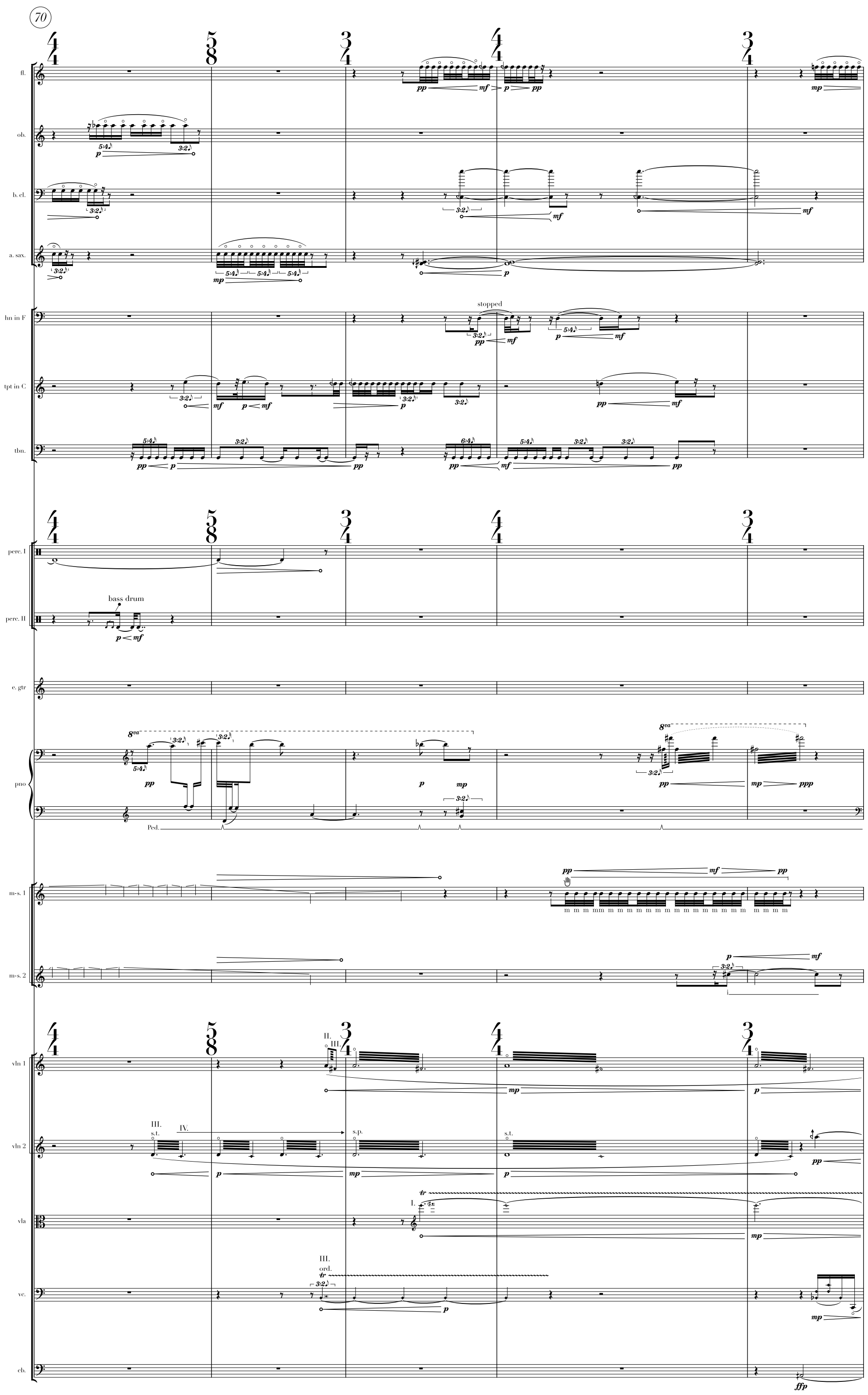

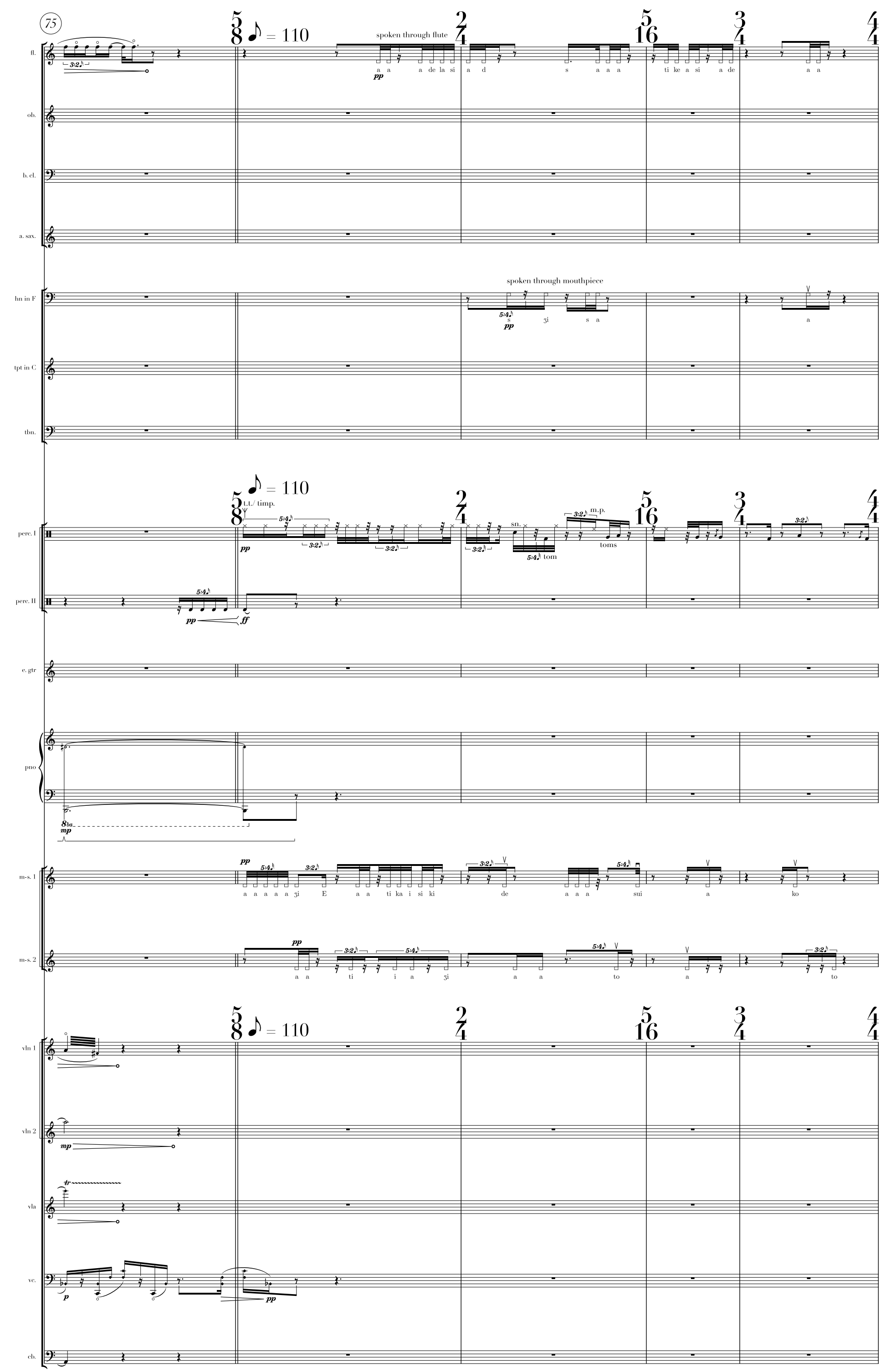
i.

17

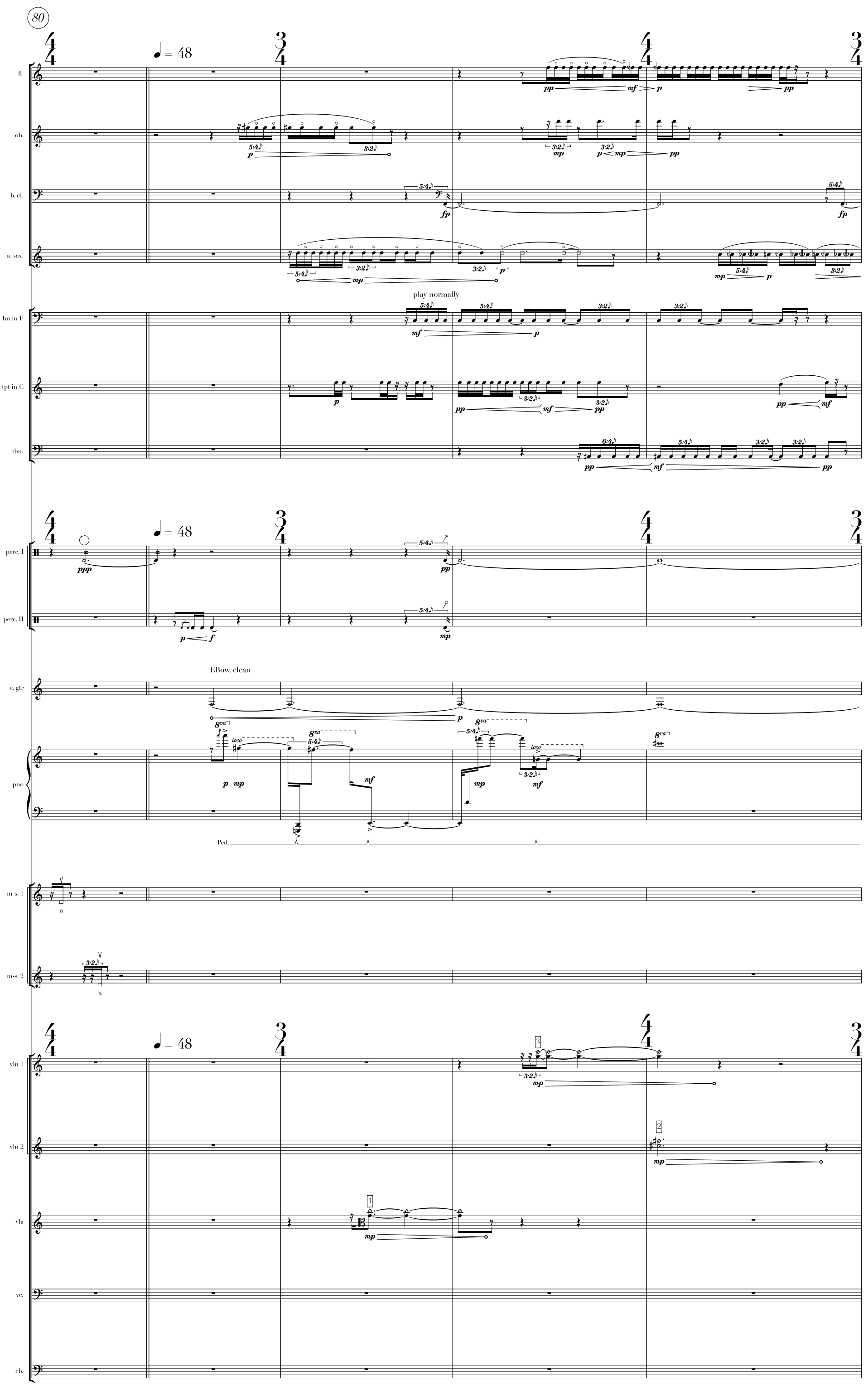



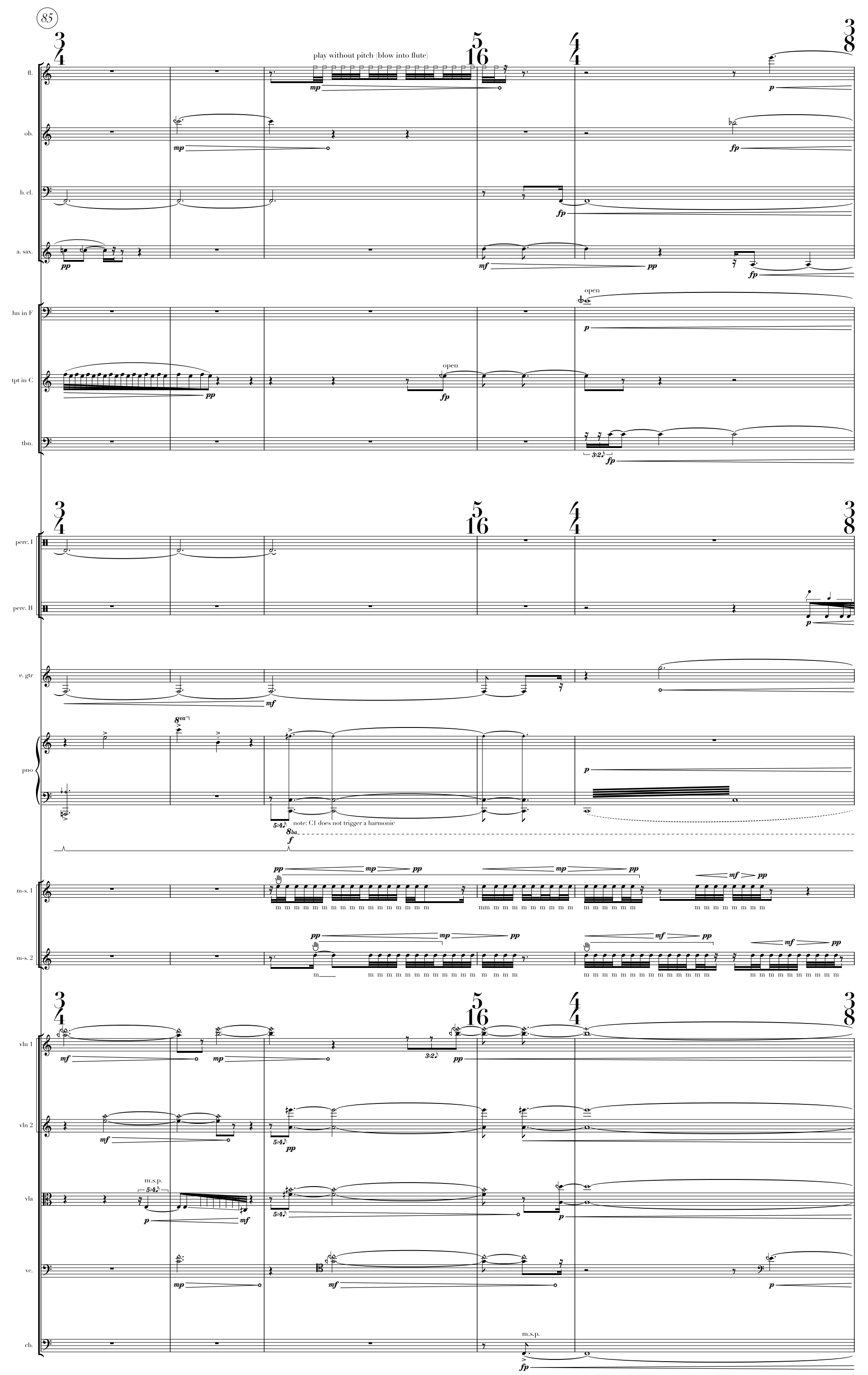

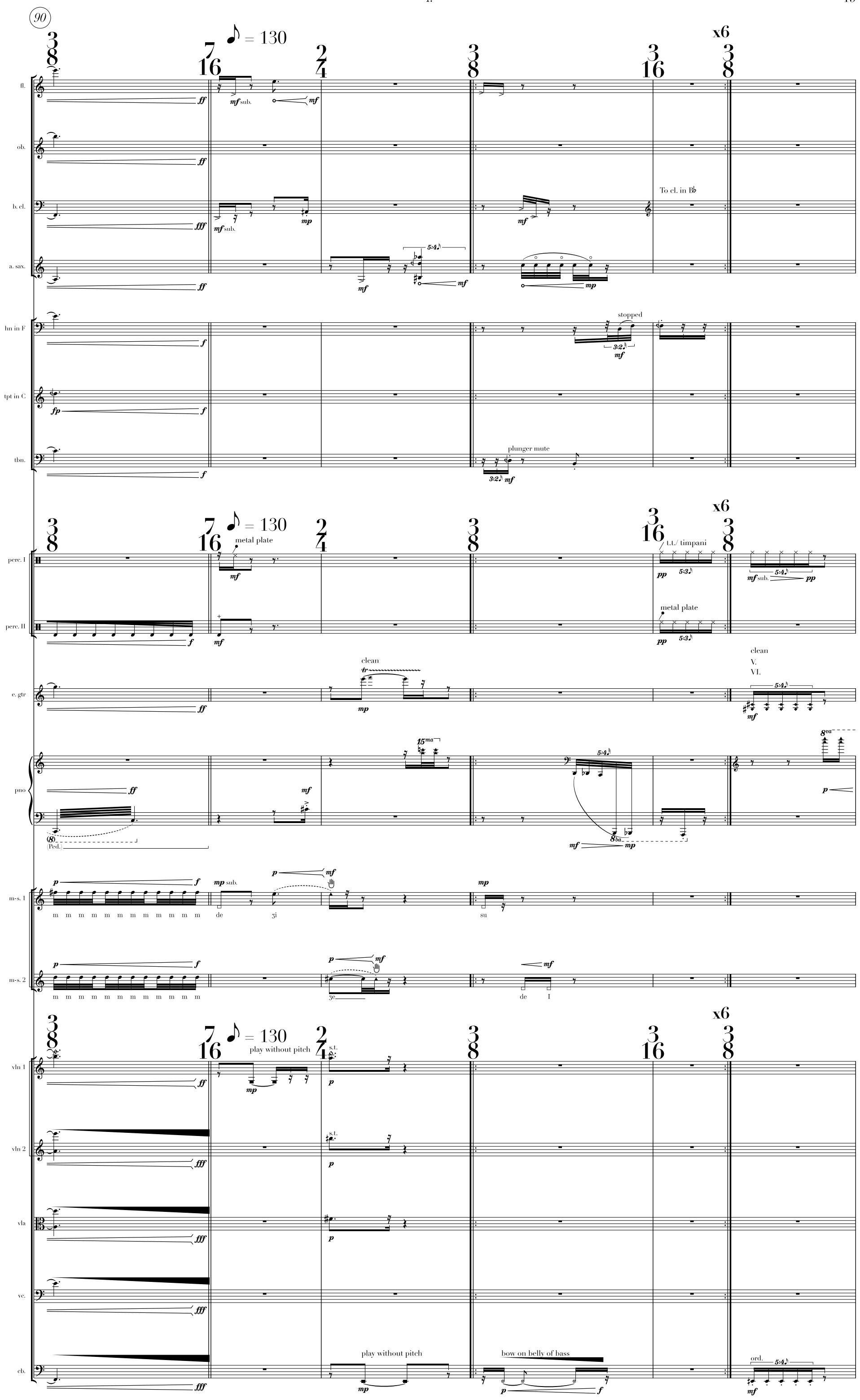

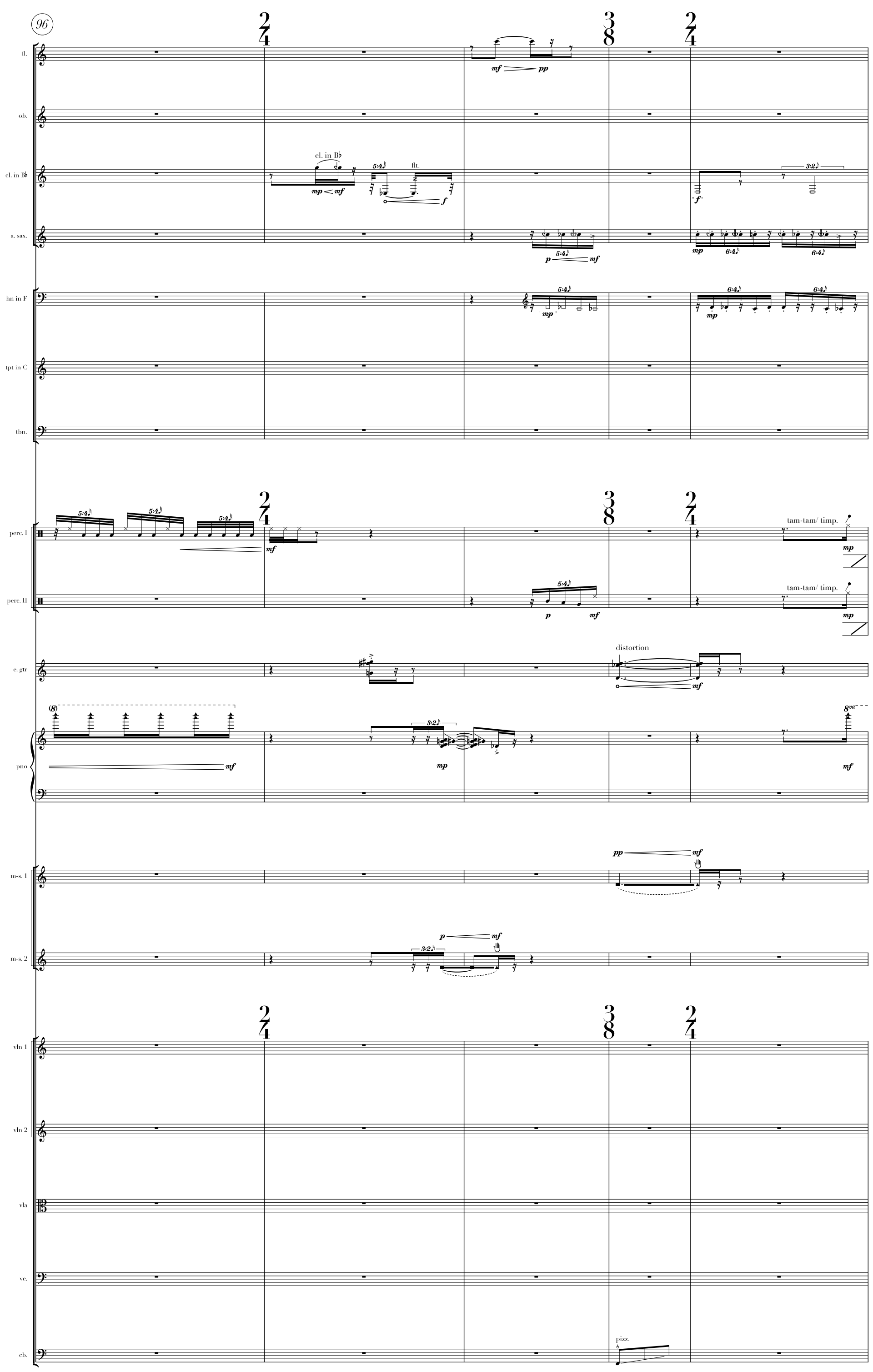

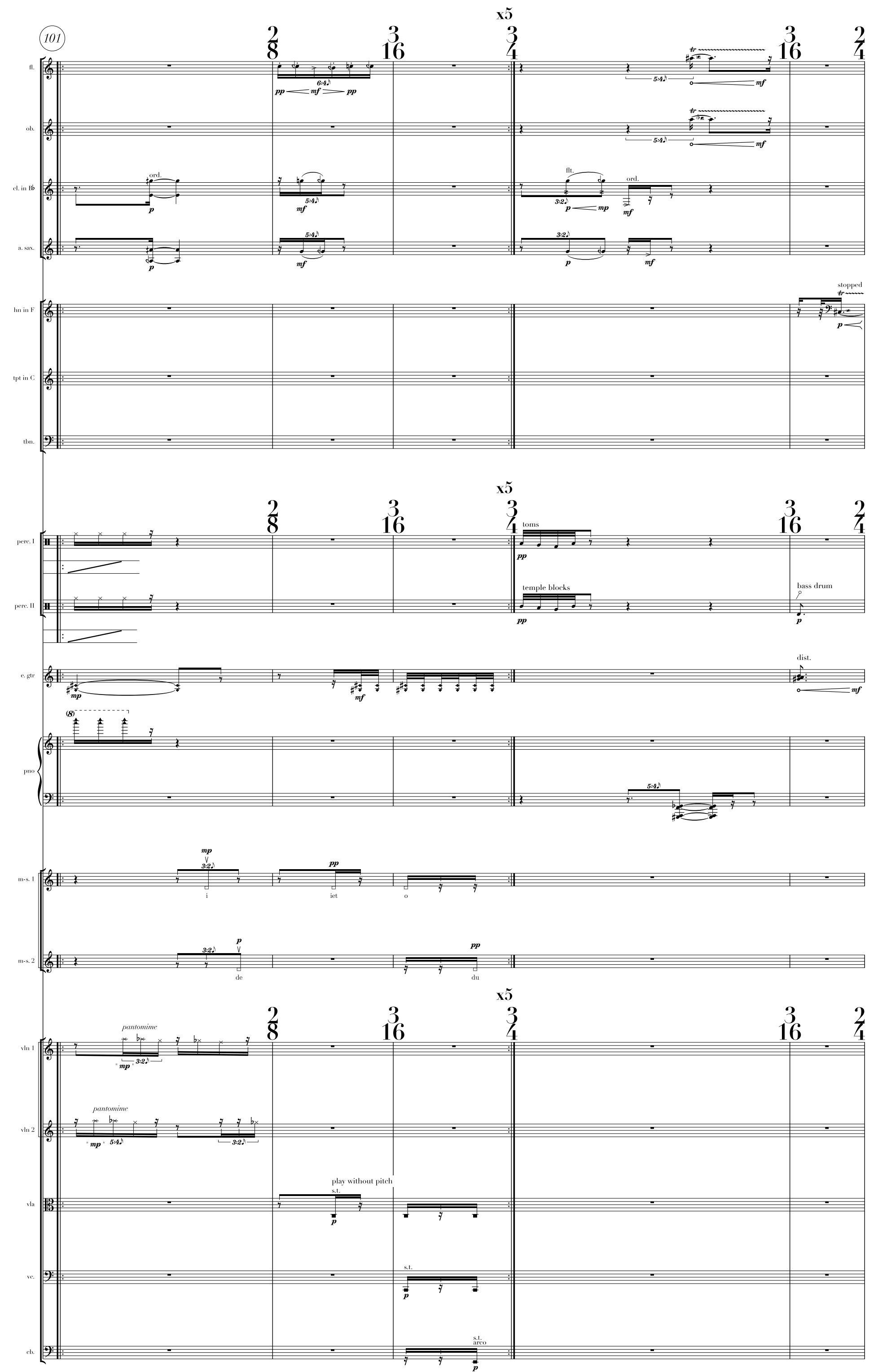
22

i.
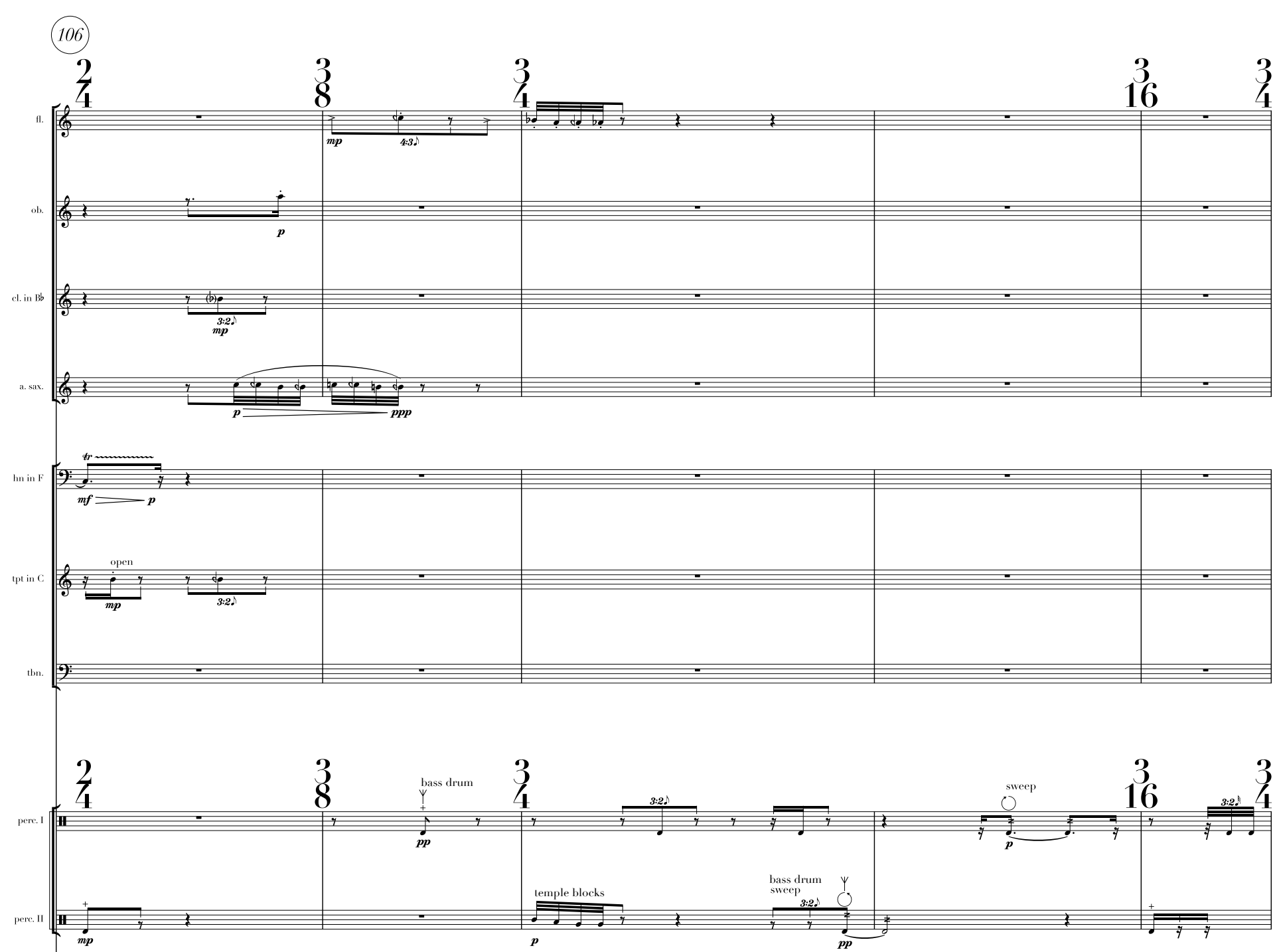

e. gr
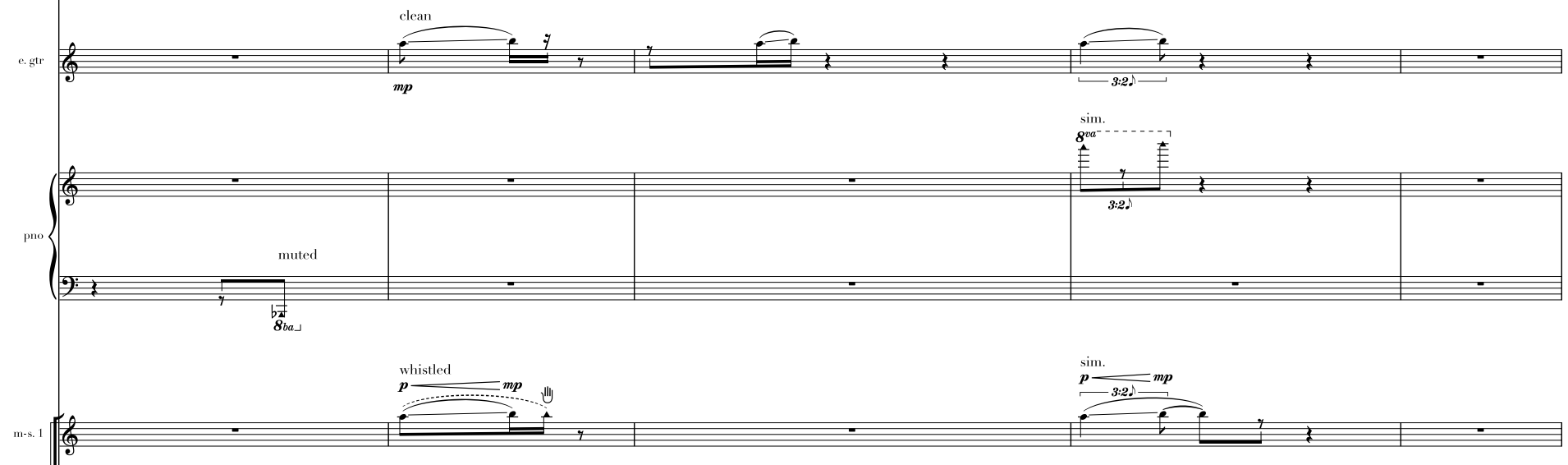

sim.

whistled

$\frac{2}{4}$

3

3

36

3 

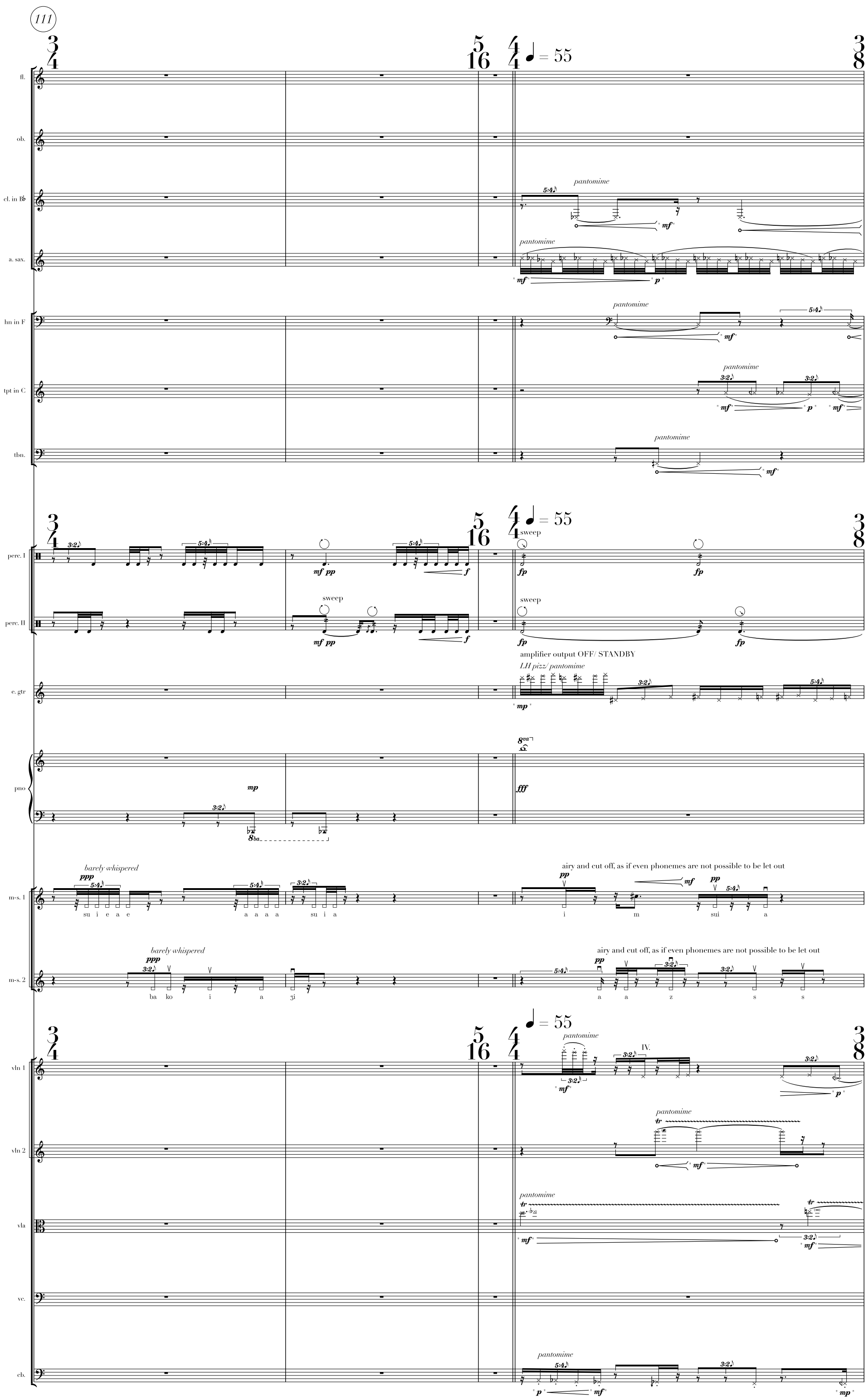
24
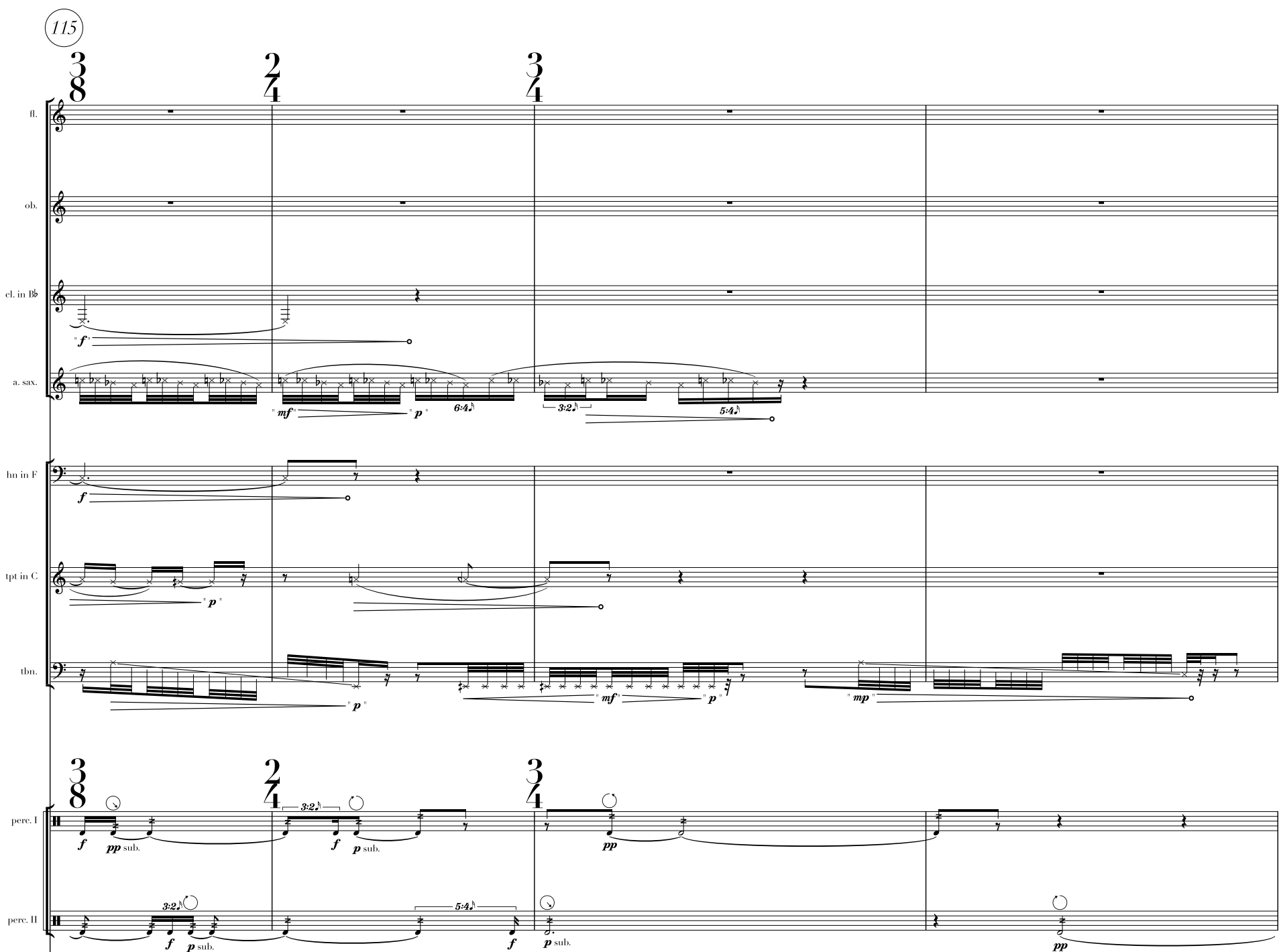

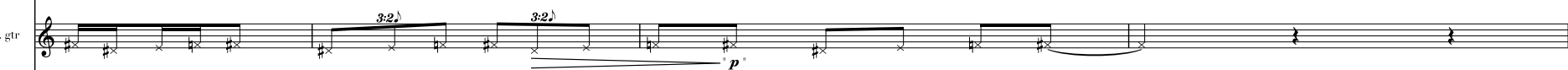

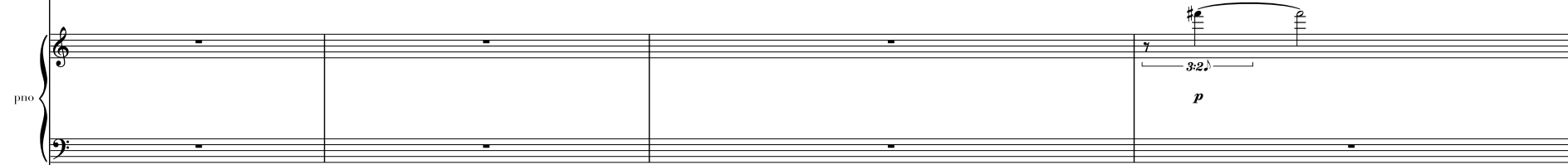

m:s.1

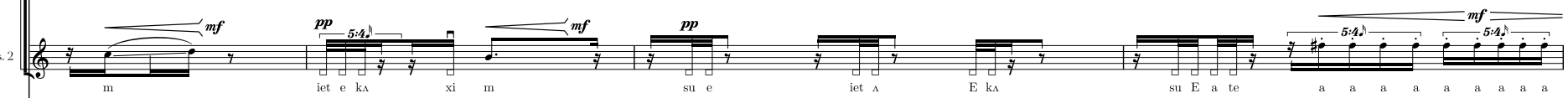

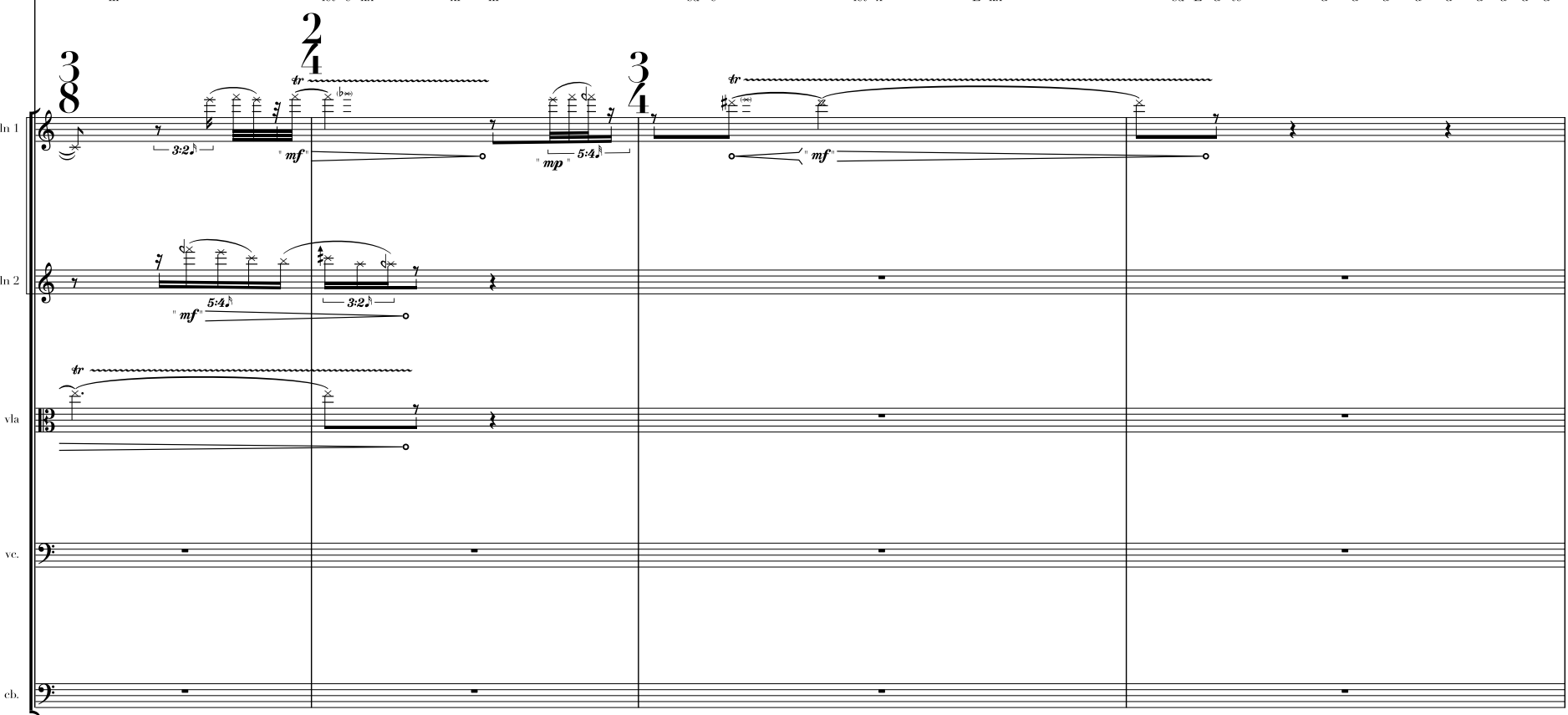



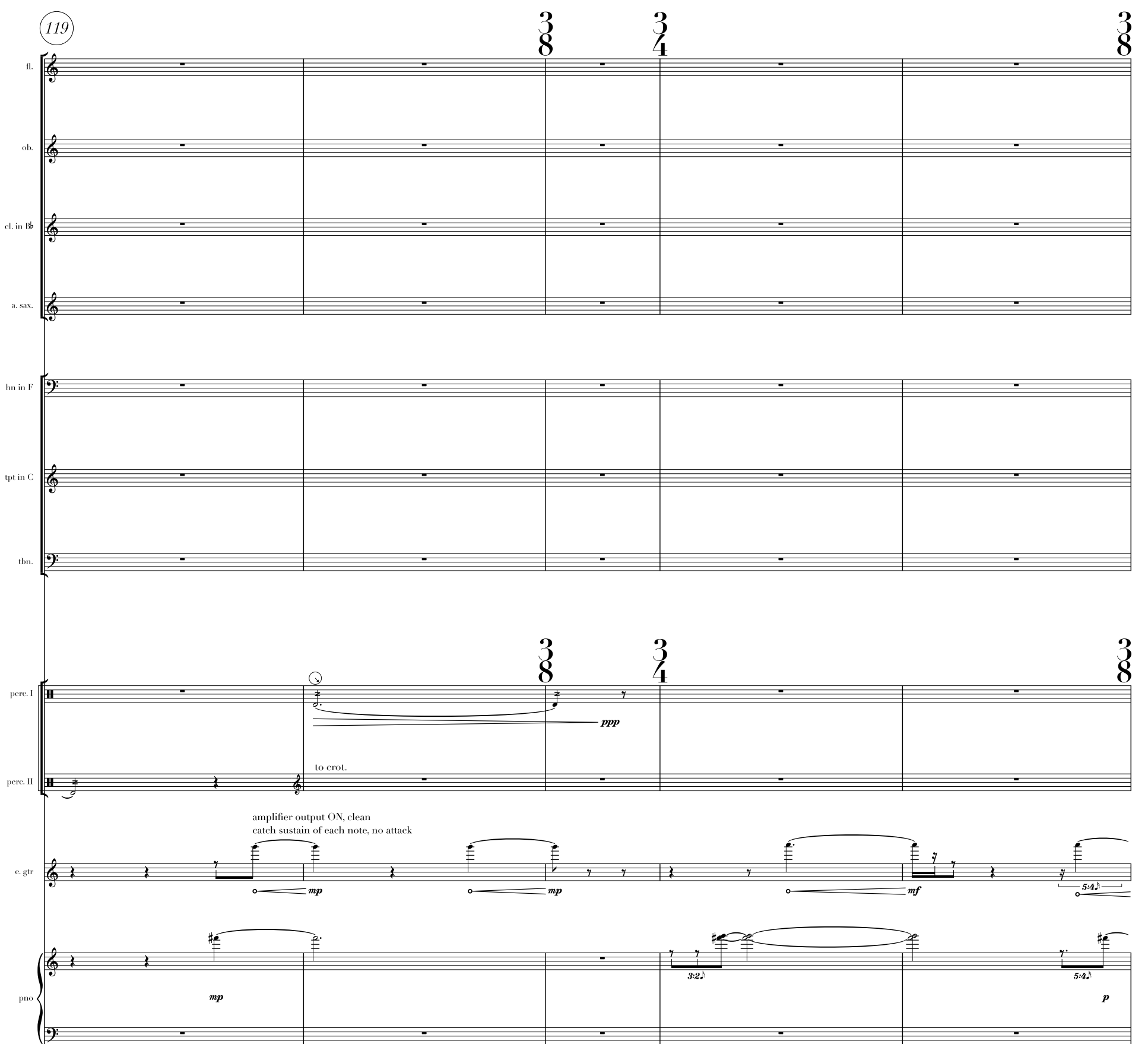

(n)

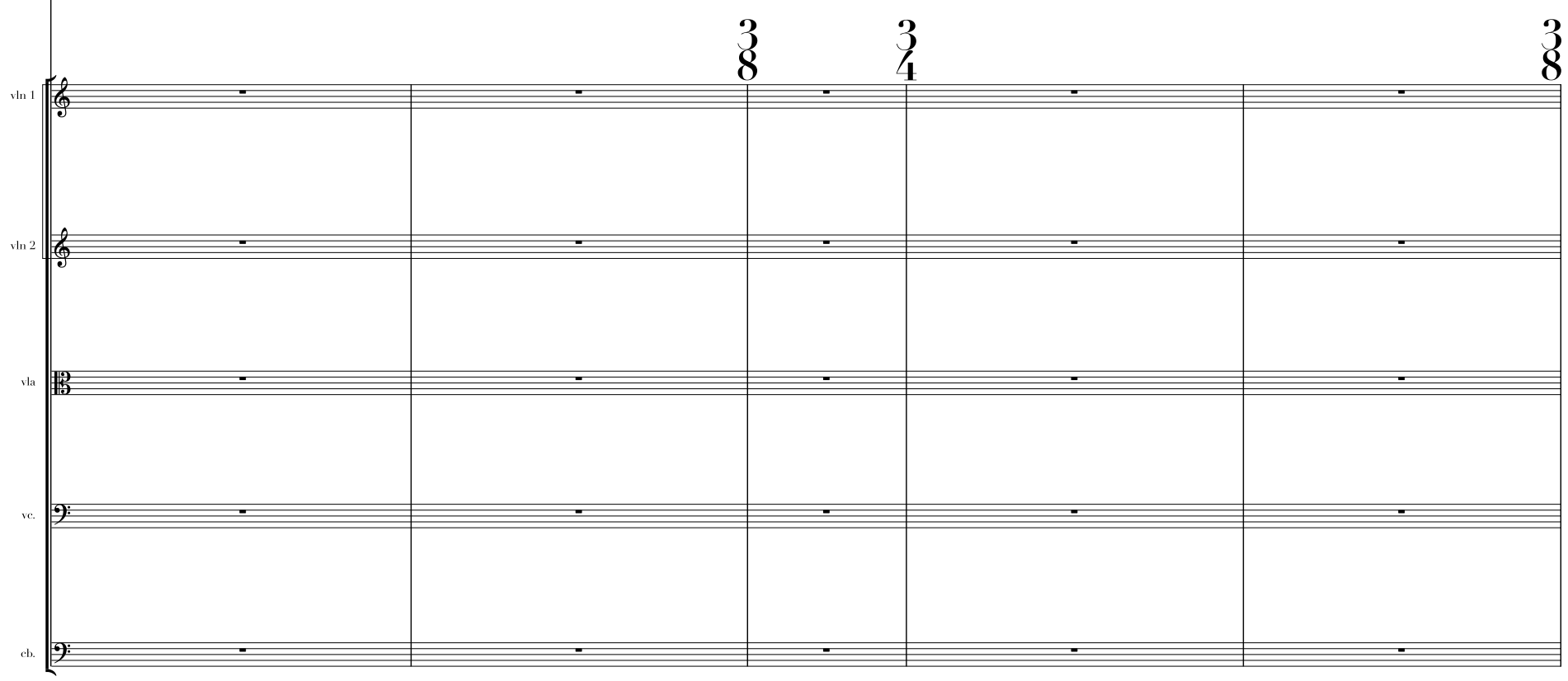


26
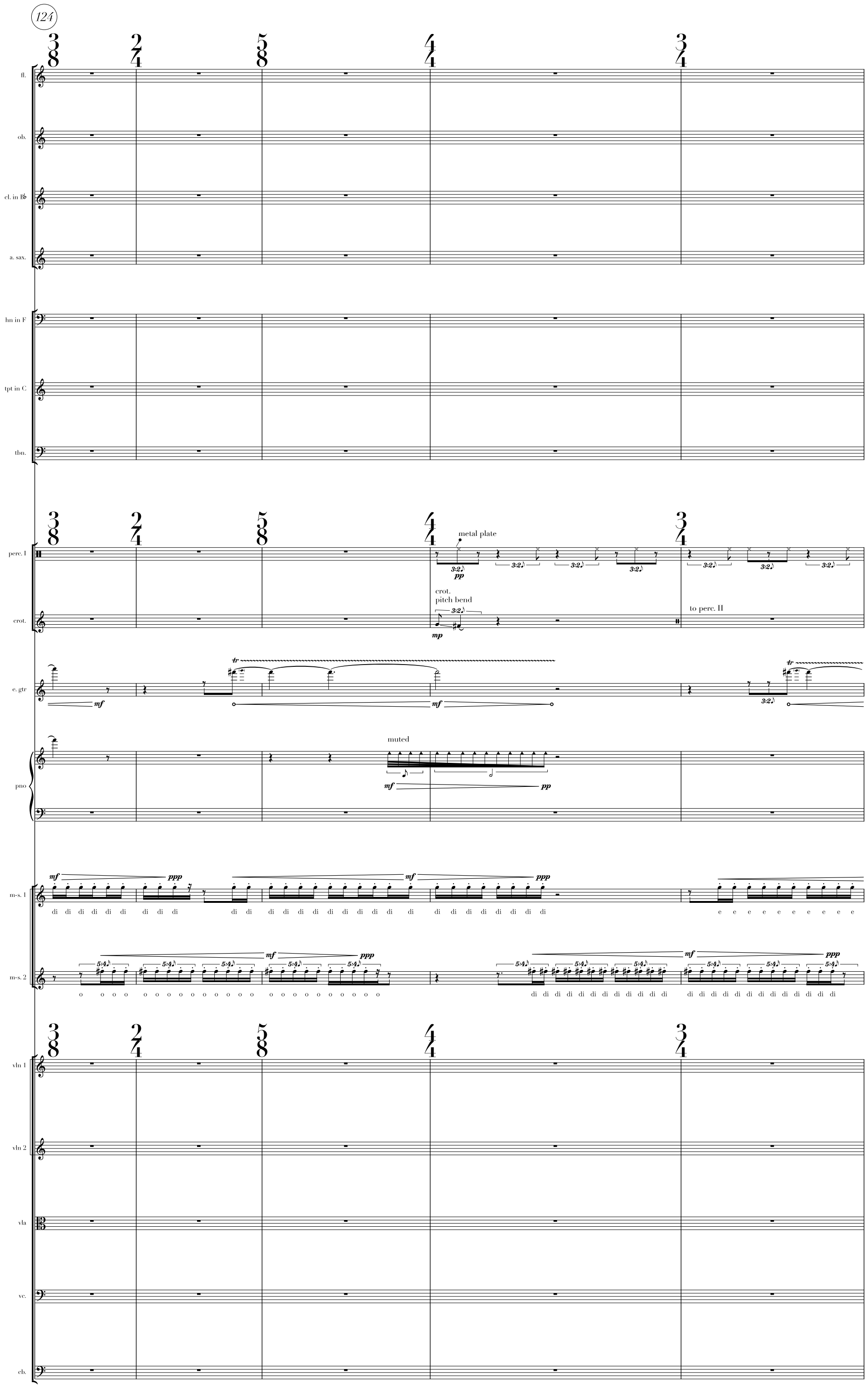

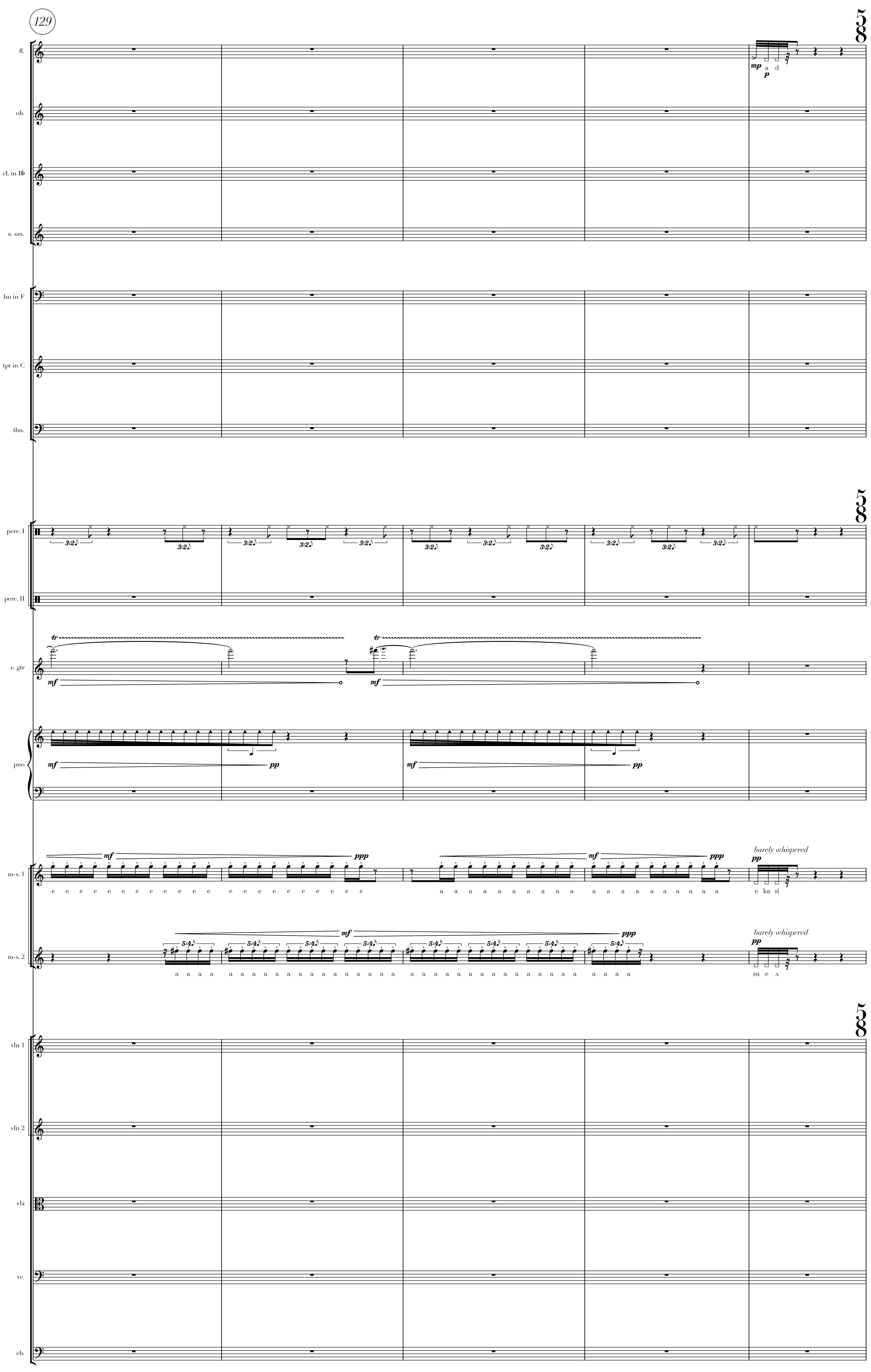
28
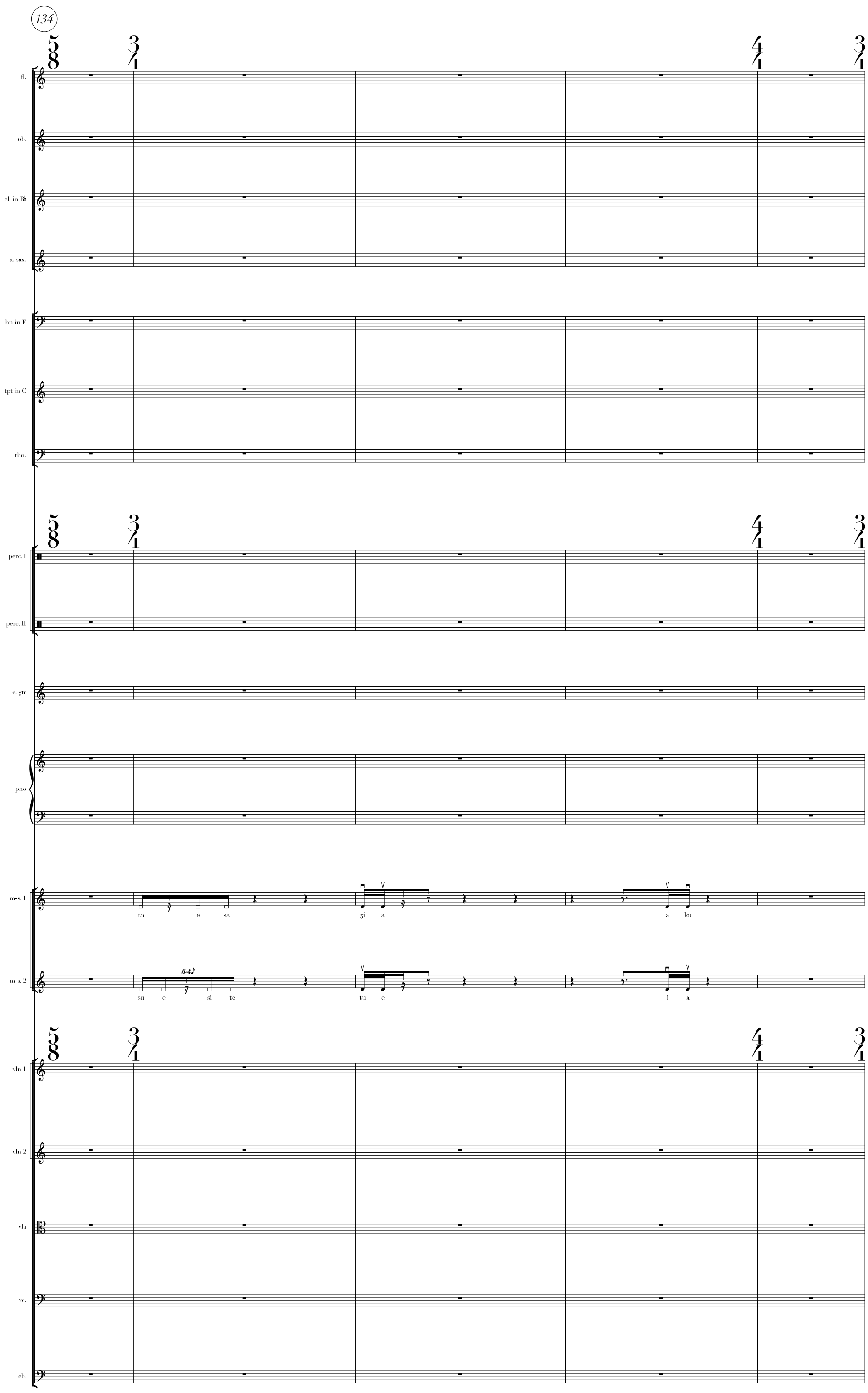

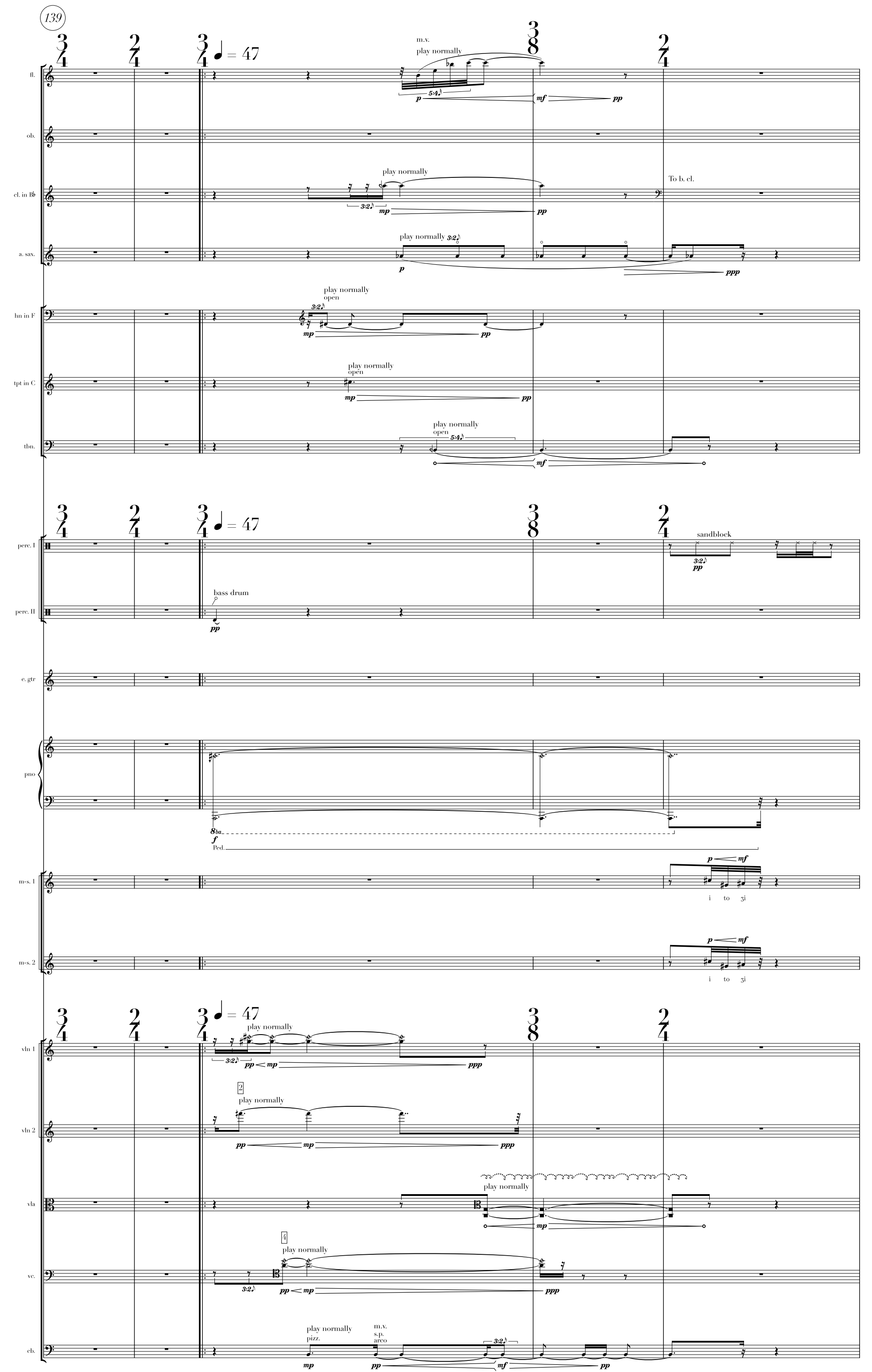

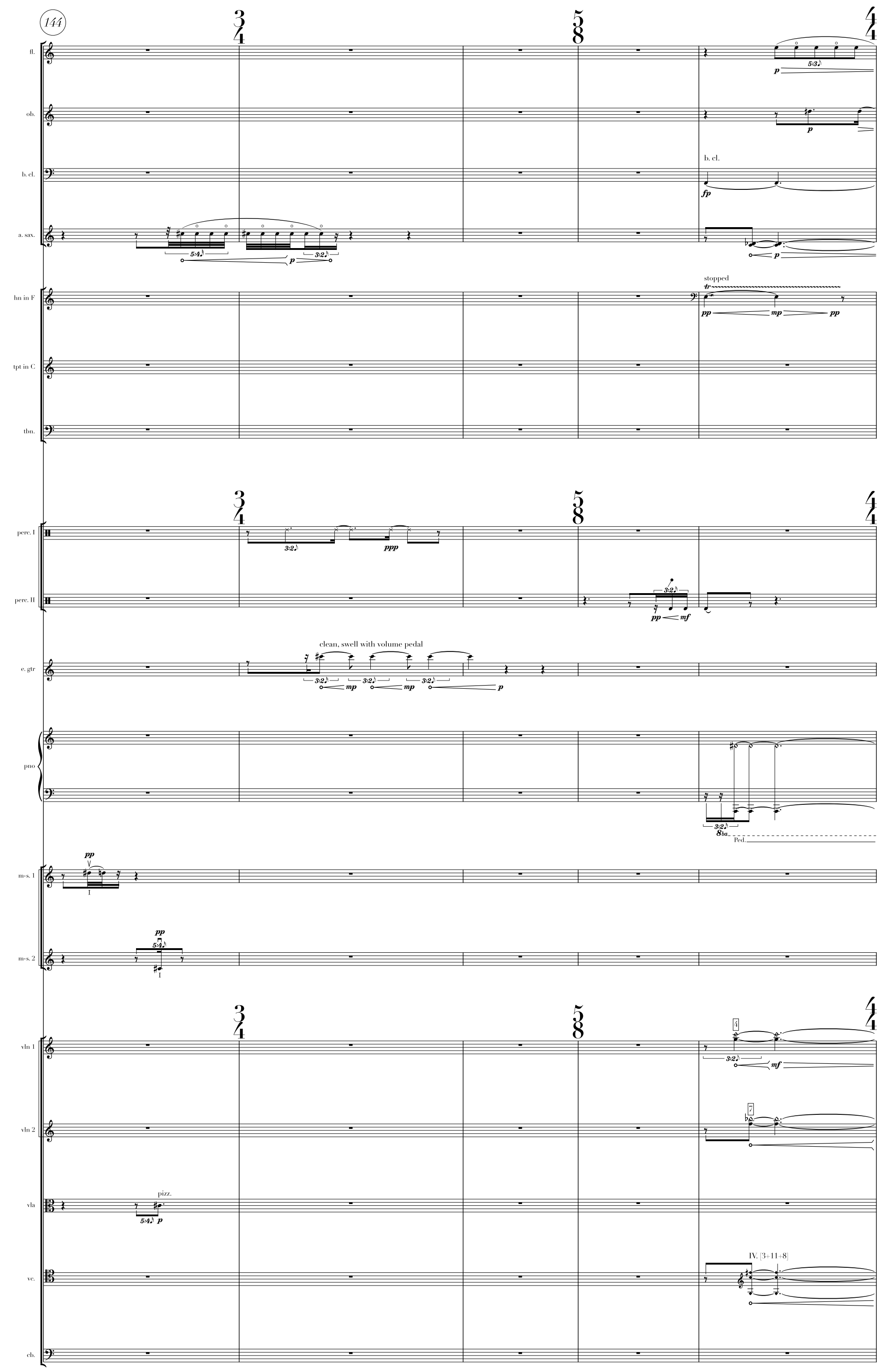

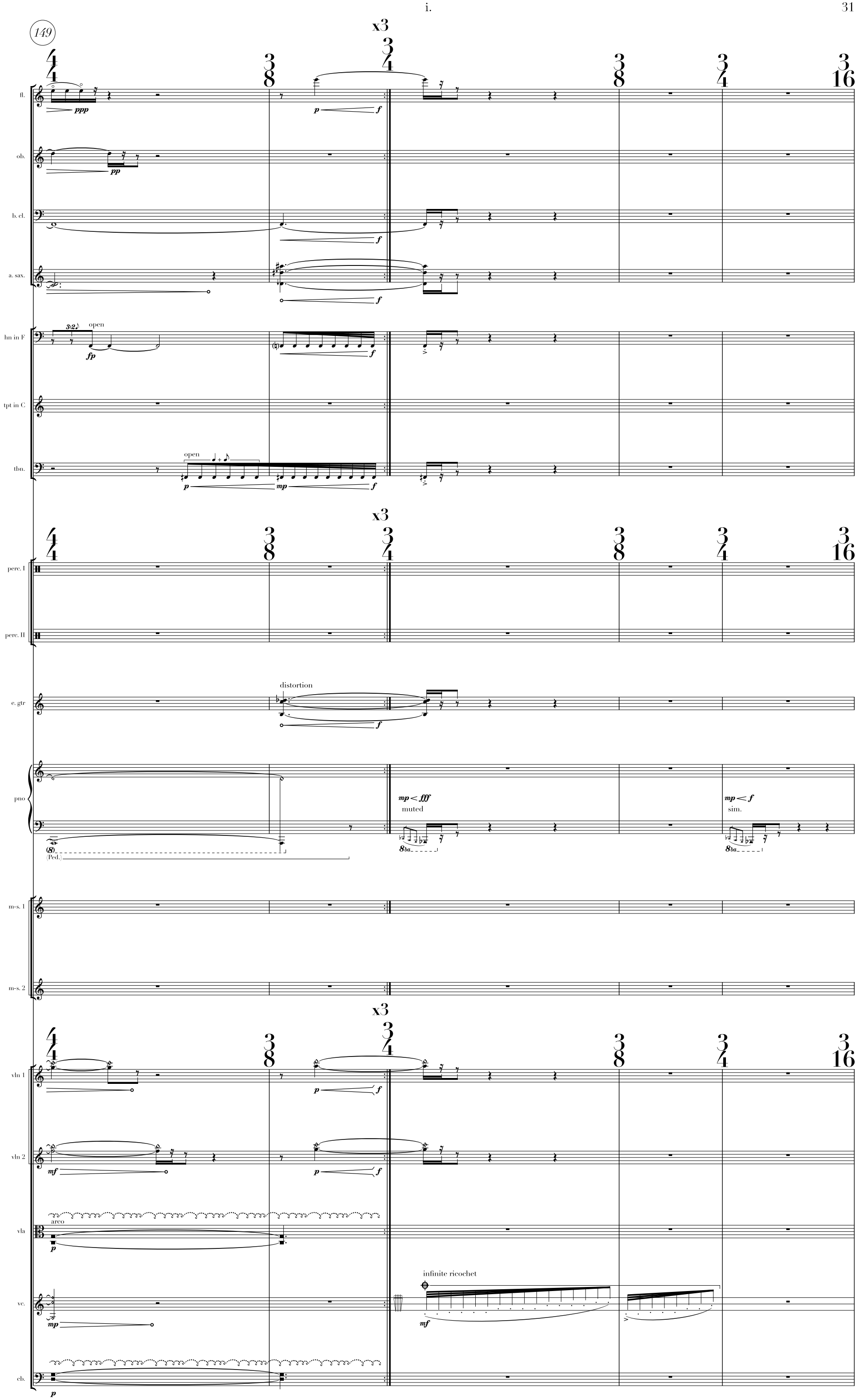
32
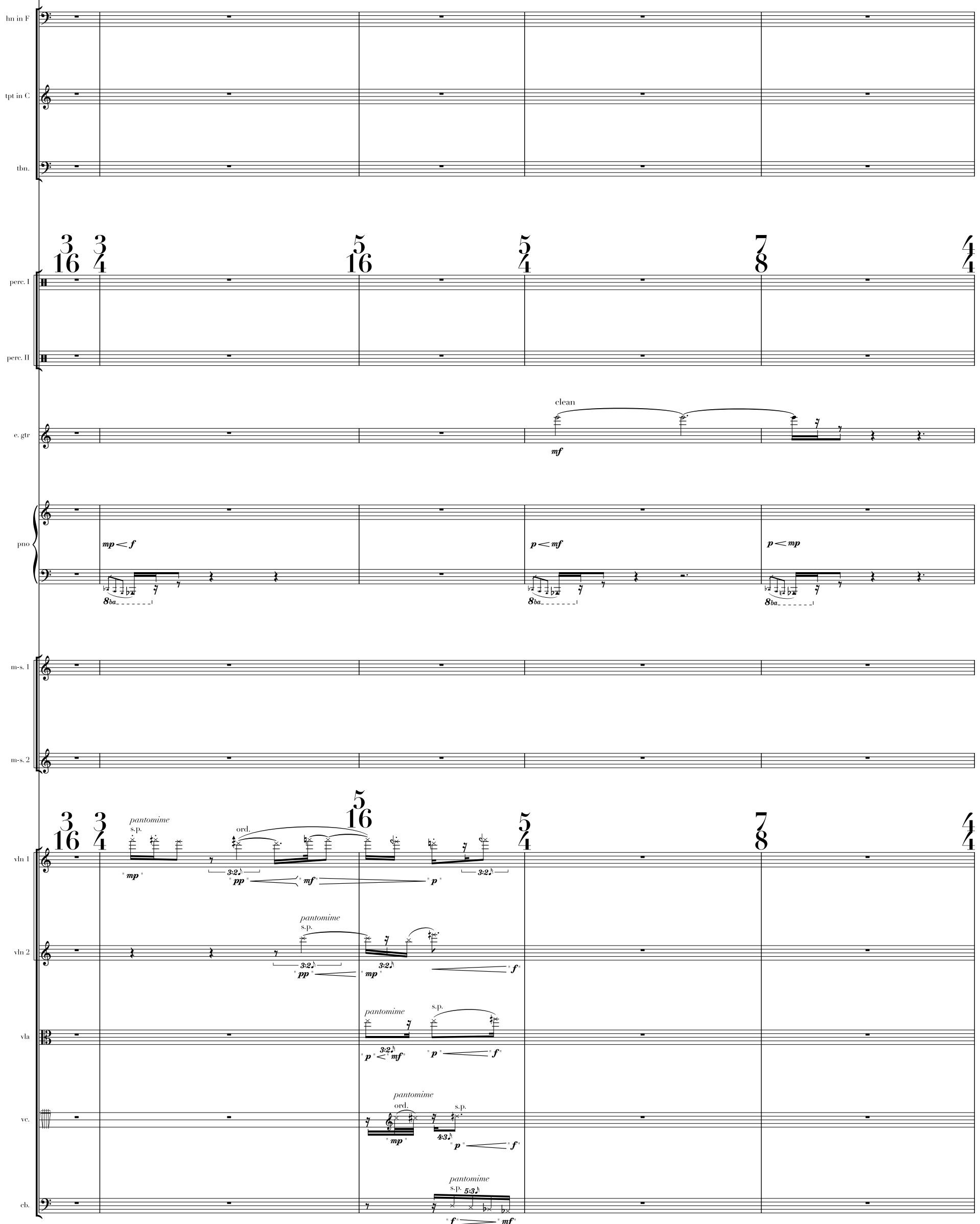

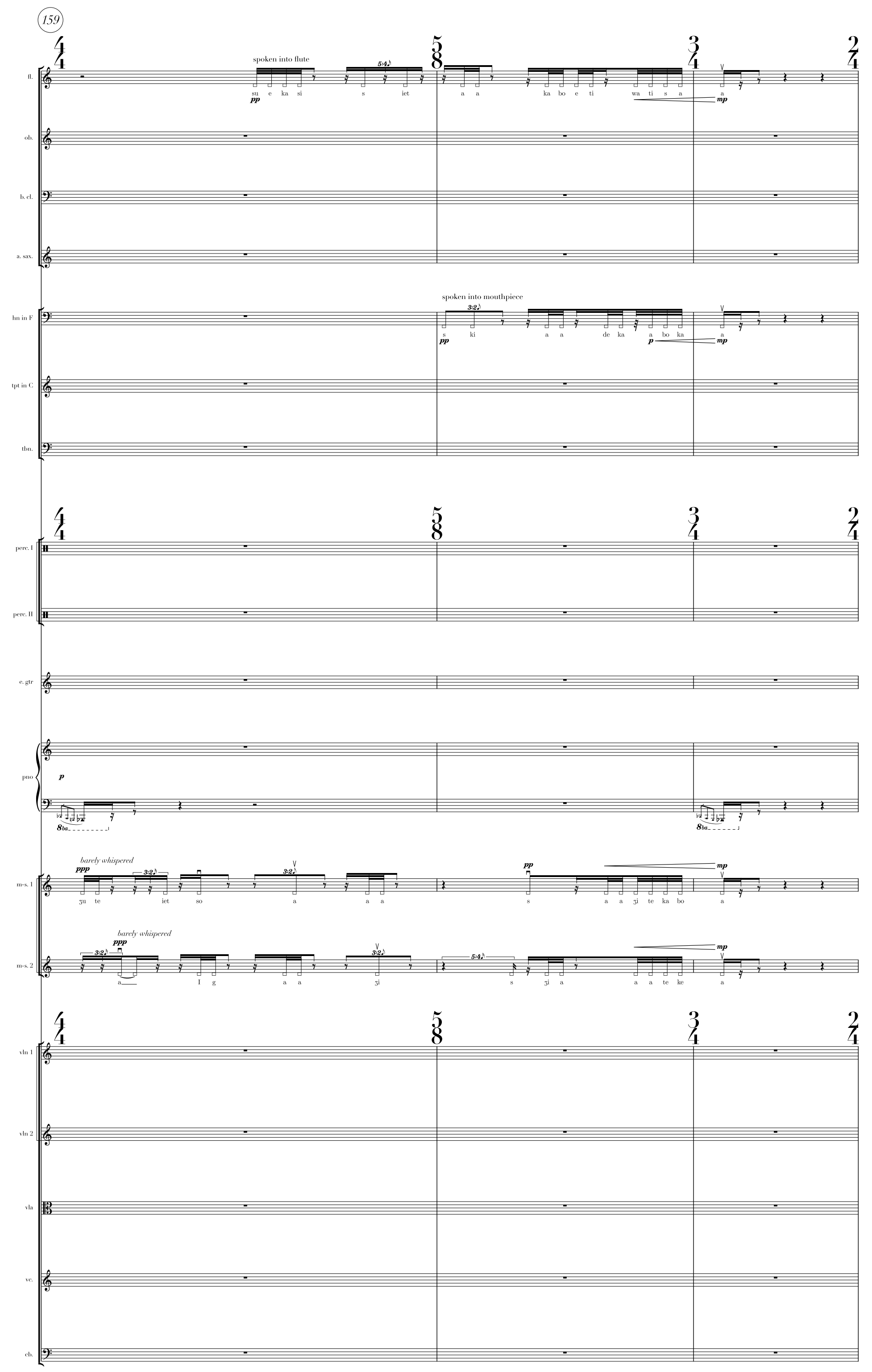

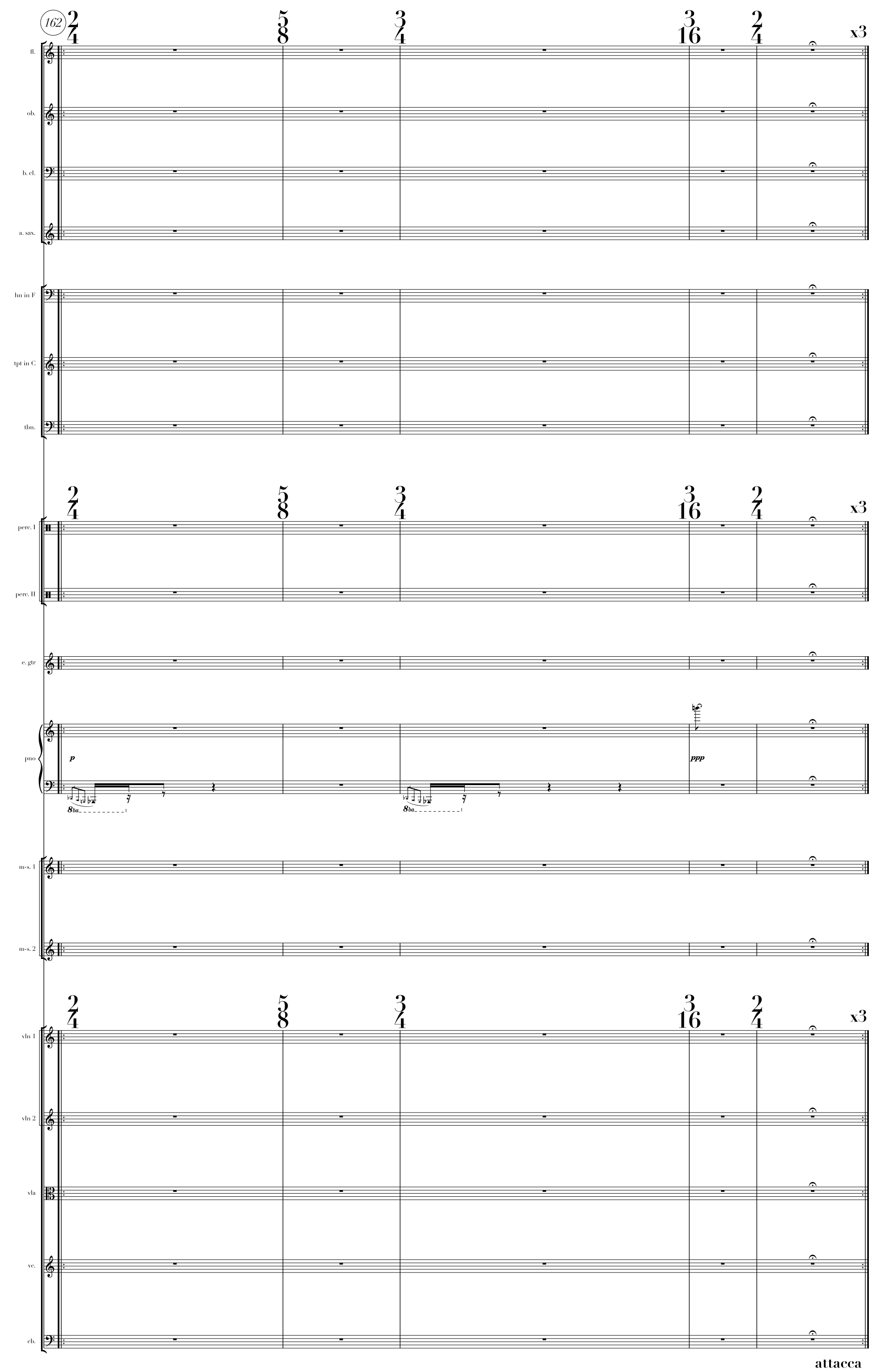

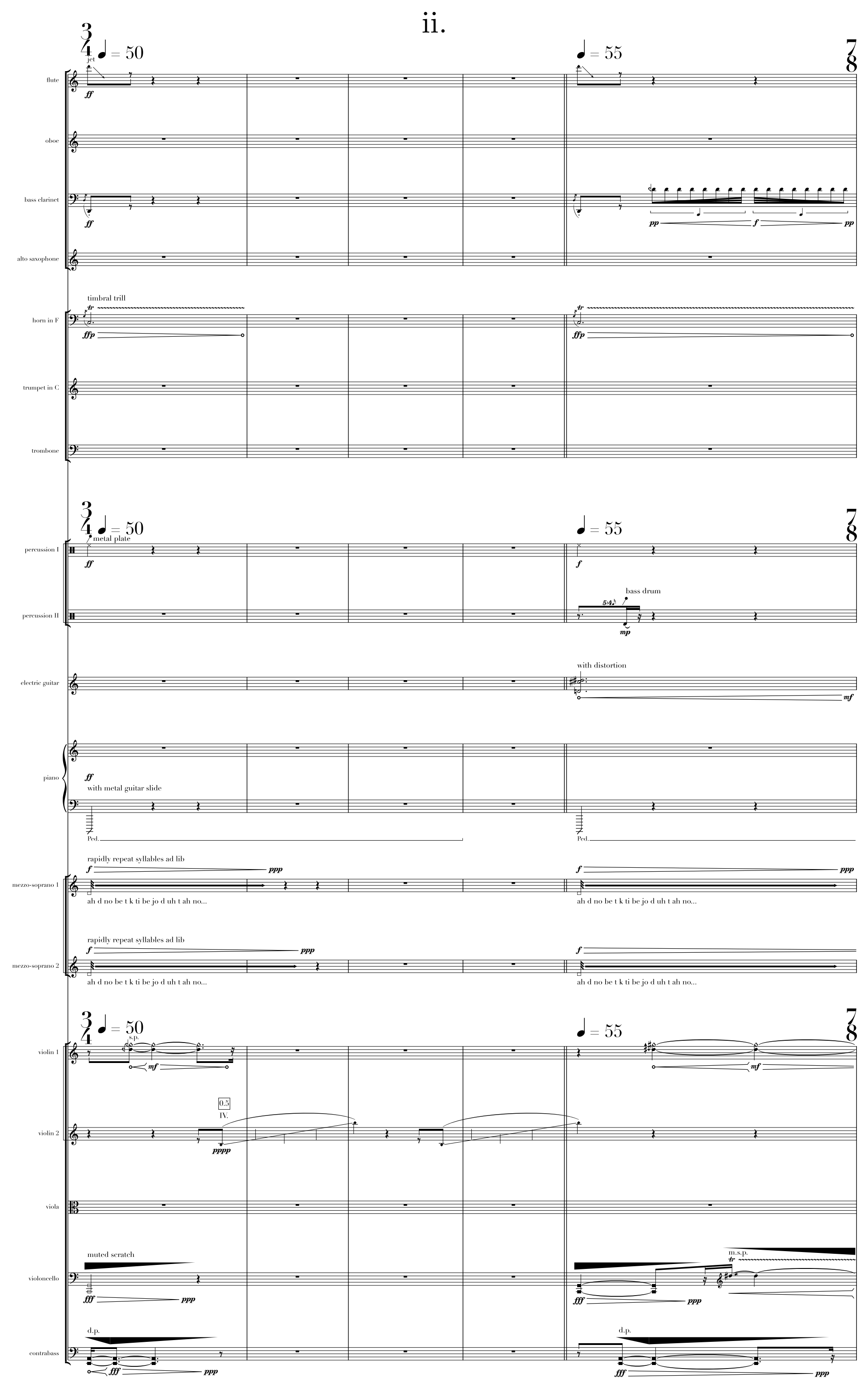
36

ii.
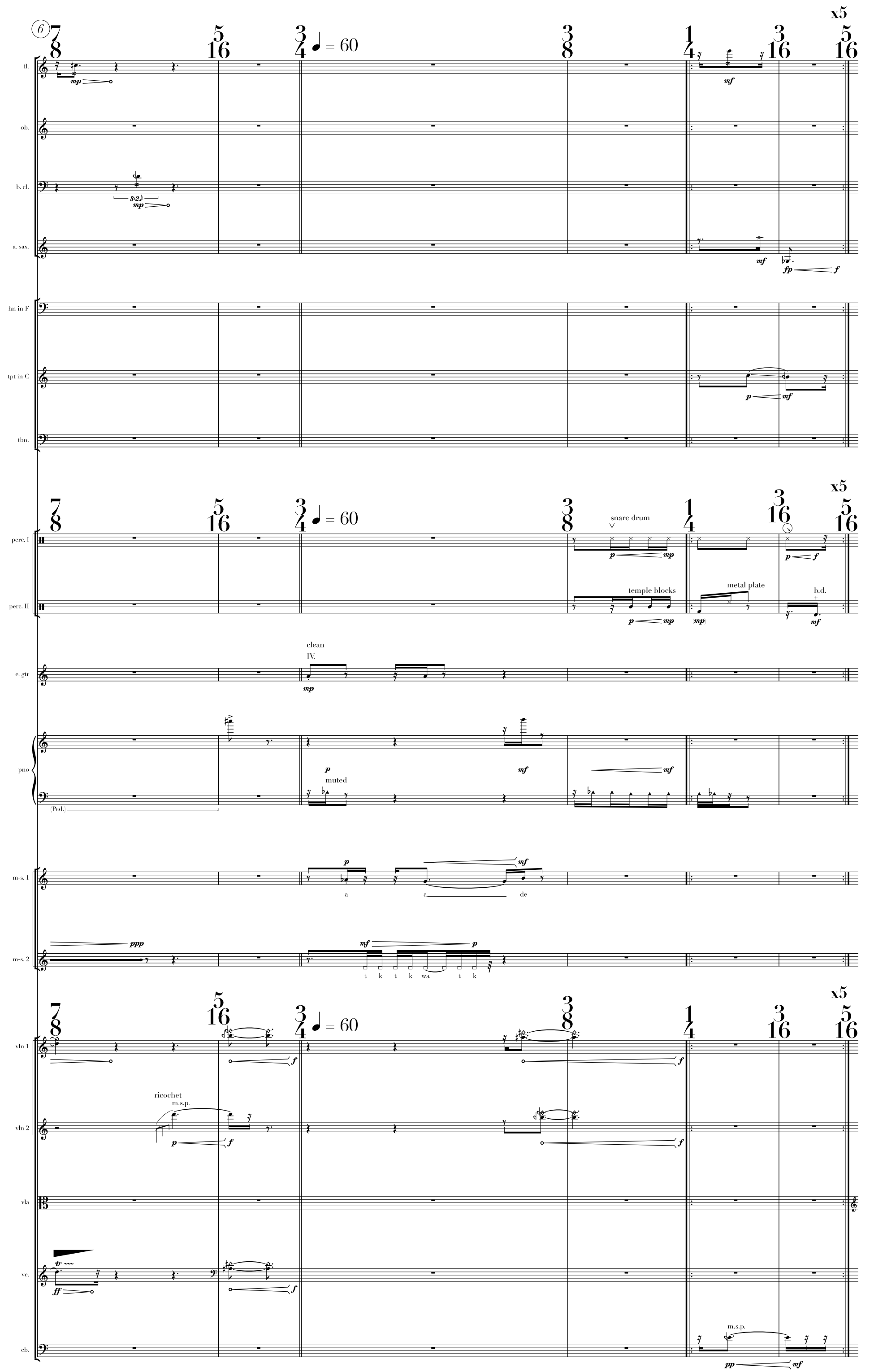

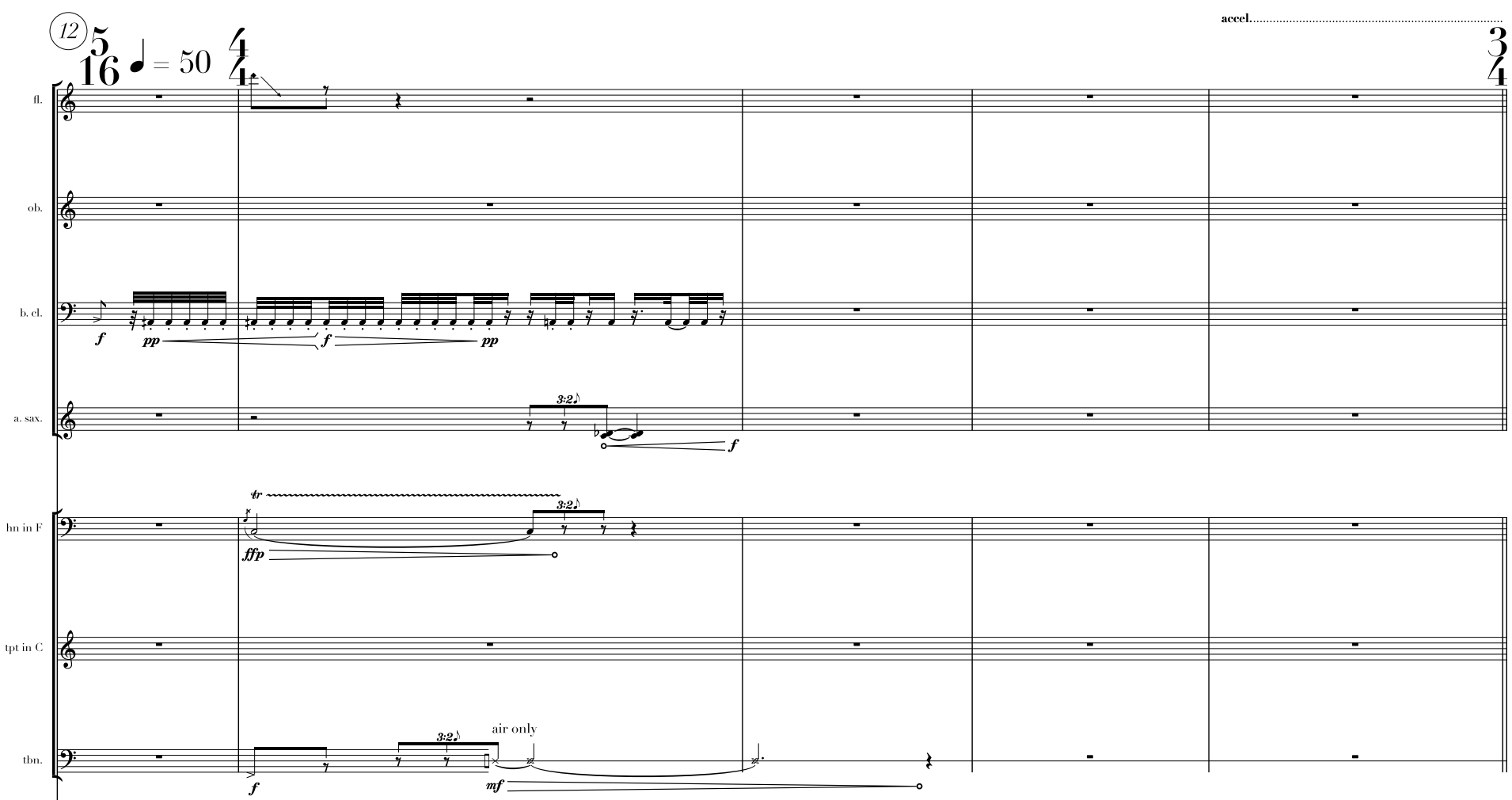

$56^{5} \cdot=504$

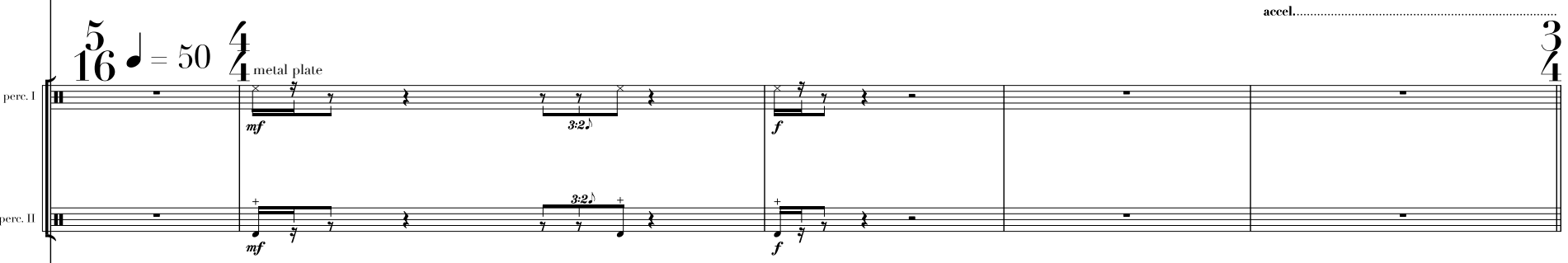

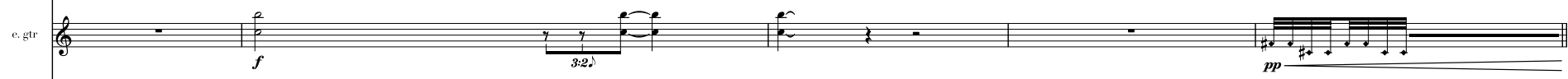
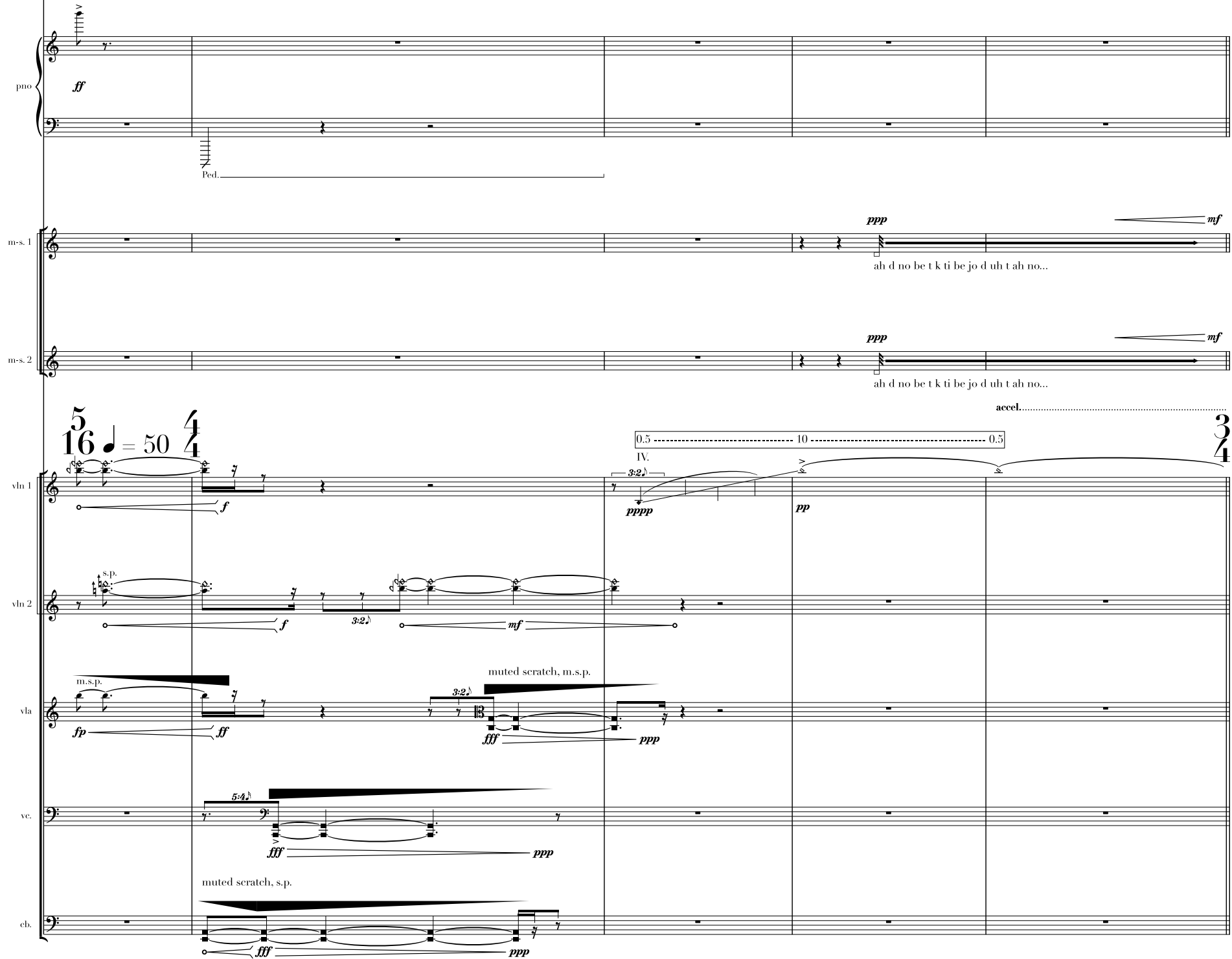

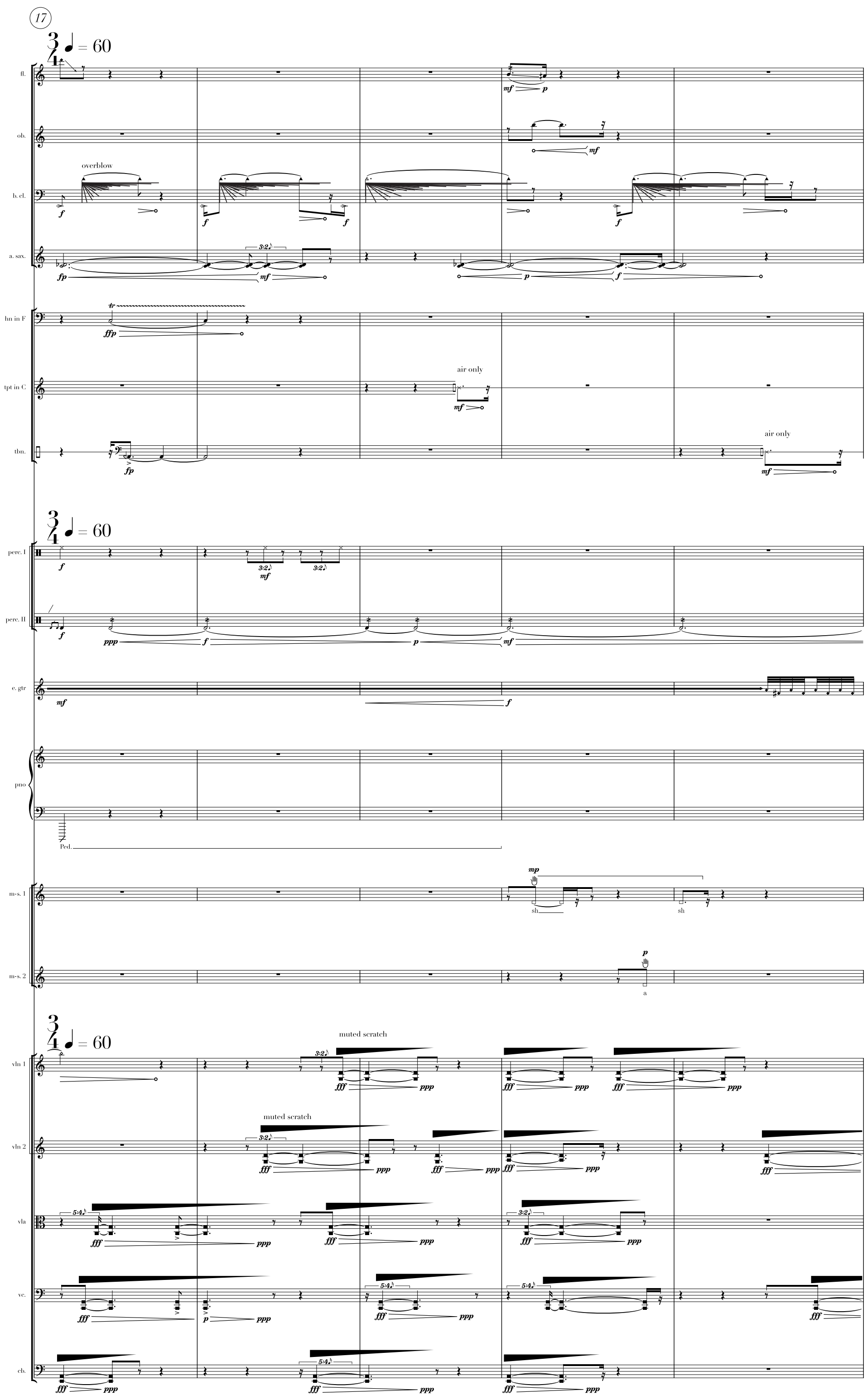

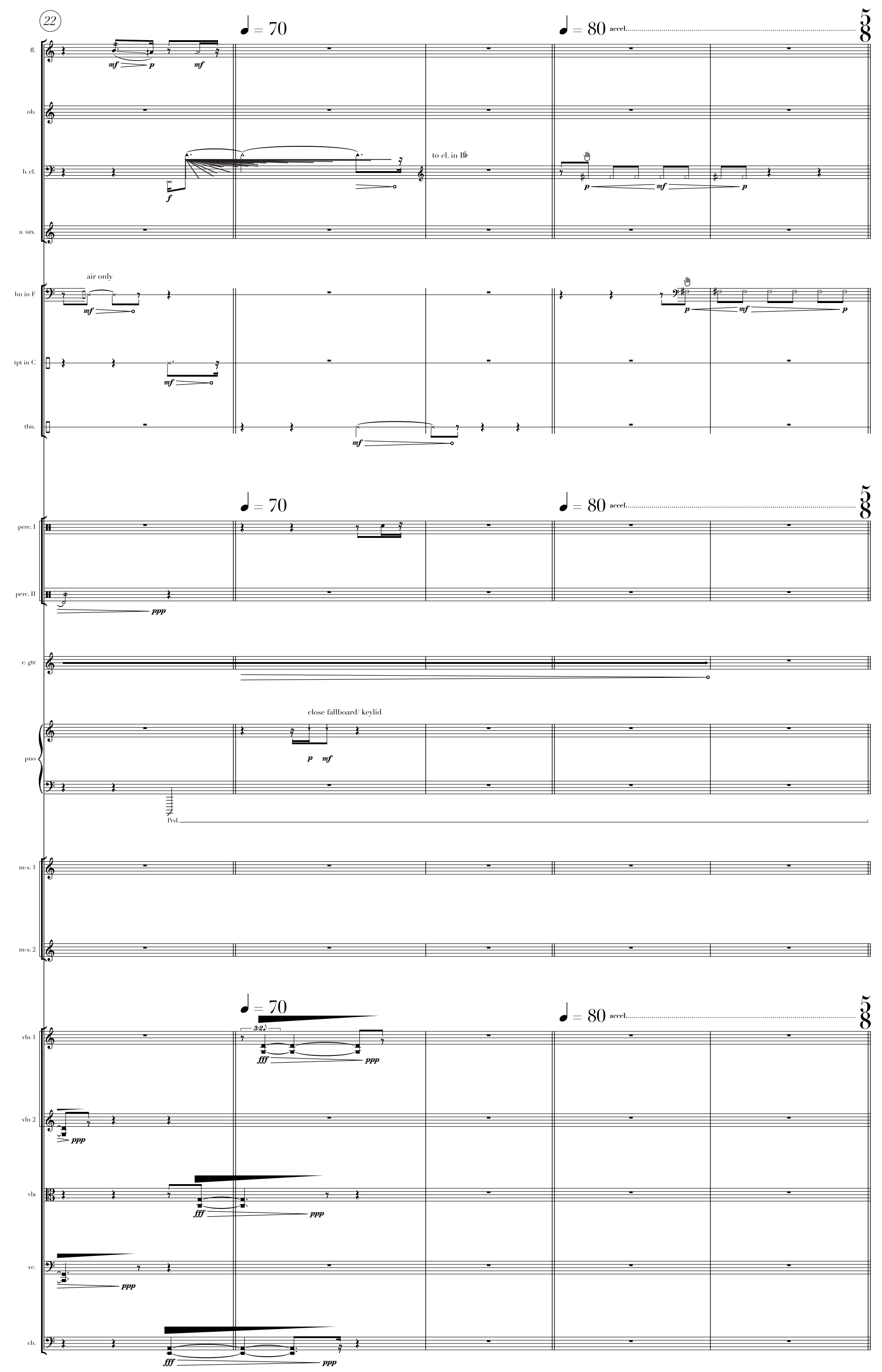
40
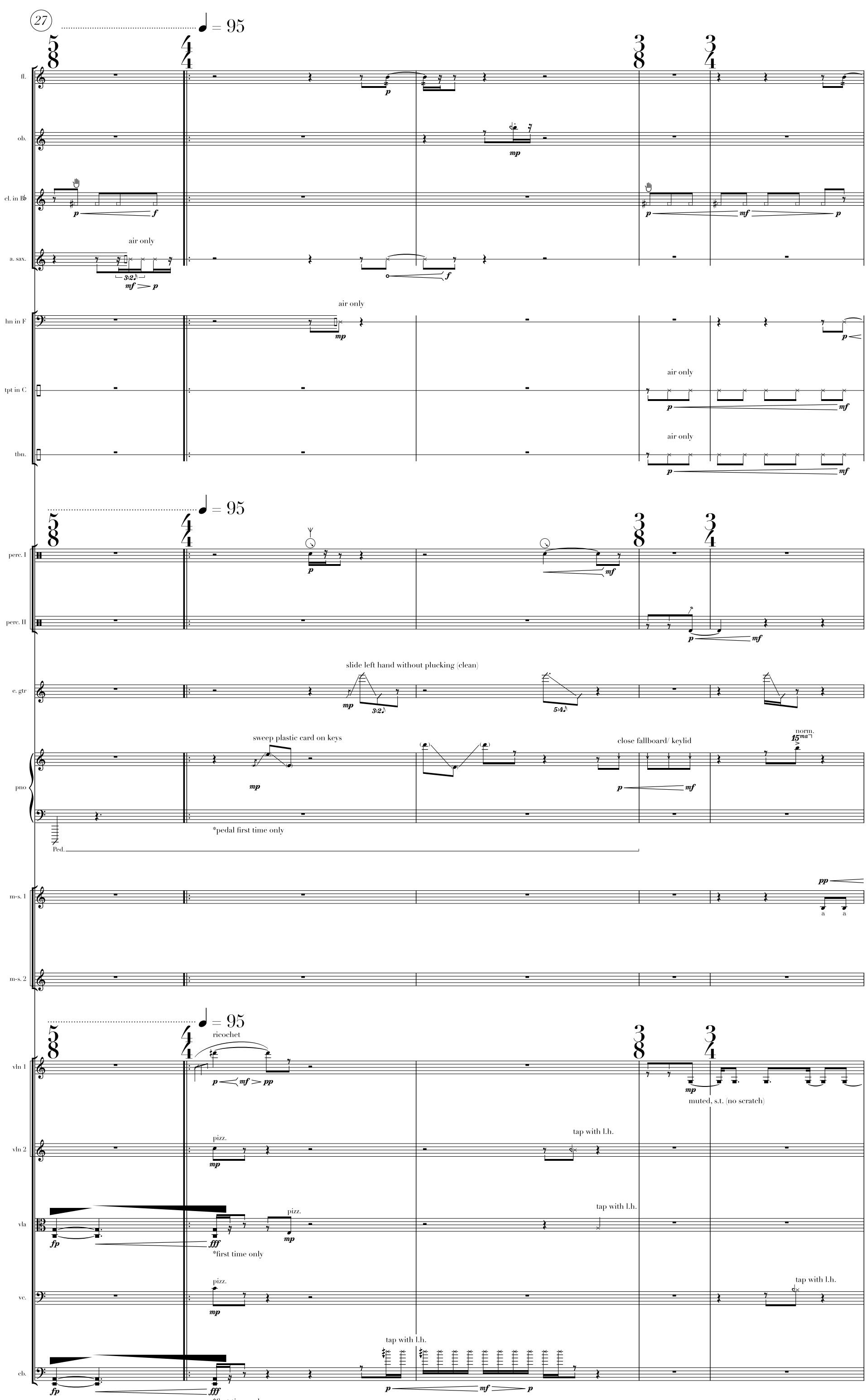
ii.

41
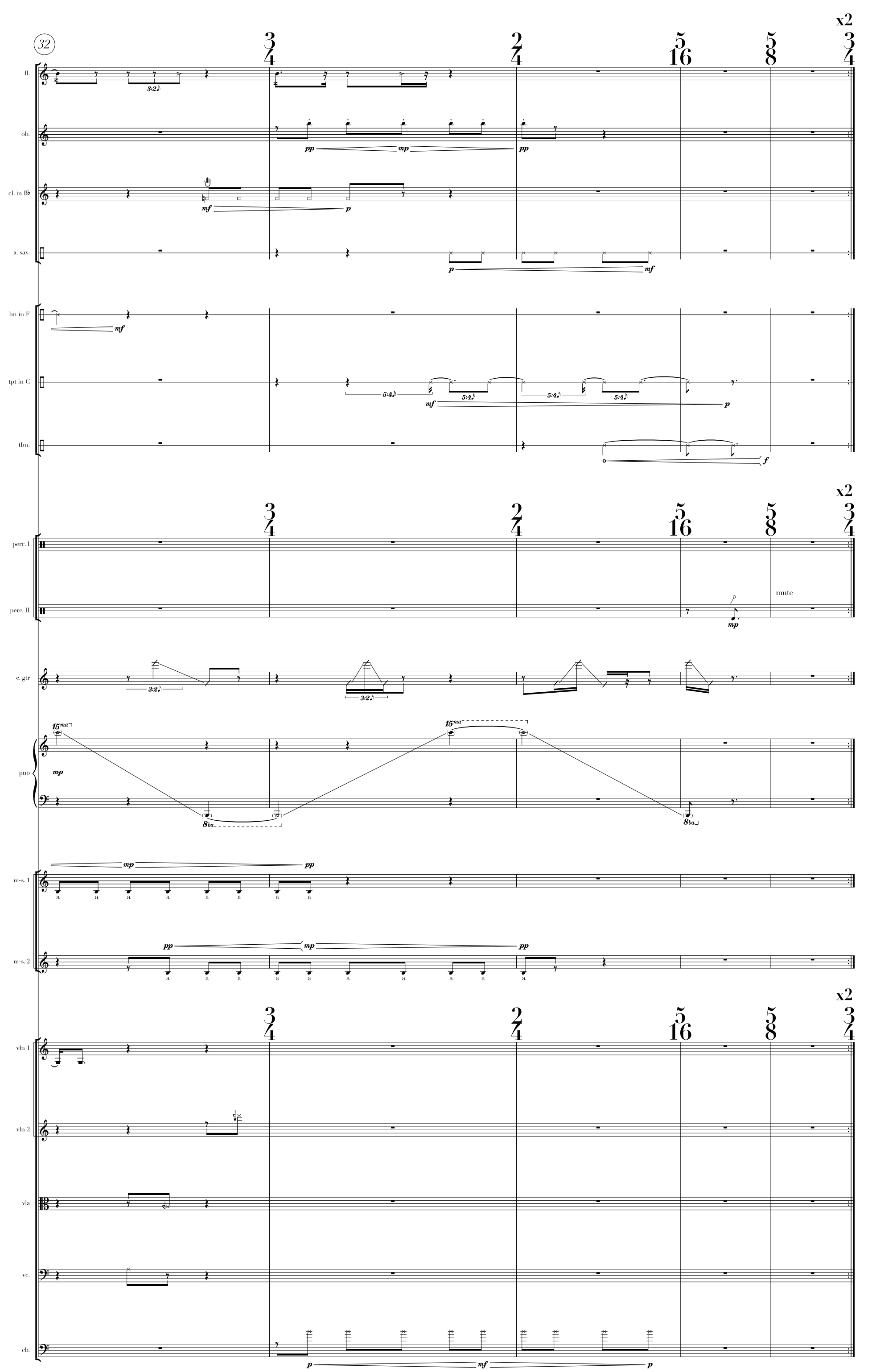
42

ii.
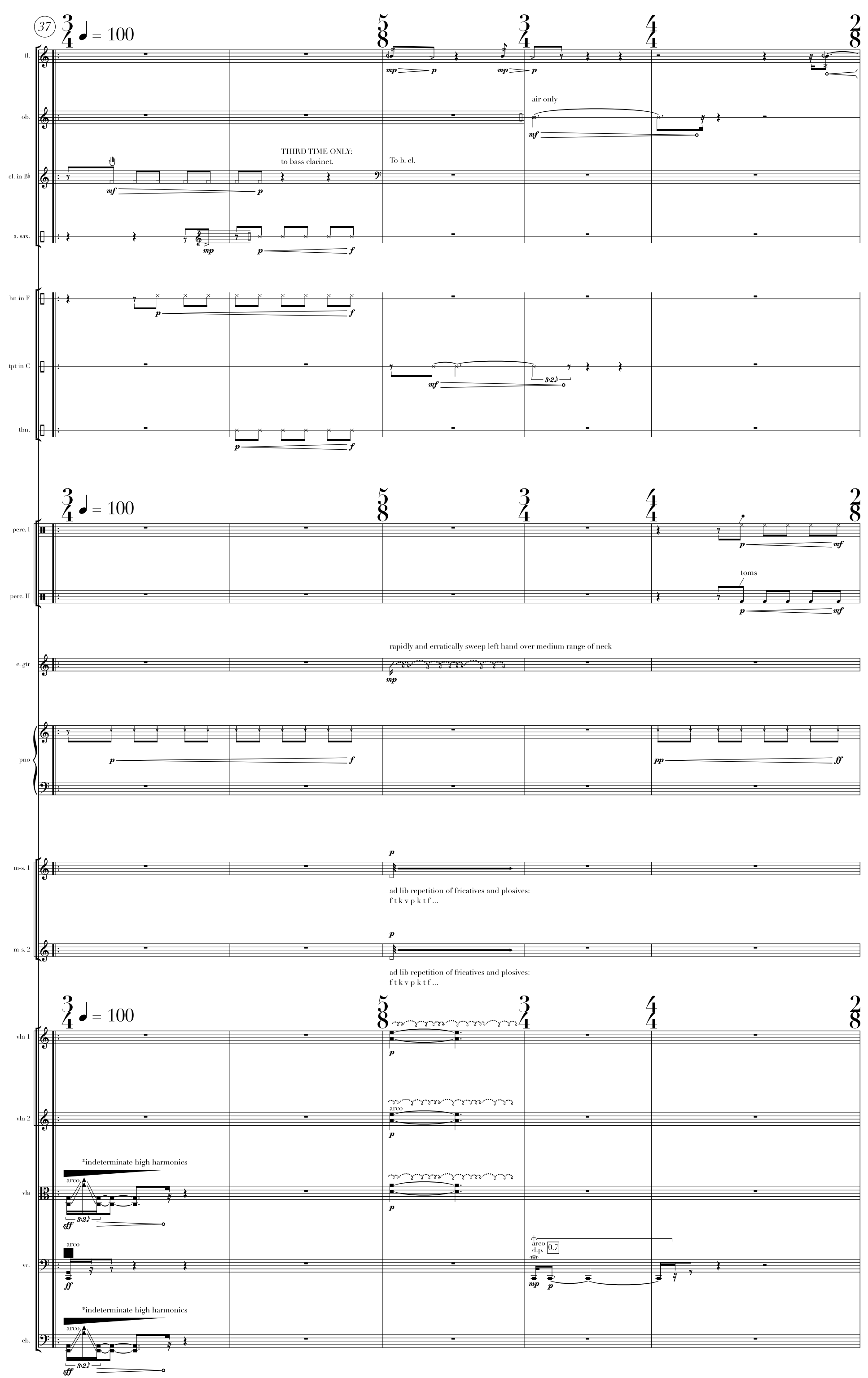

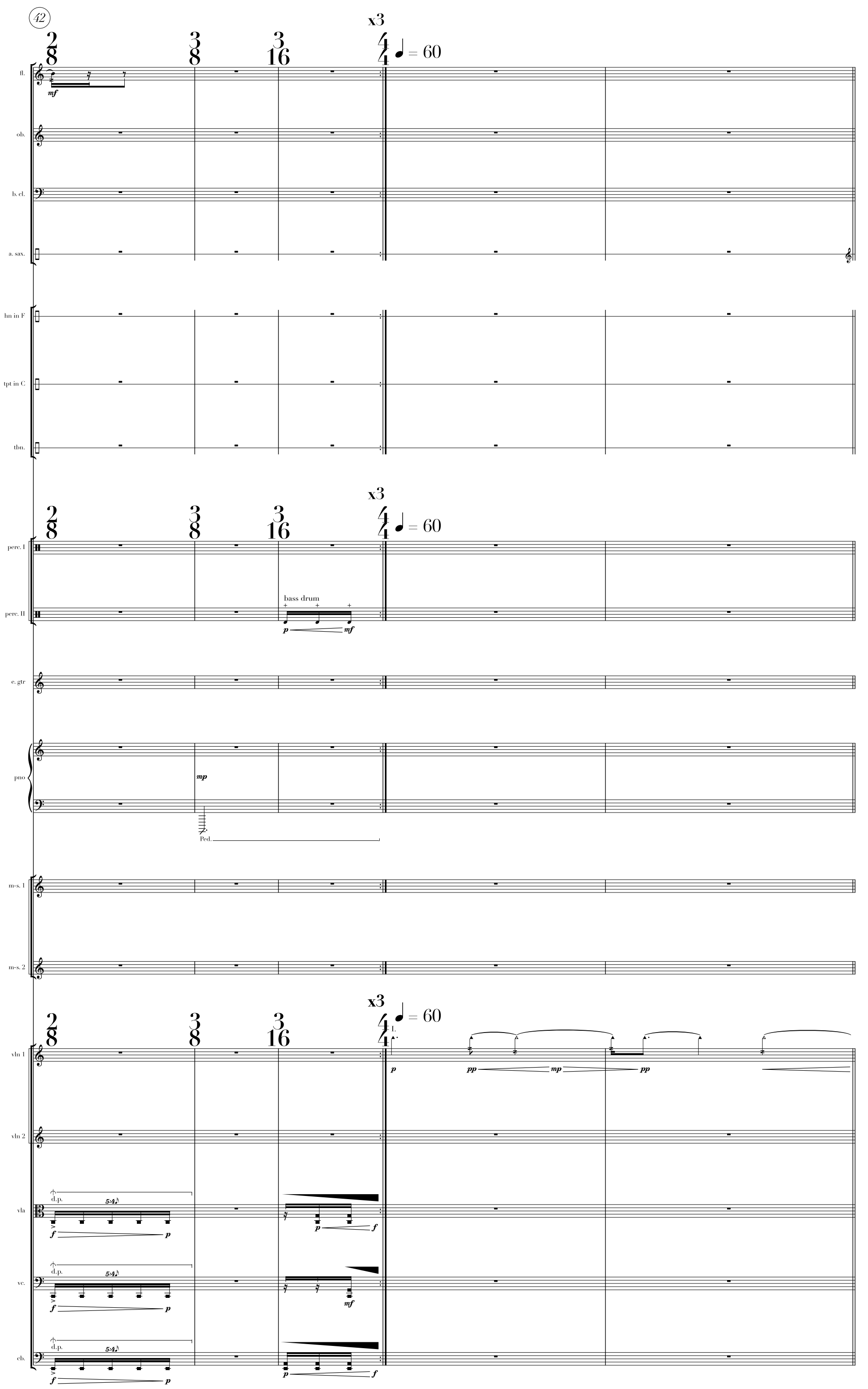

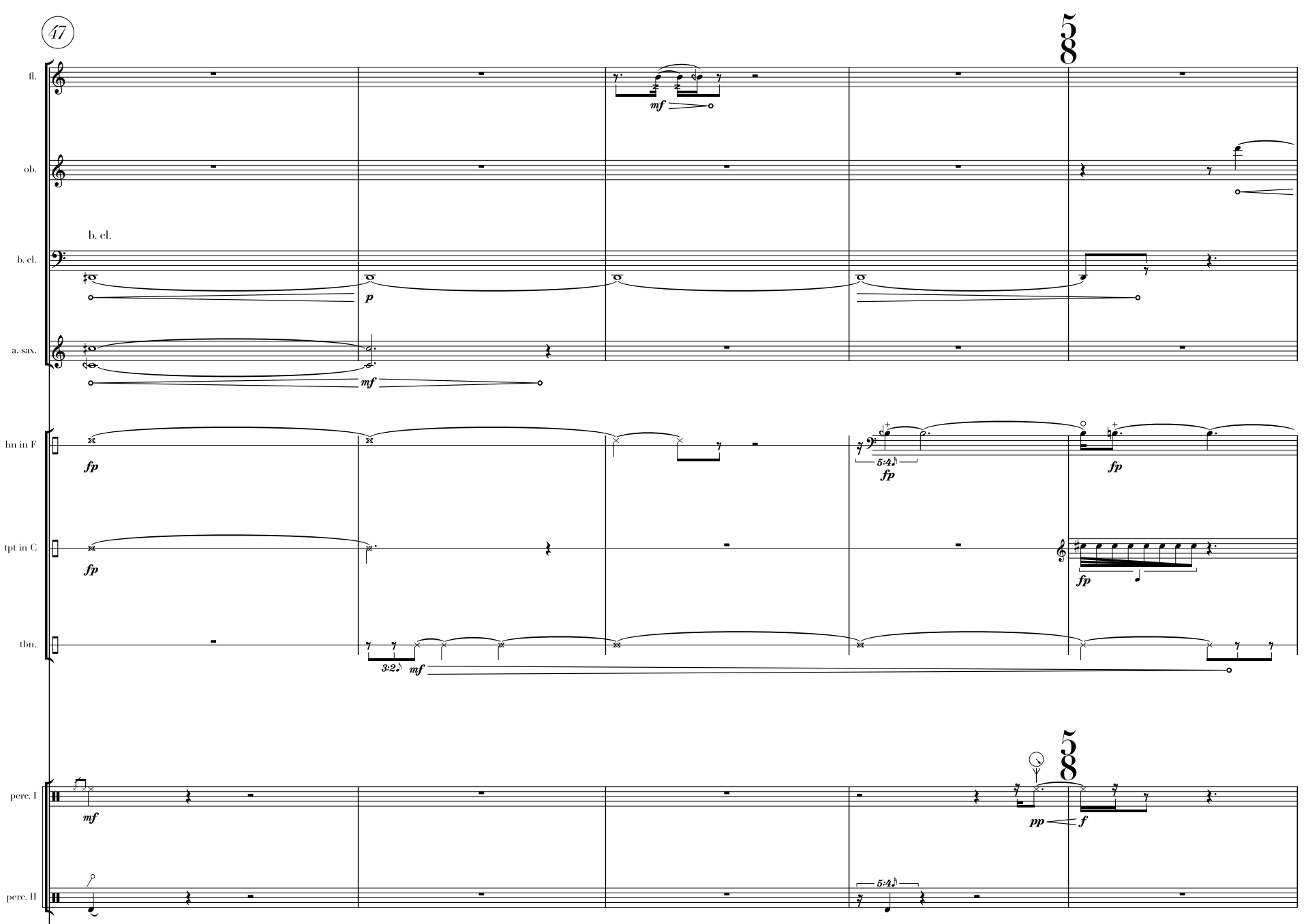

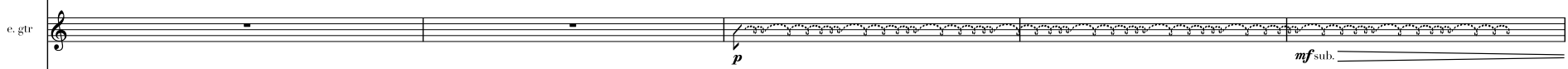

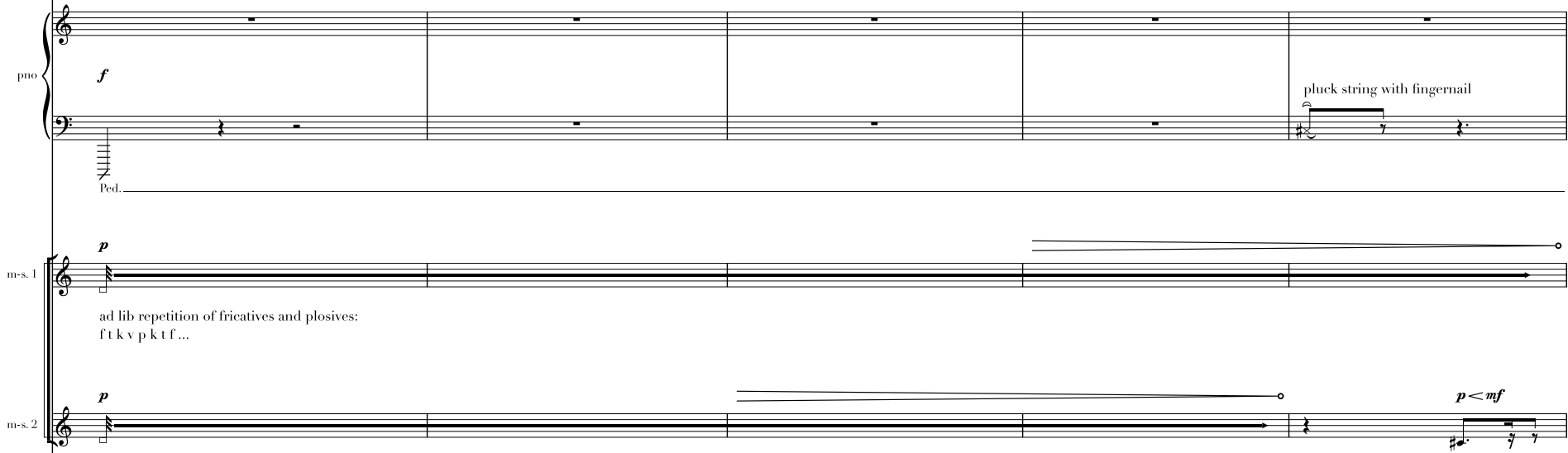

ad lib repetition of fricatives and plosives:
$\mathrm{ft} k \mathrm{k} v \mathrm{k}$ t $\mathrm{f}$...

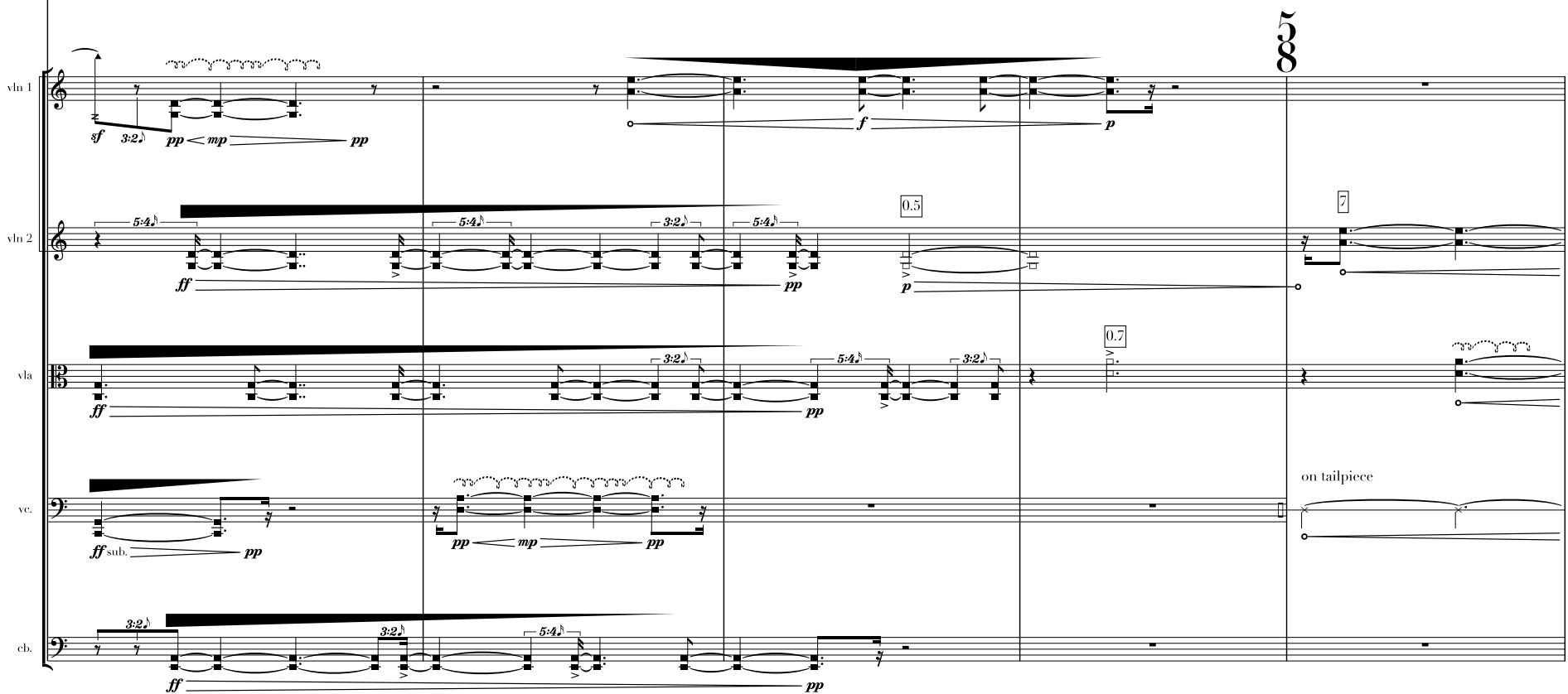



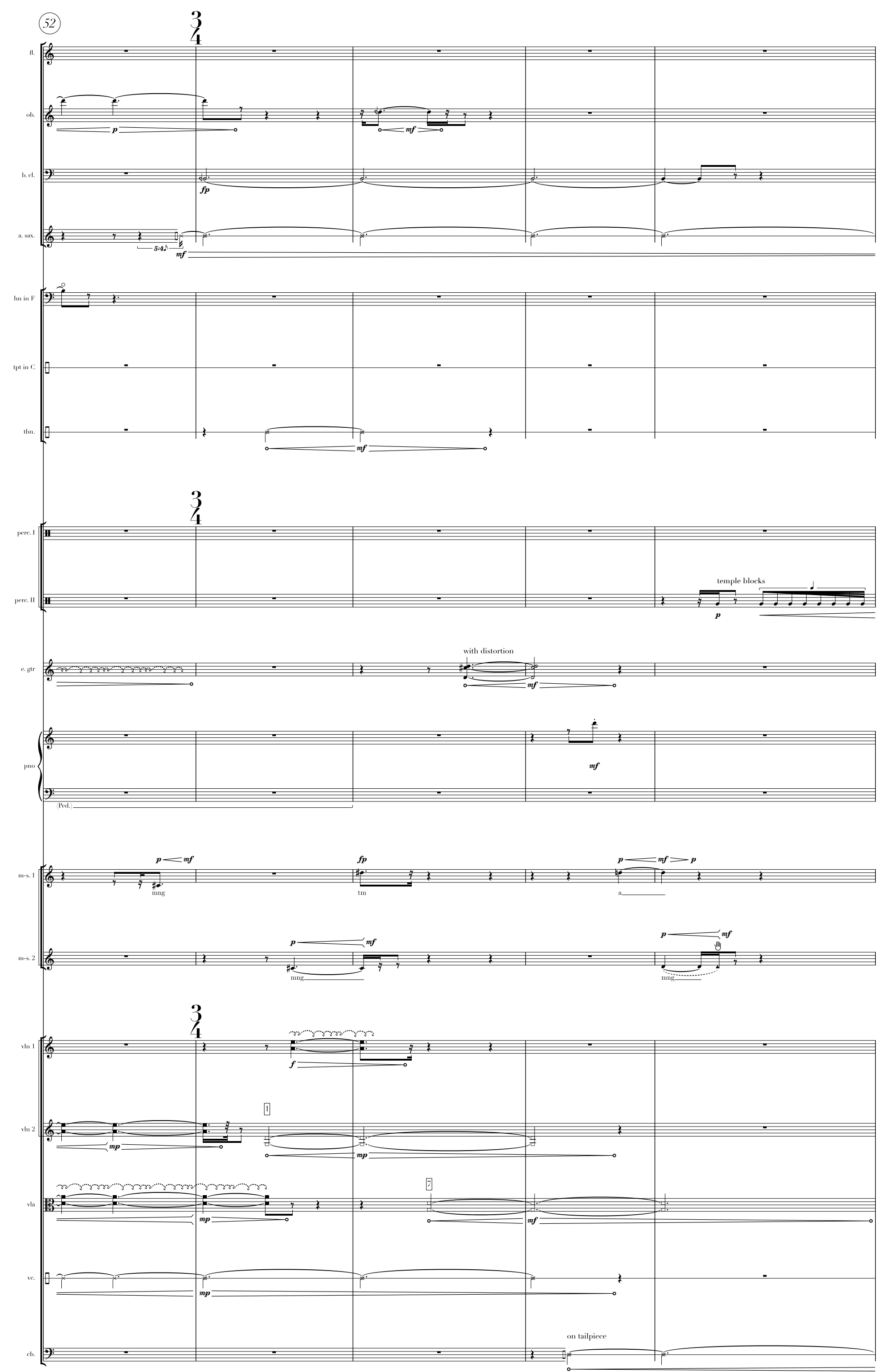
(57)
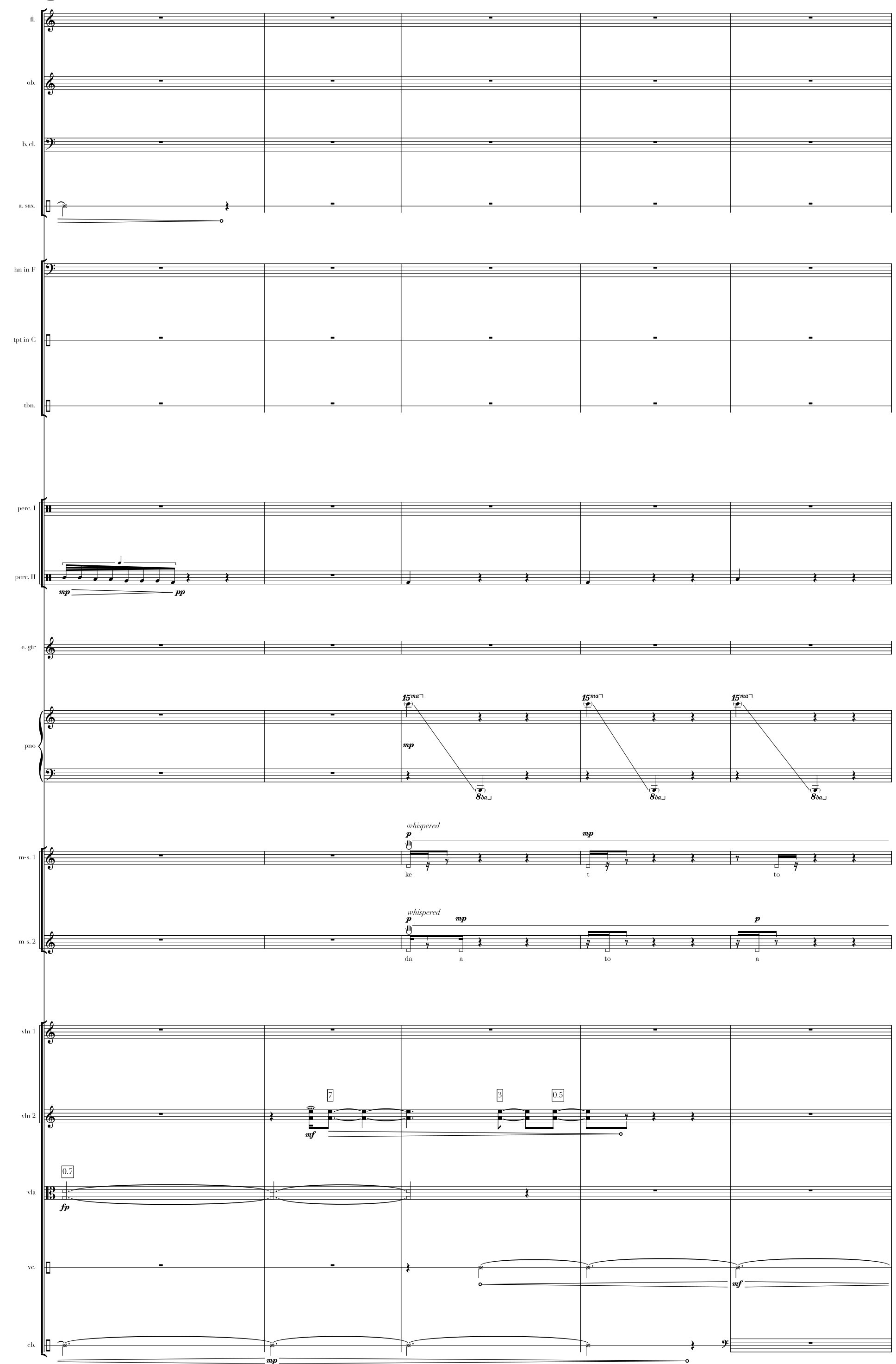

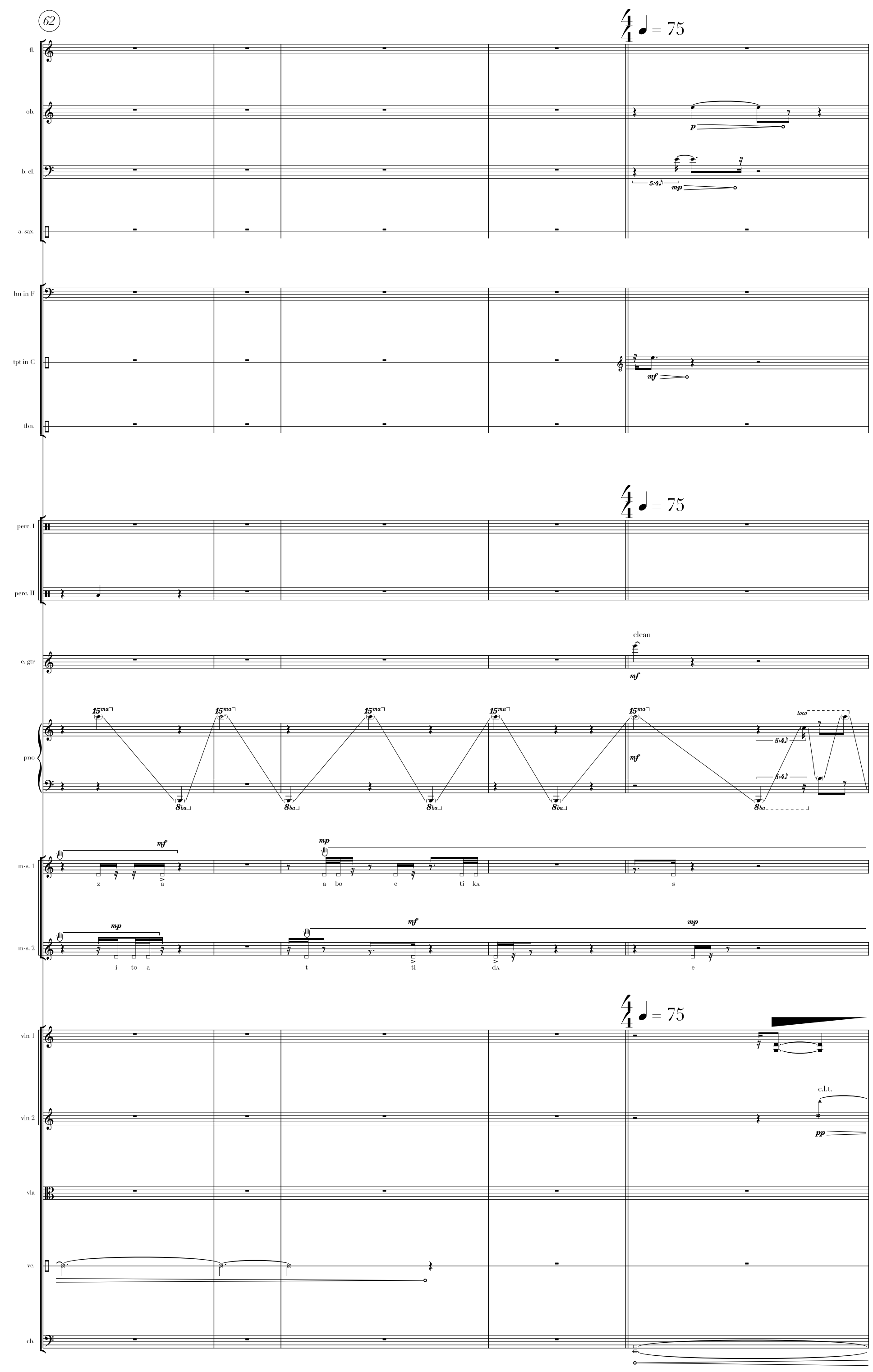

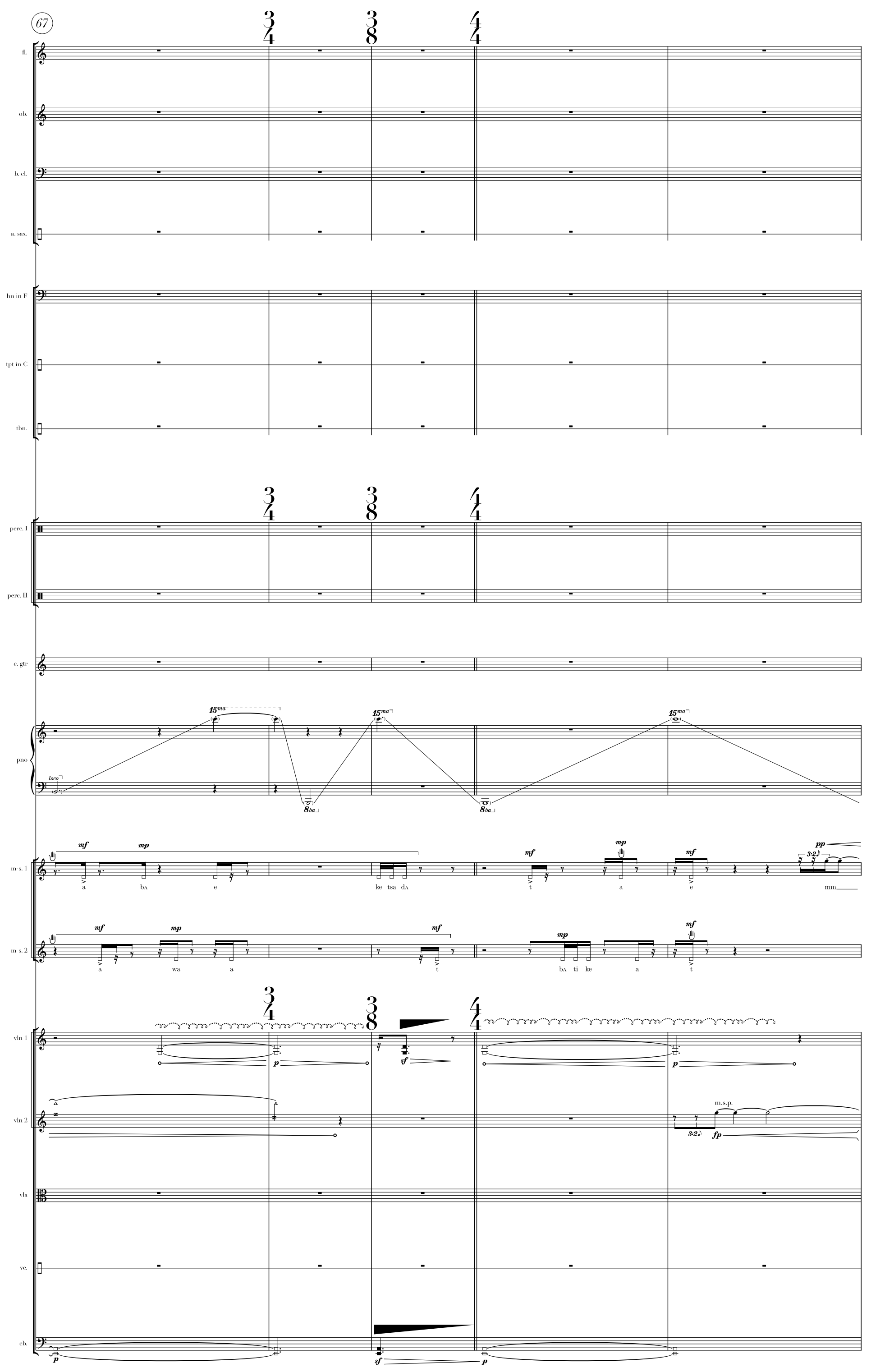

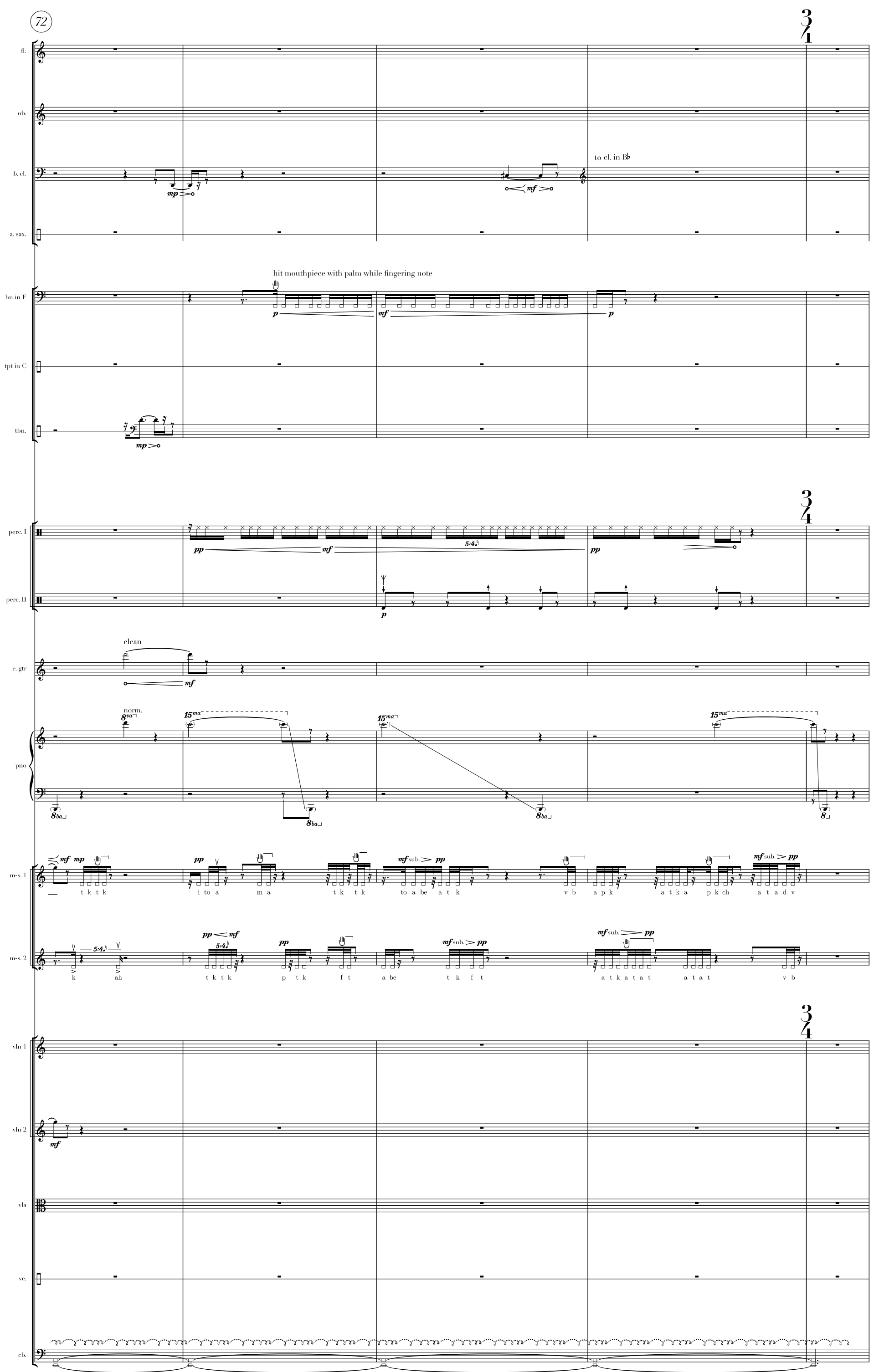

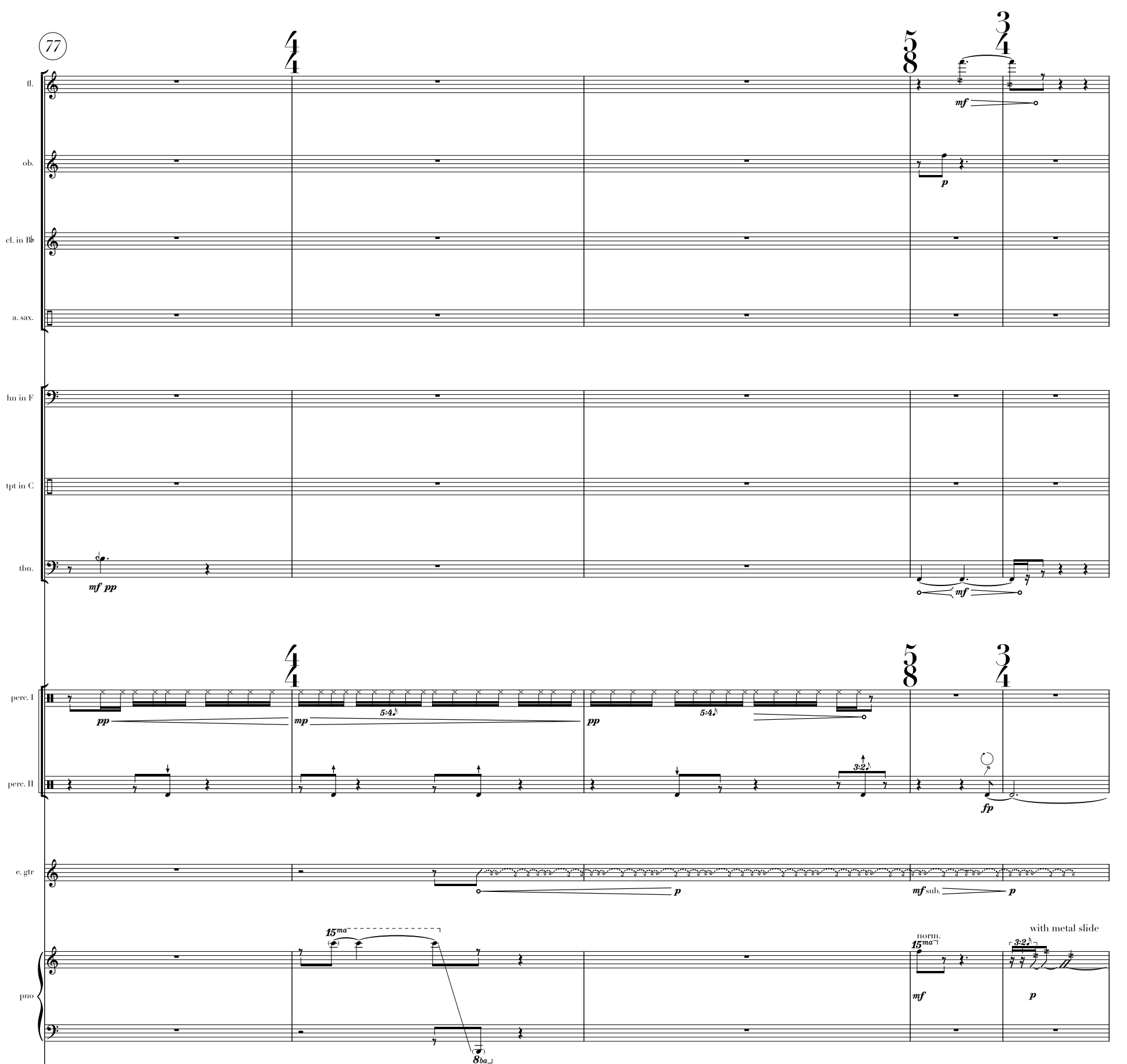

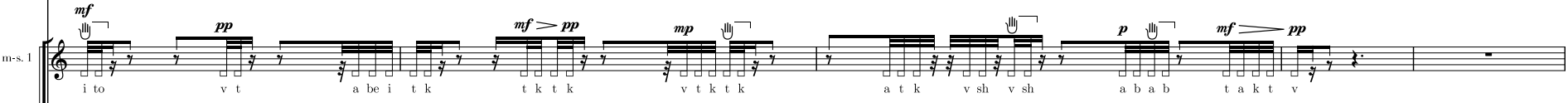
(2)

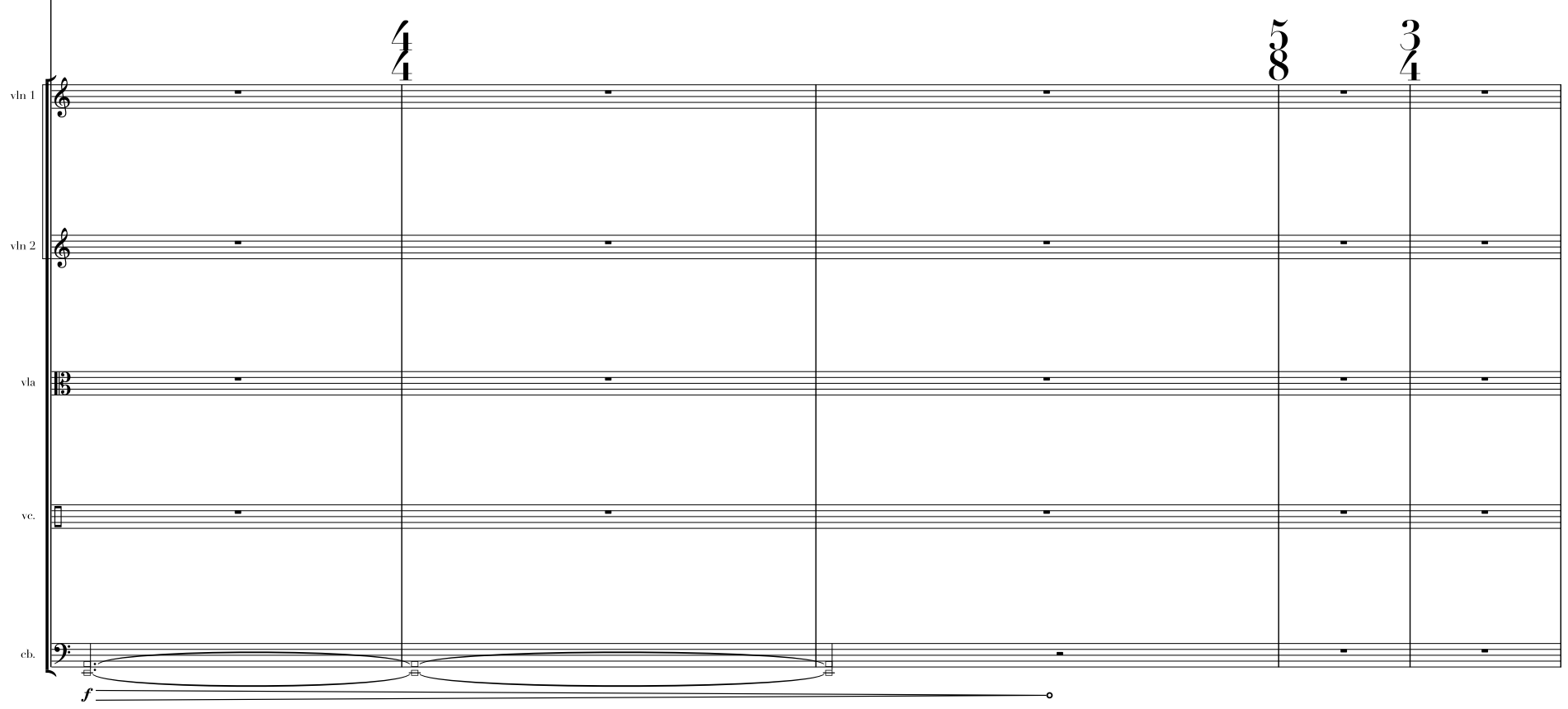



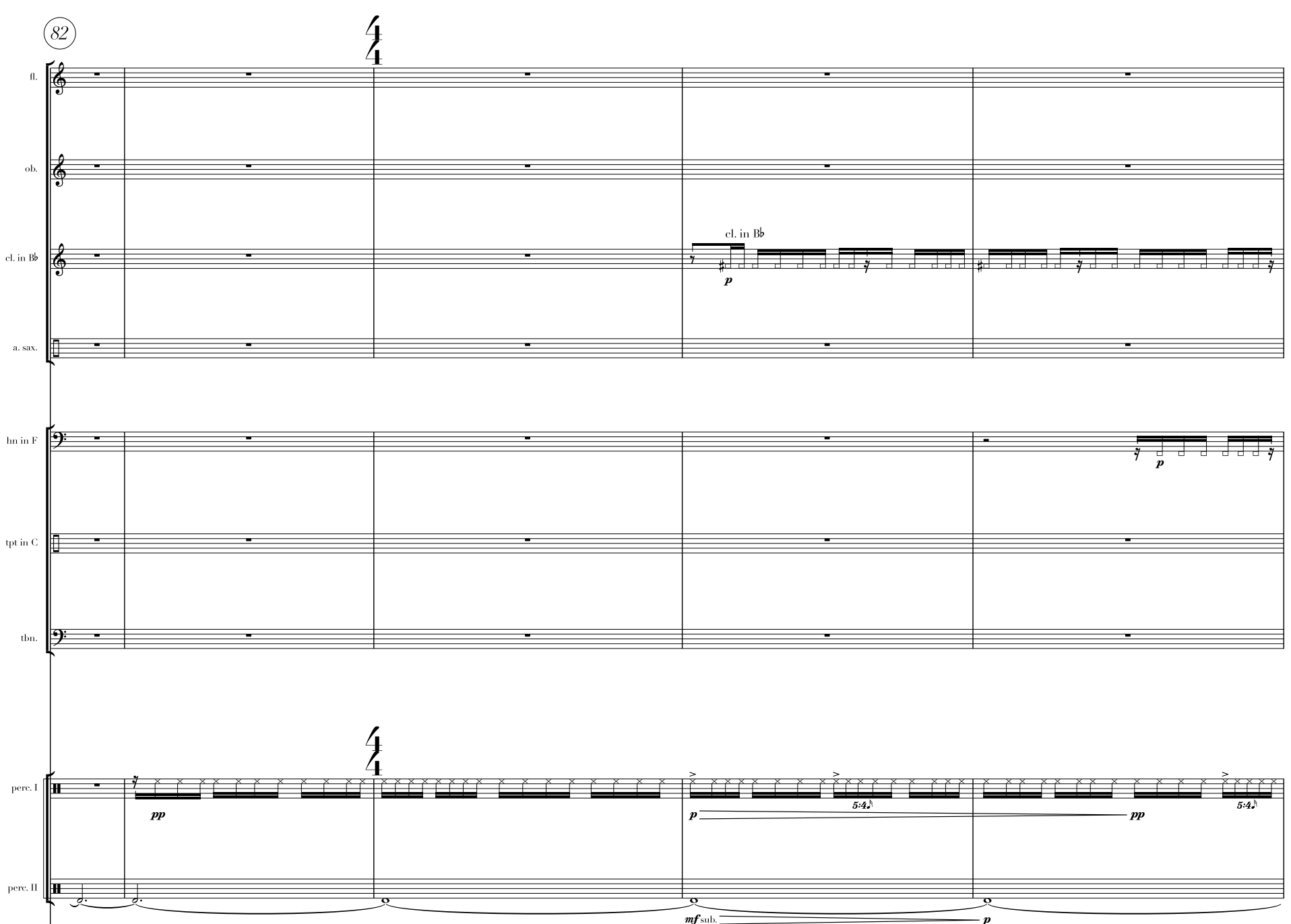

(2)

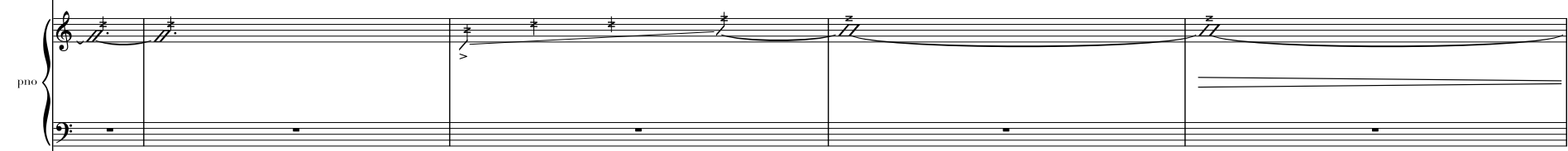

(2)

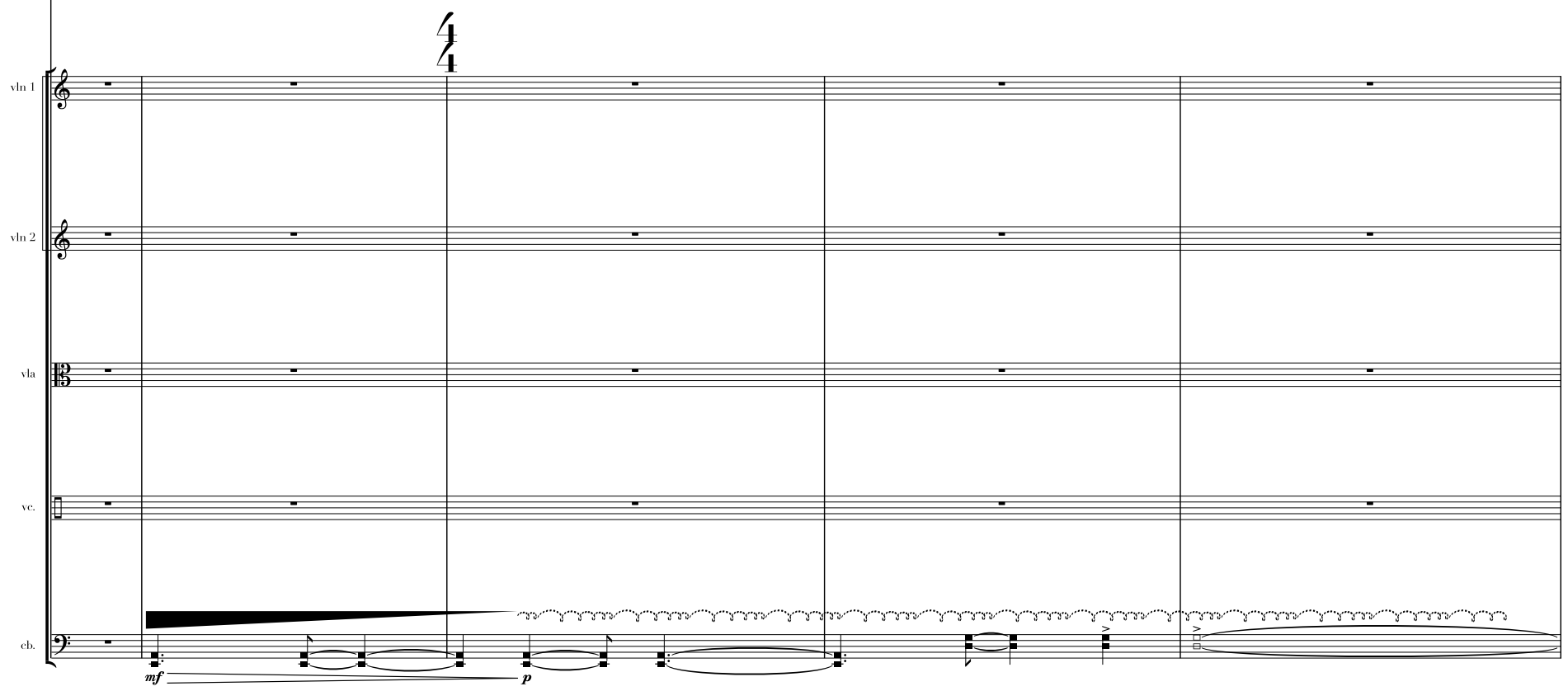



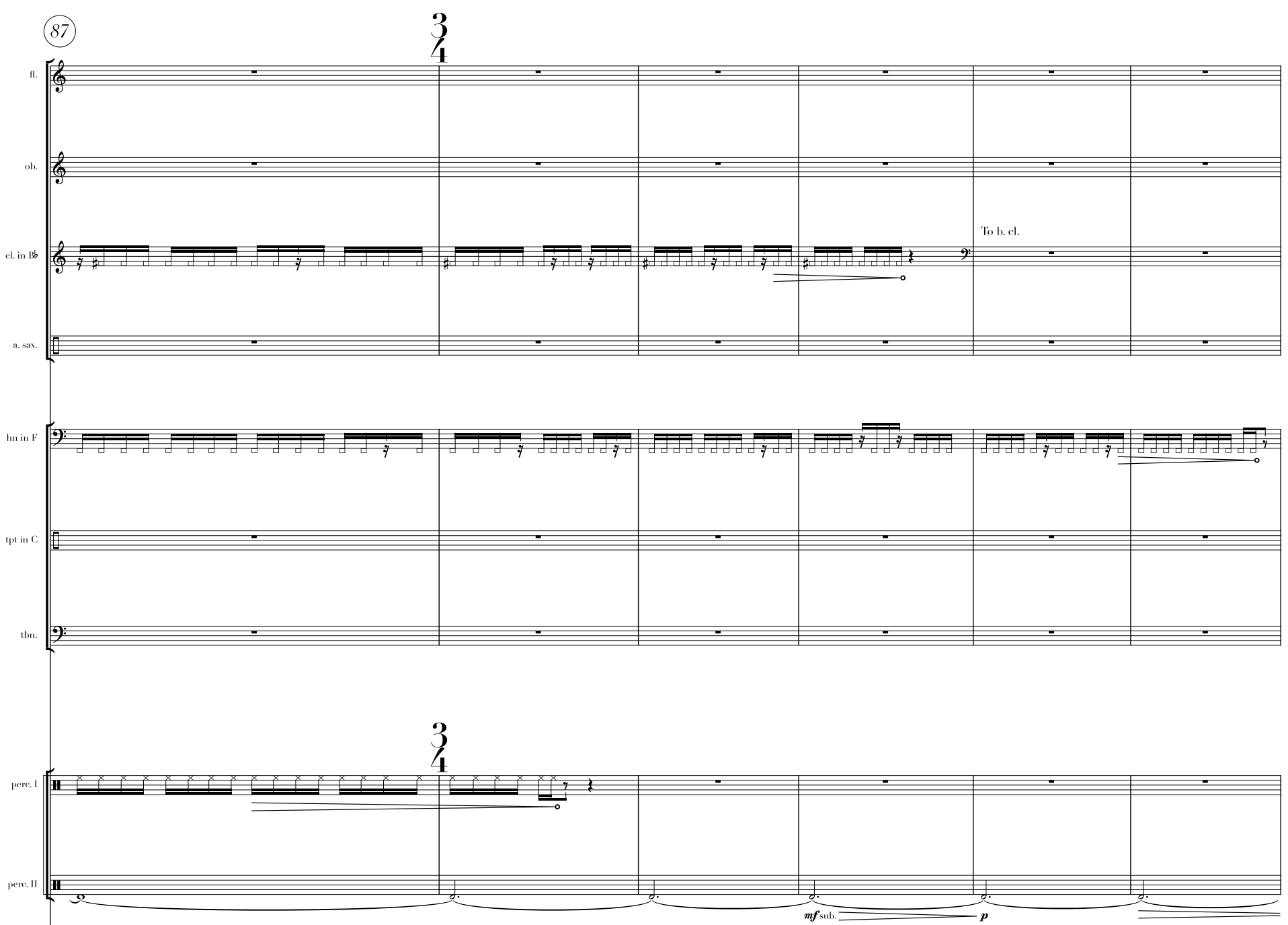

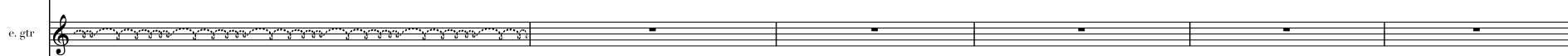
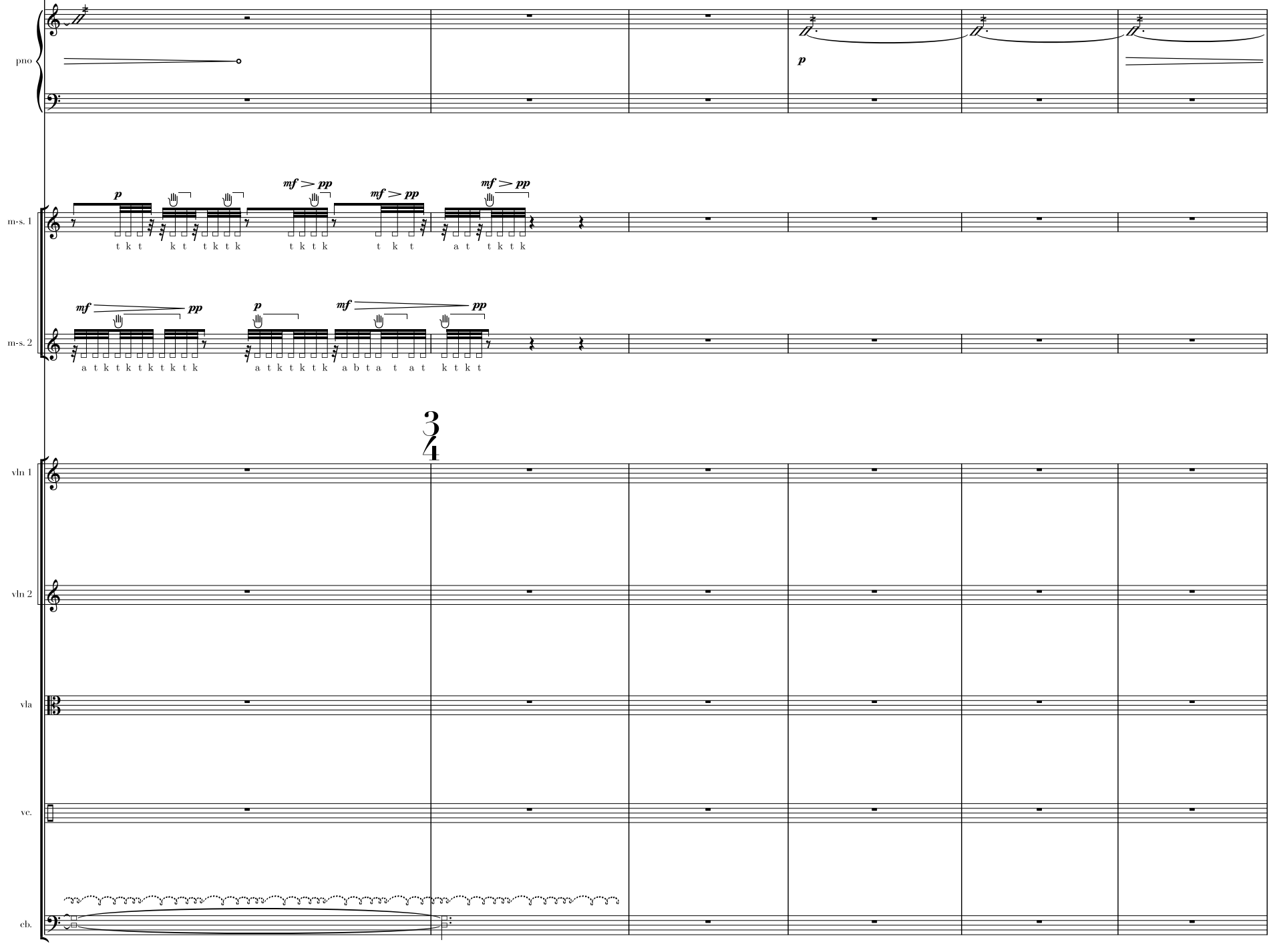
ii.

53
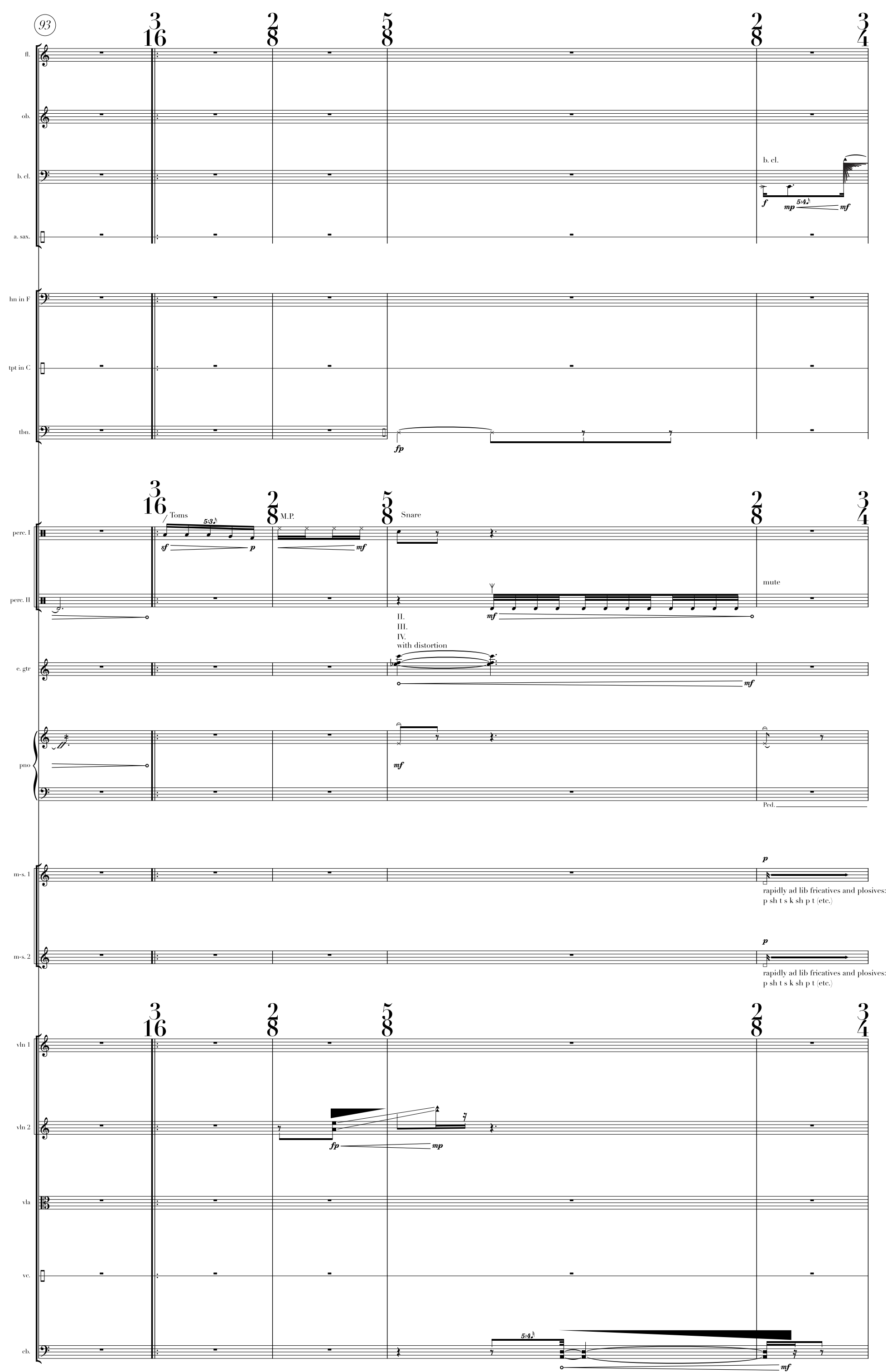


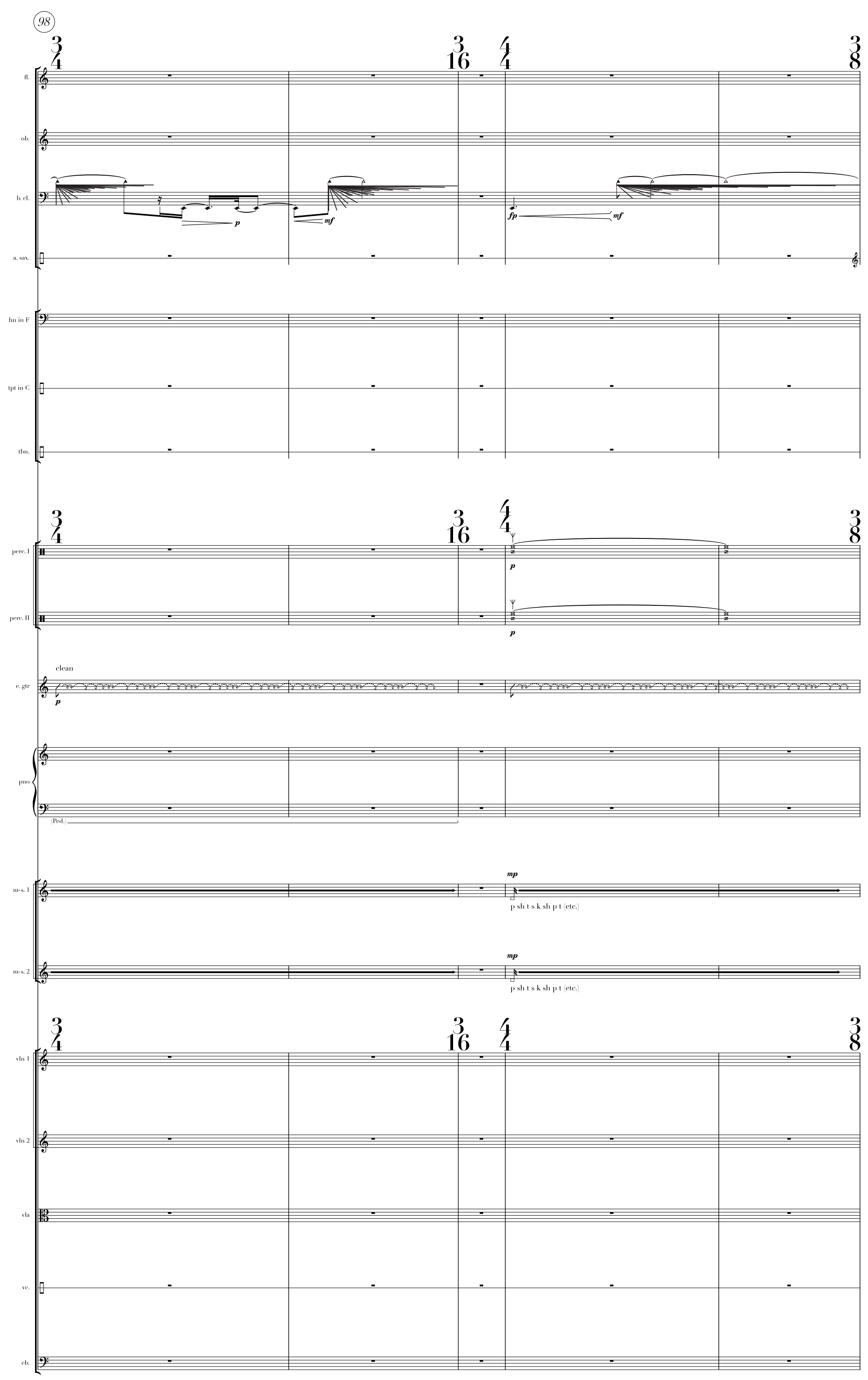

8

8 

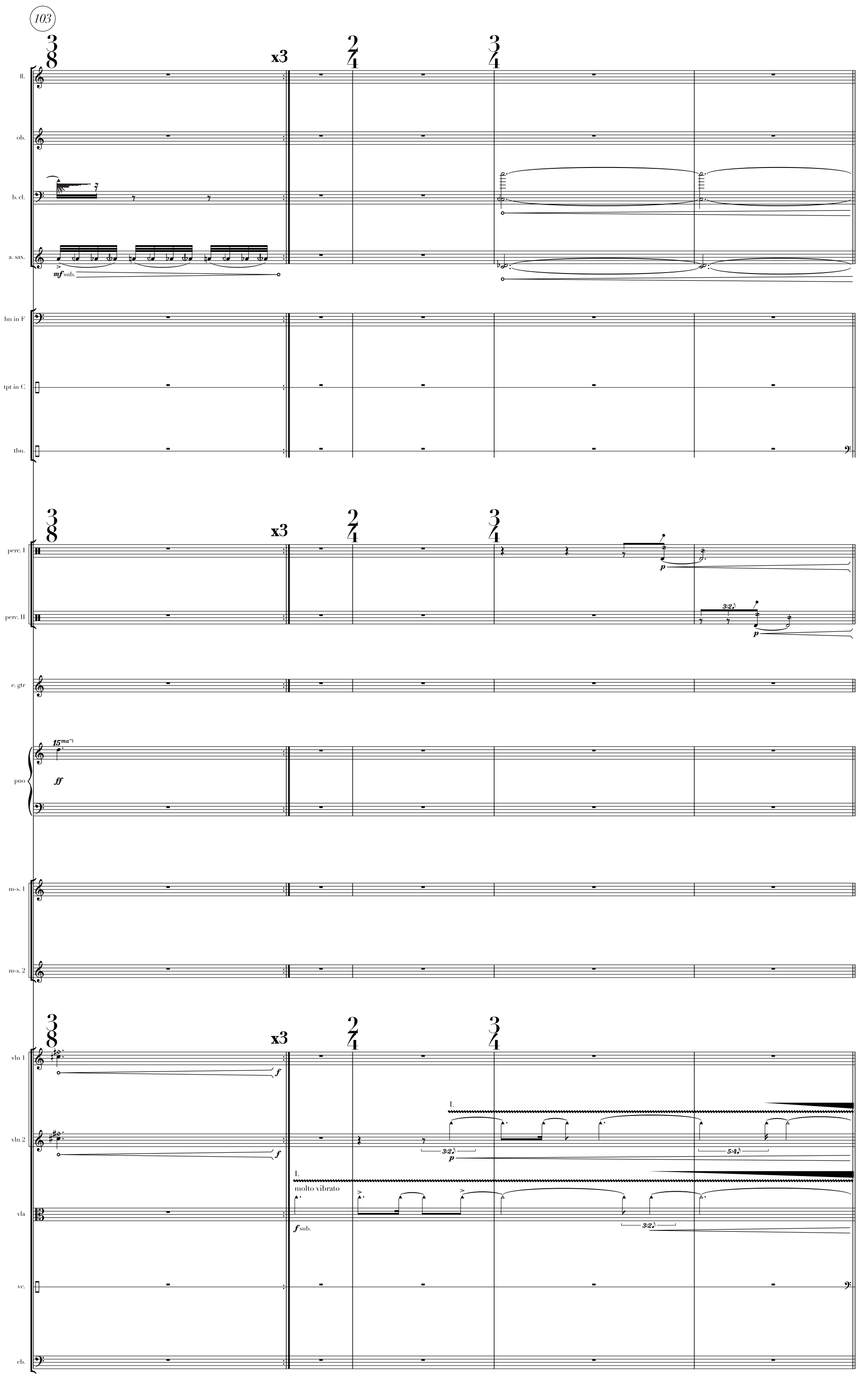


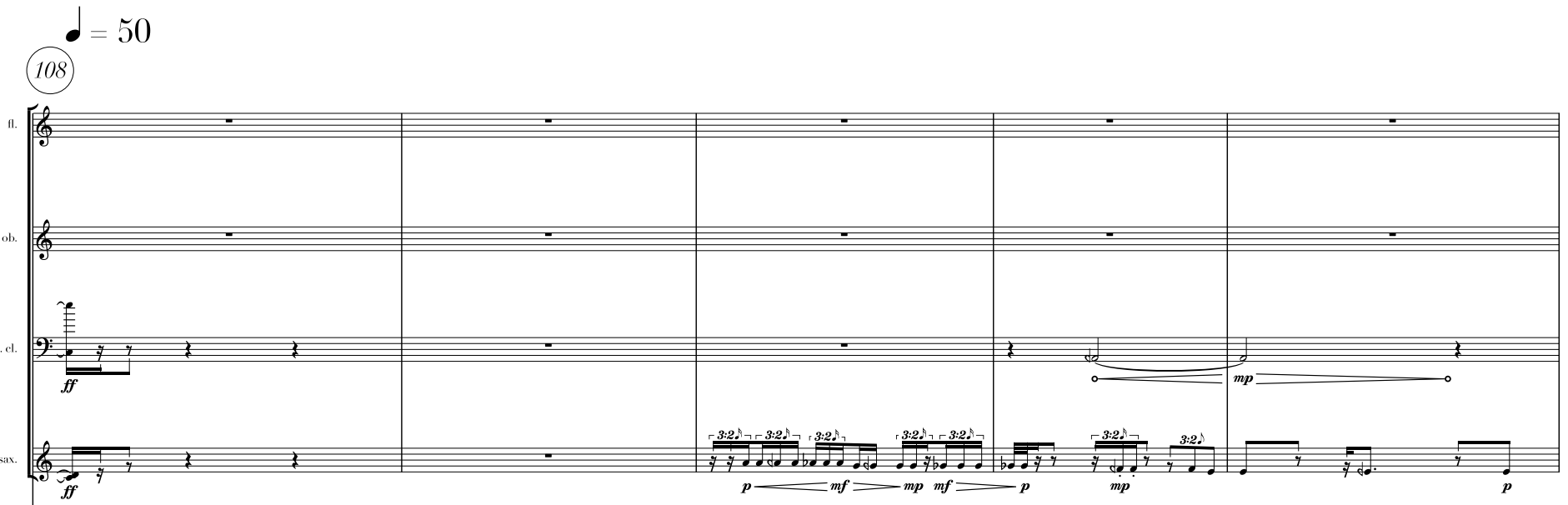

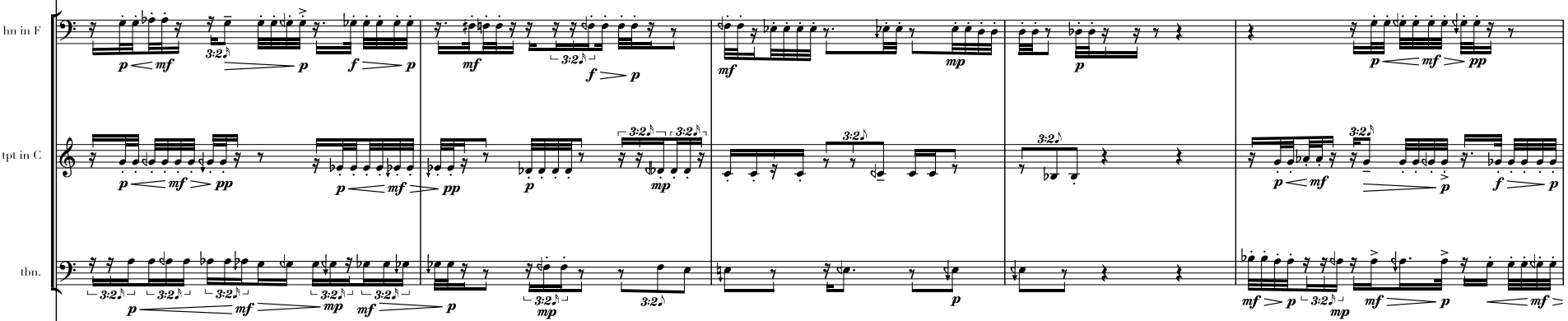
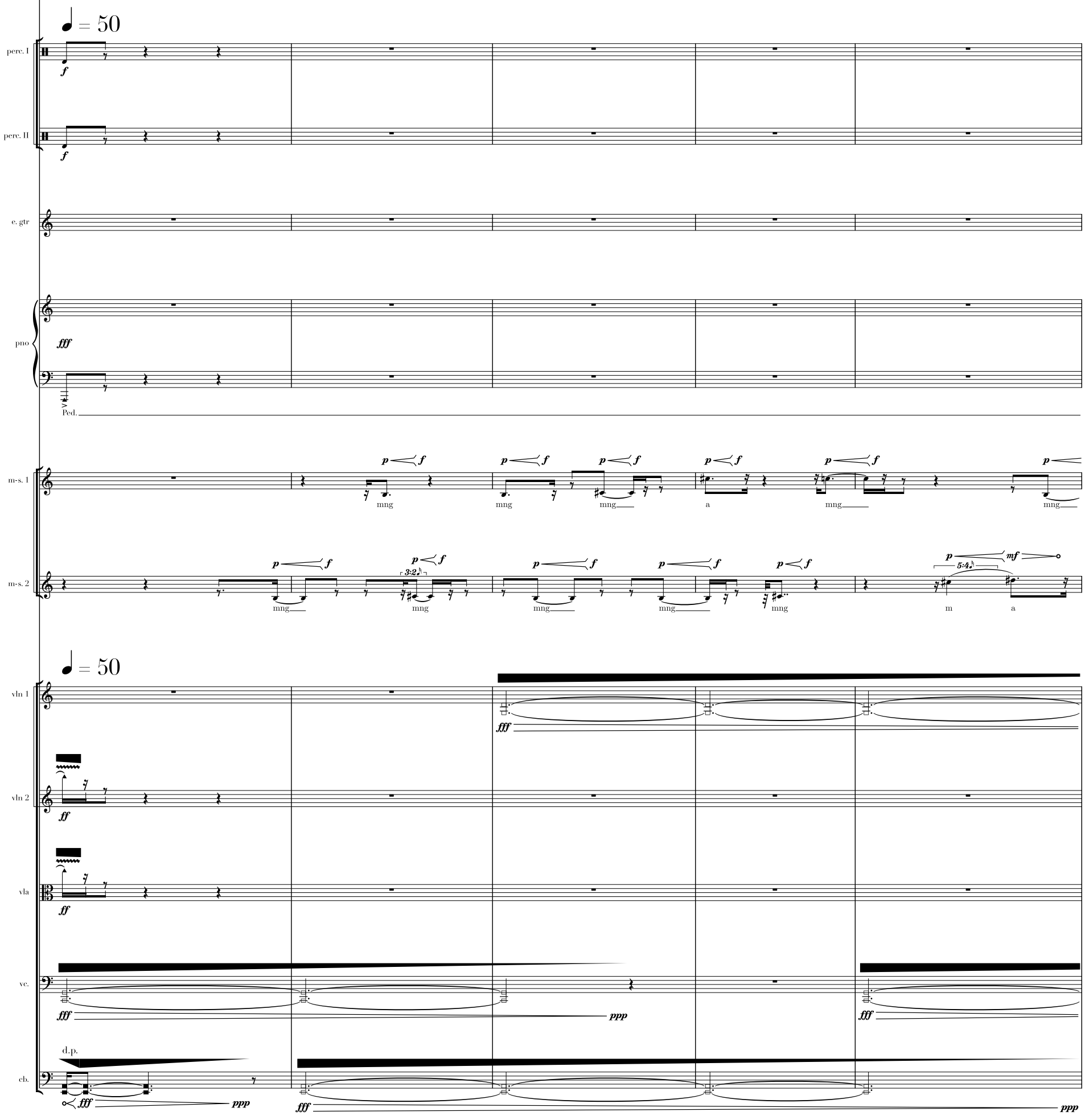

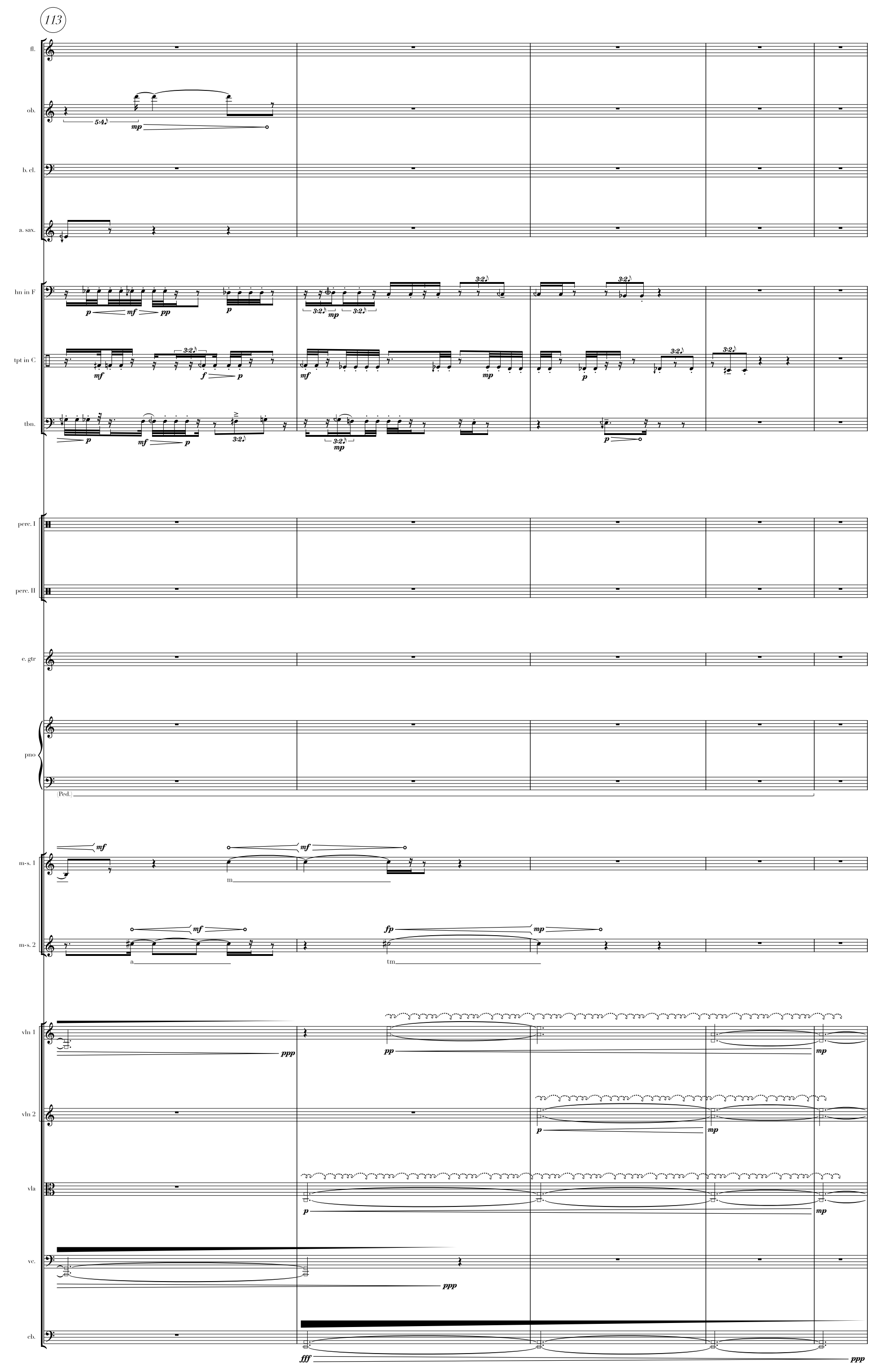

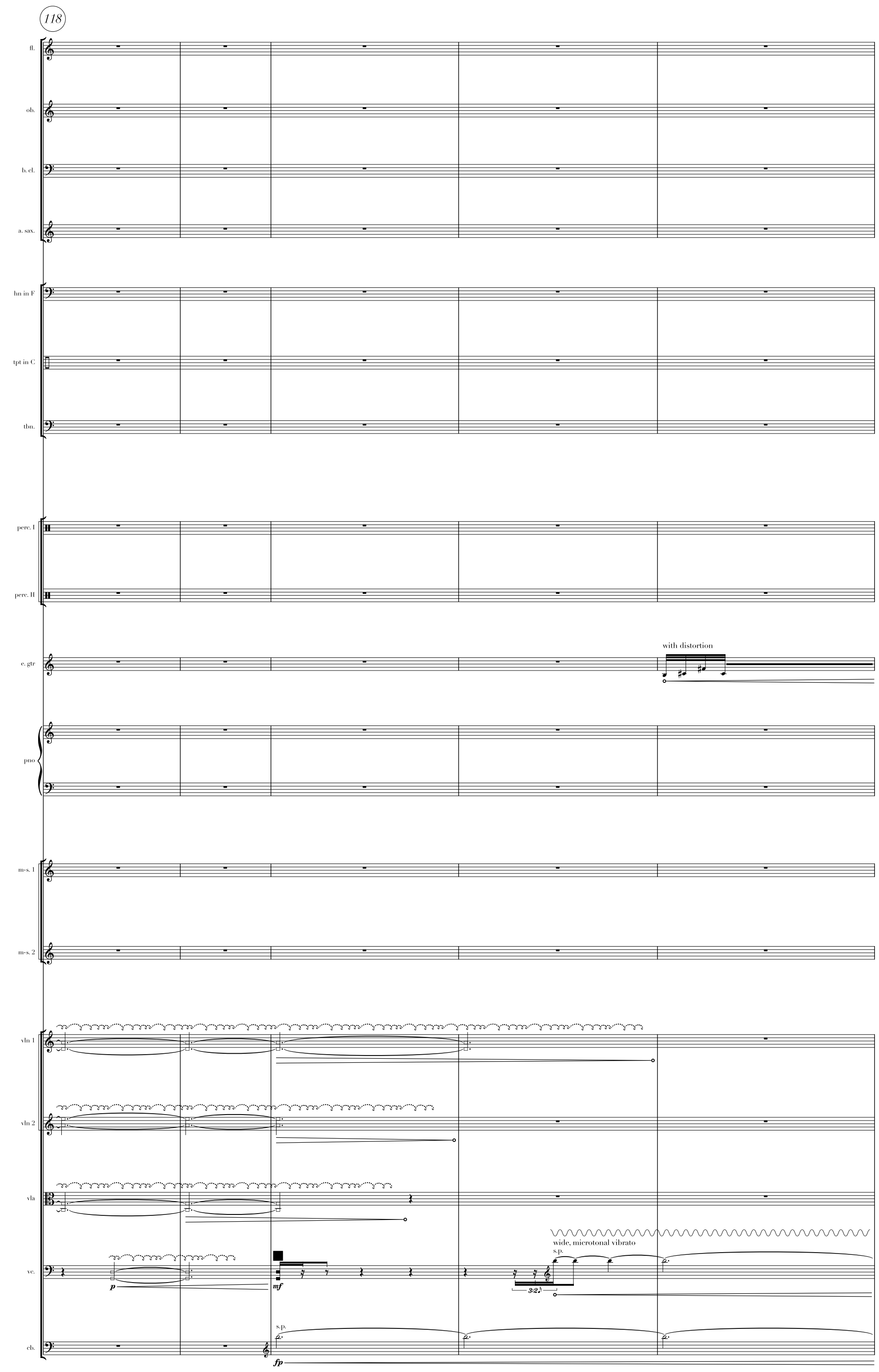
(123)
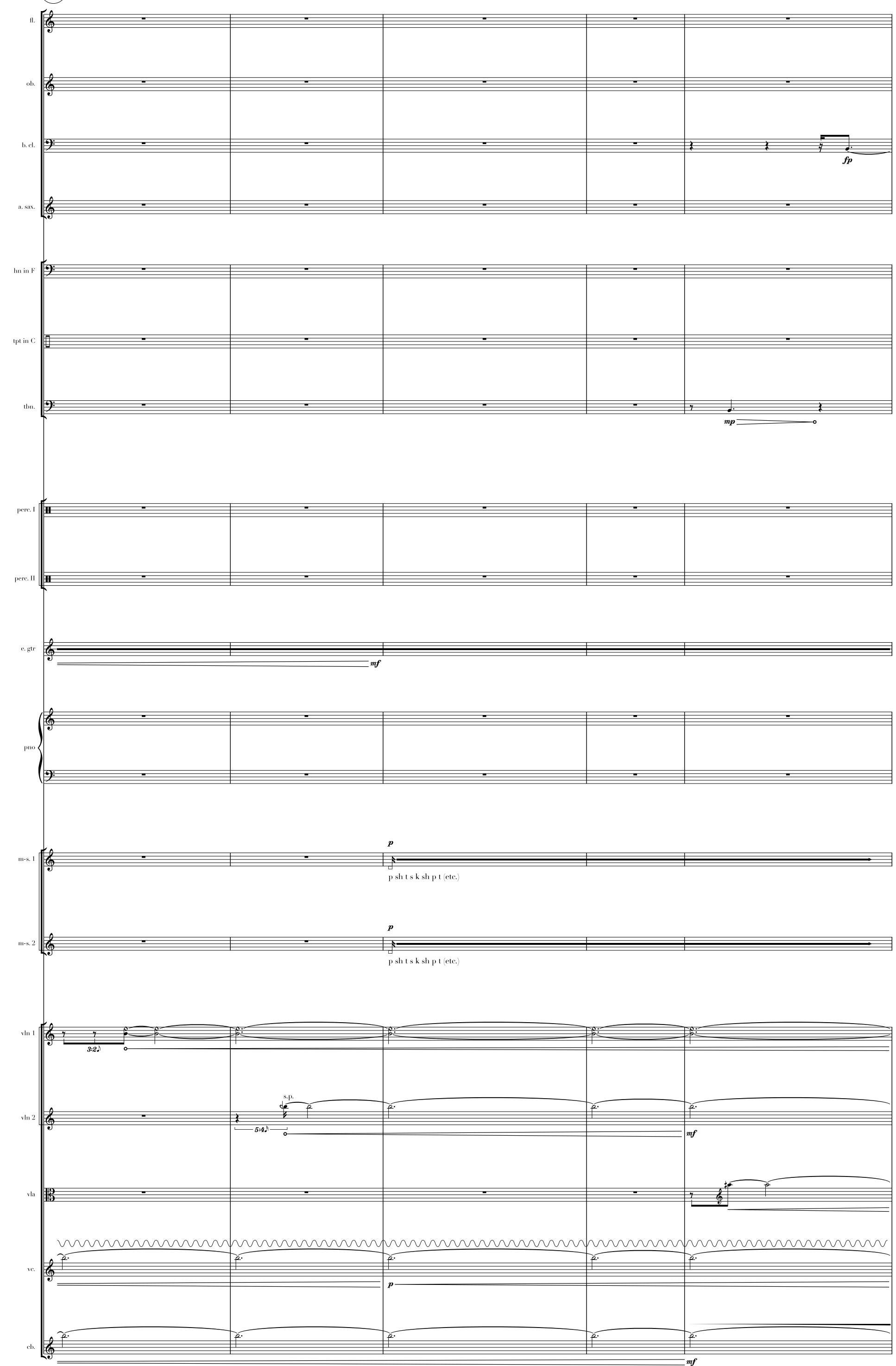


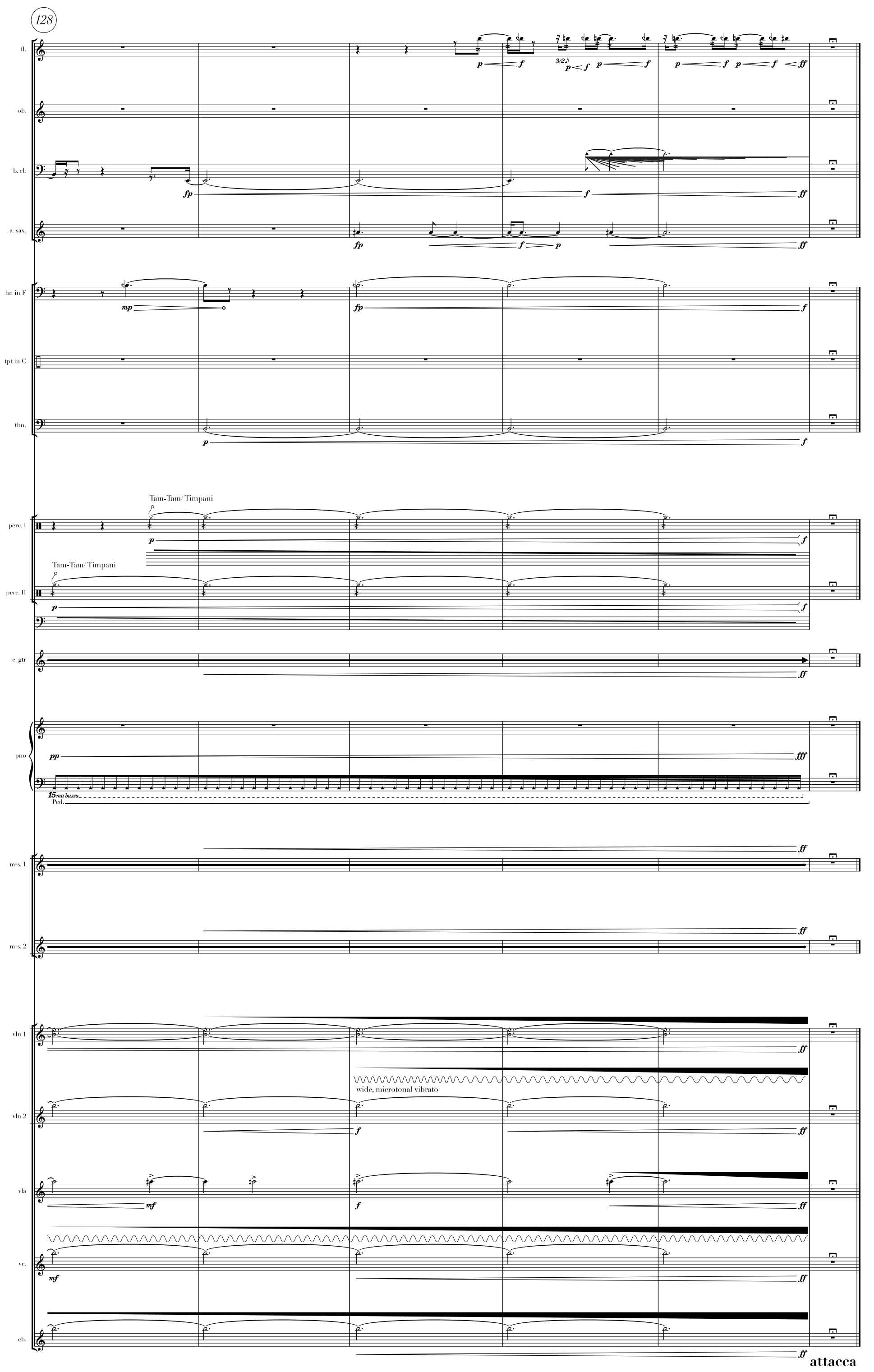


iii.
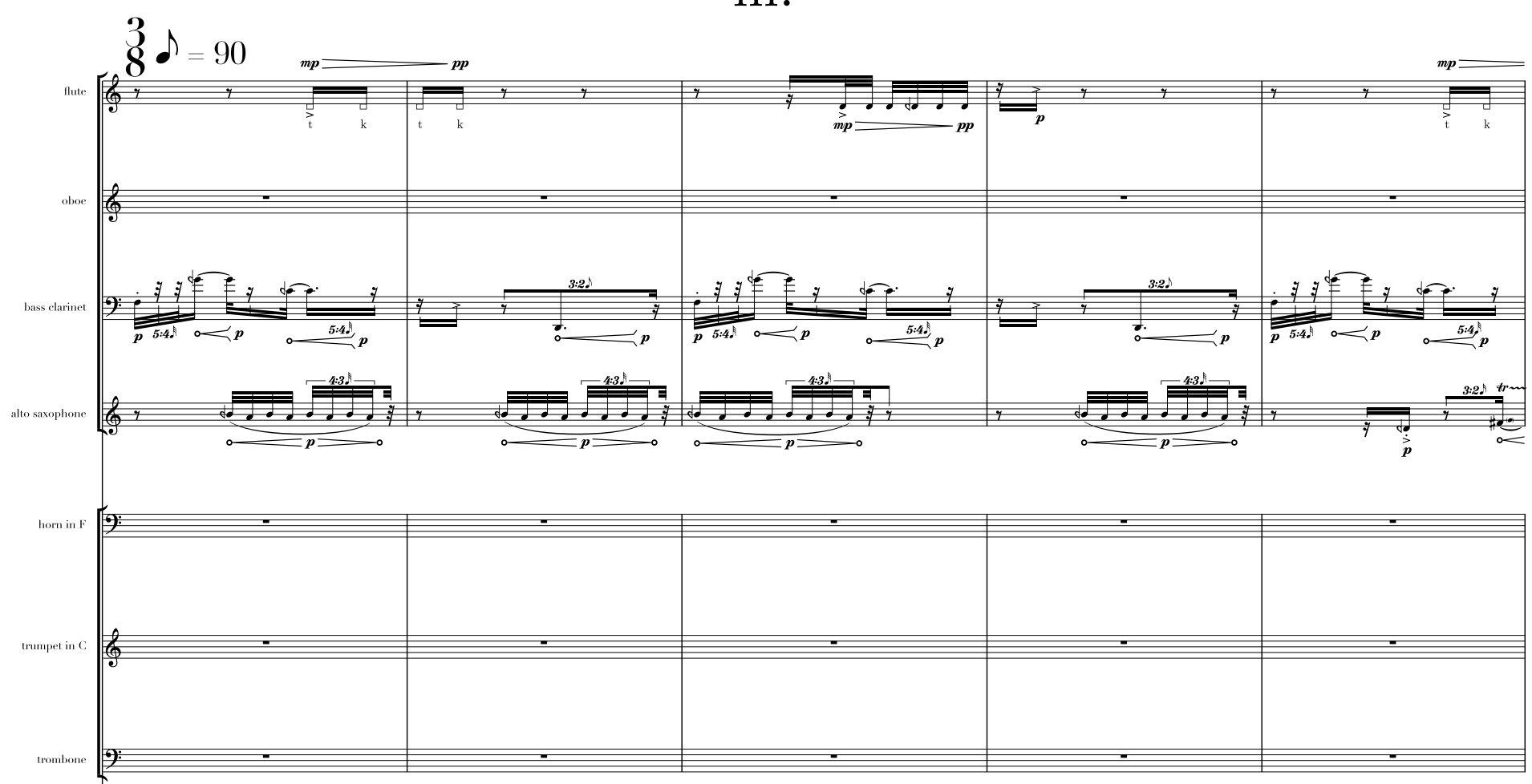

$8^{d}=90$

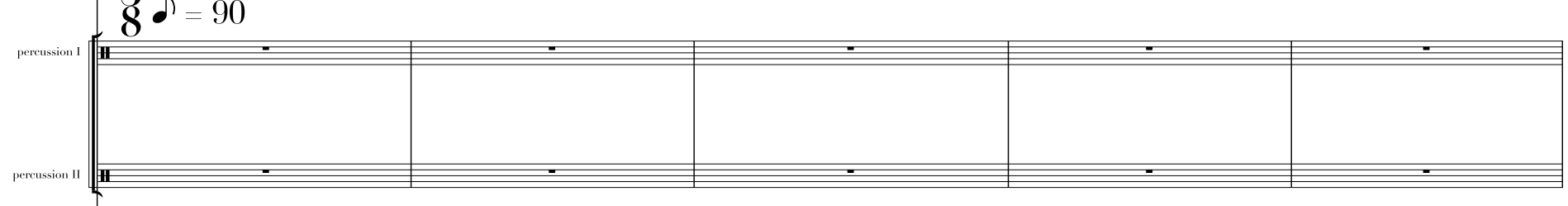

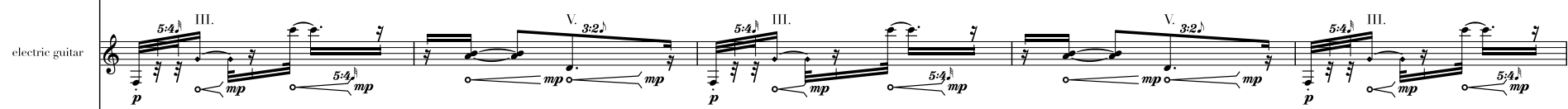

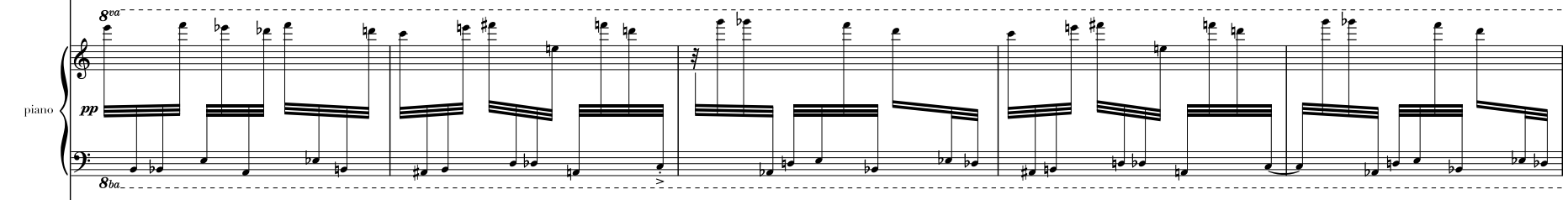
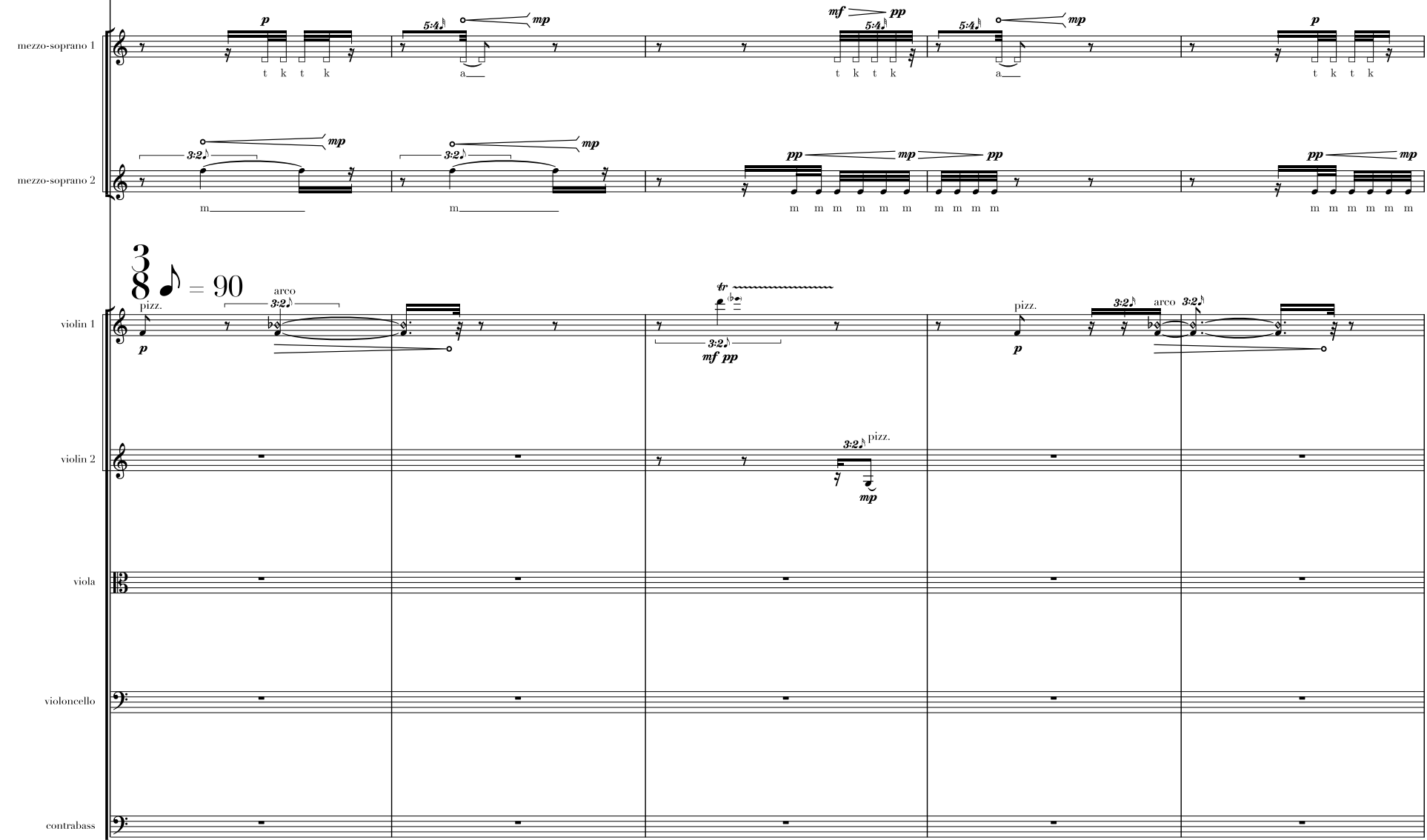

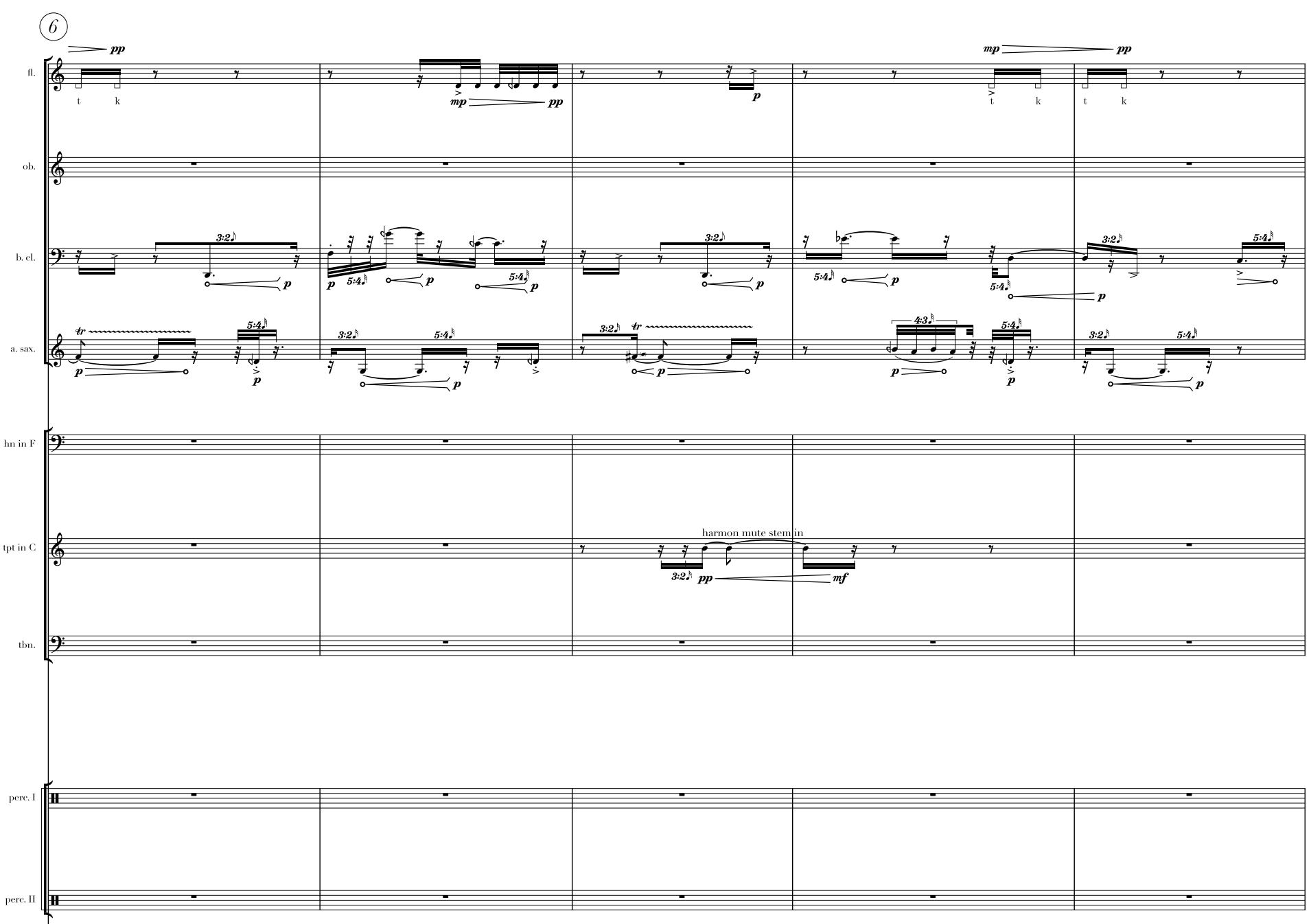

(at)
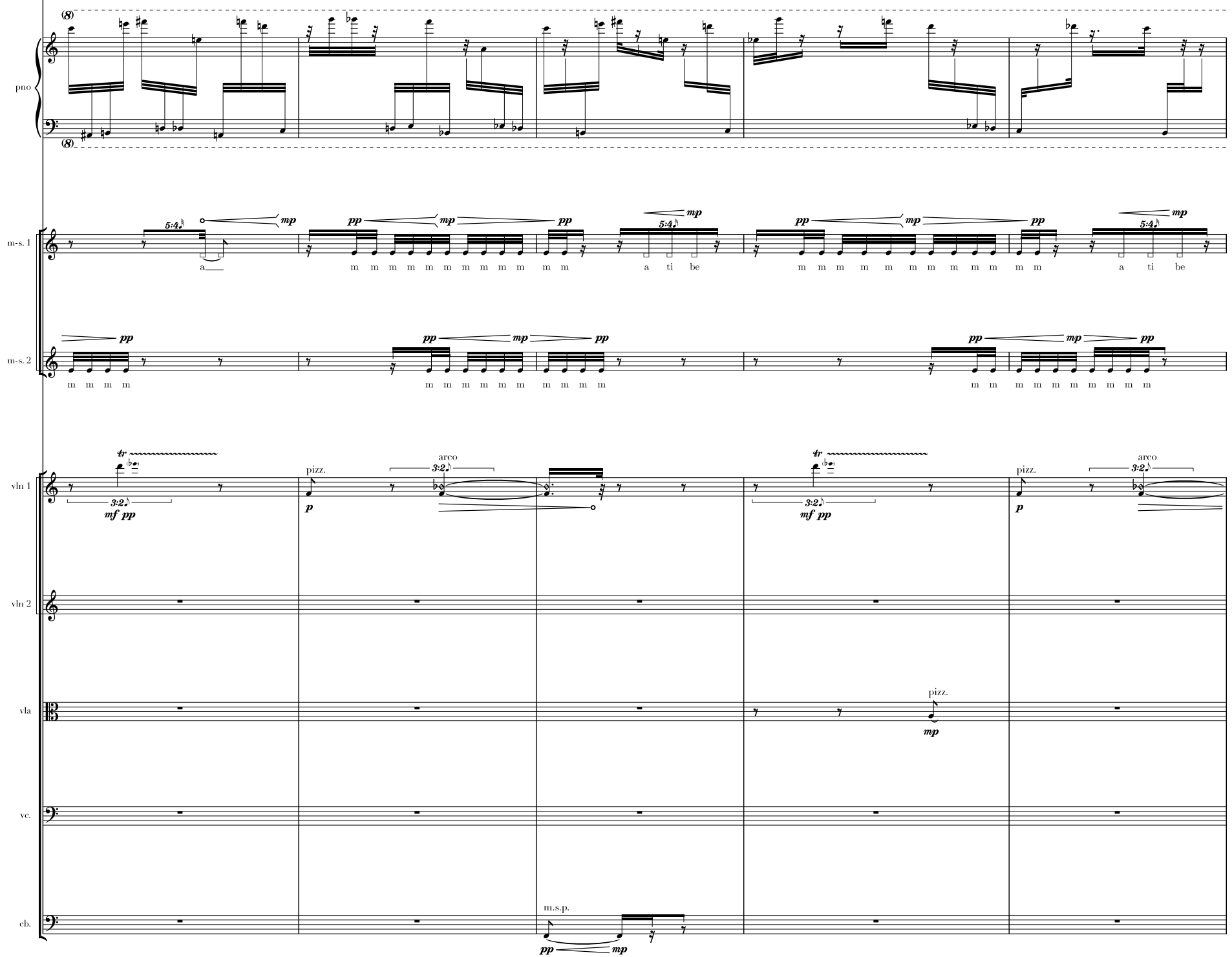

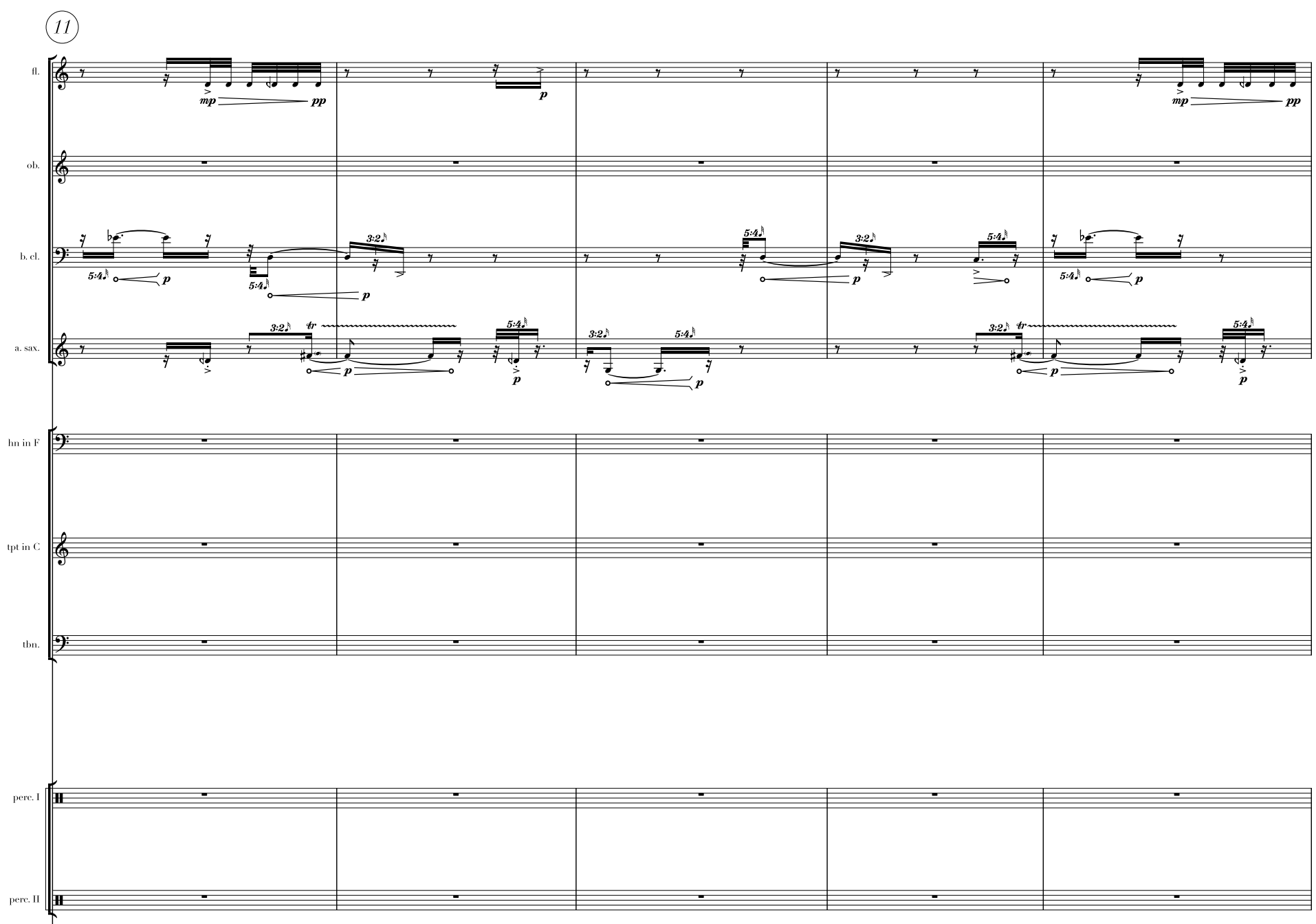

(5)

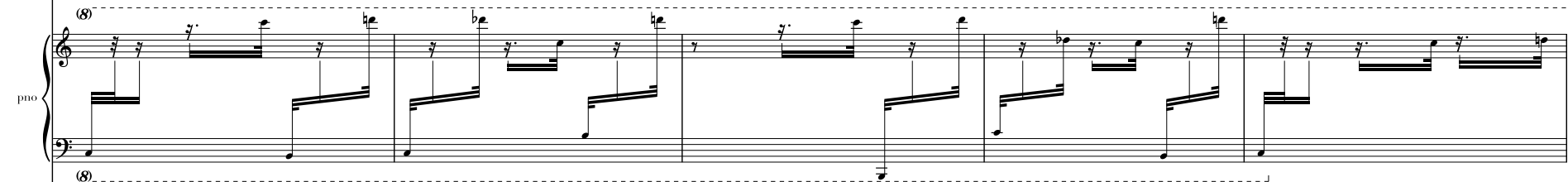
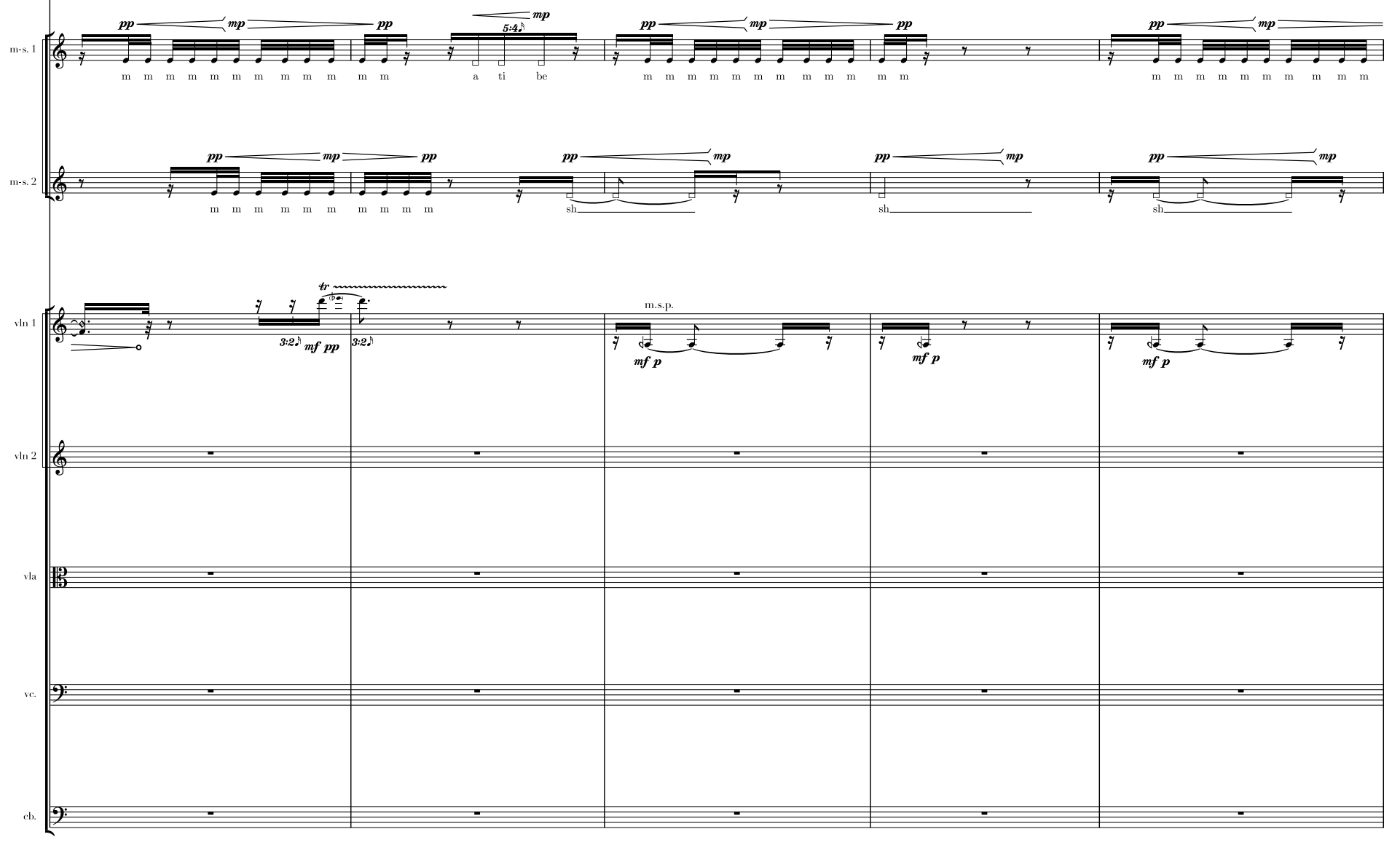

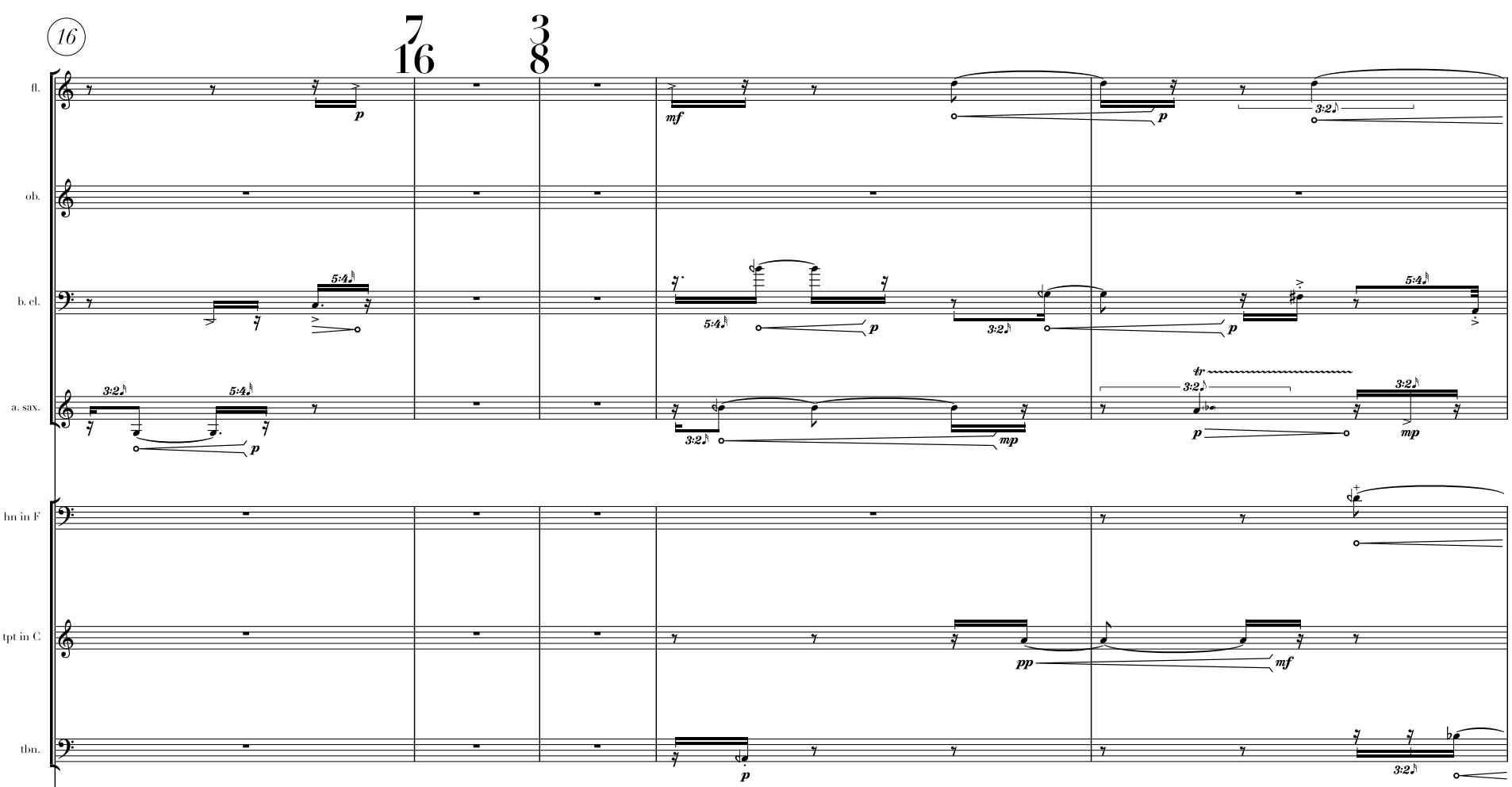

$\begin{array}{ll}7 & 3\end{array}$
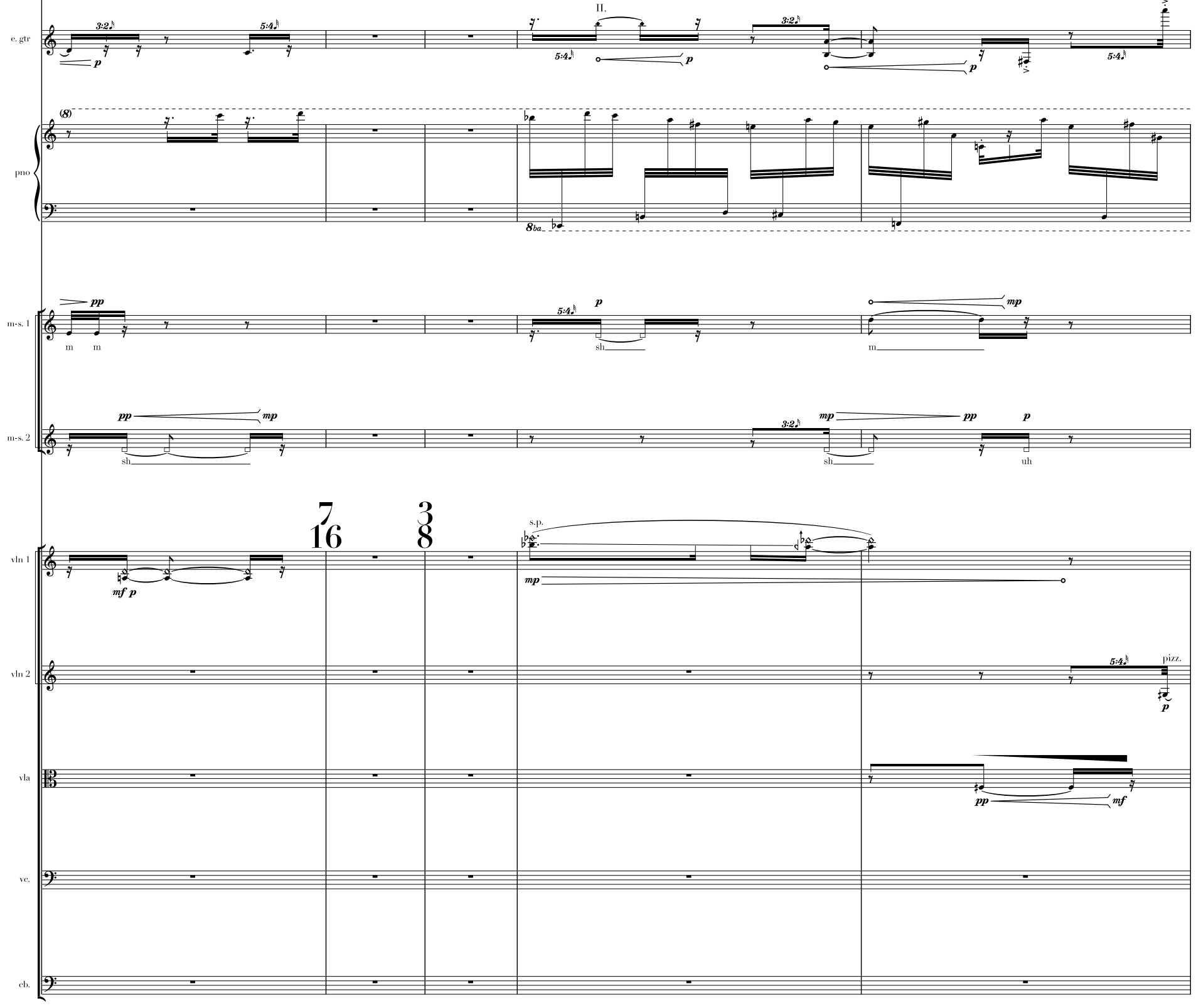
(21)
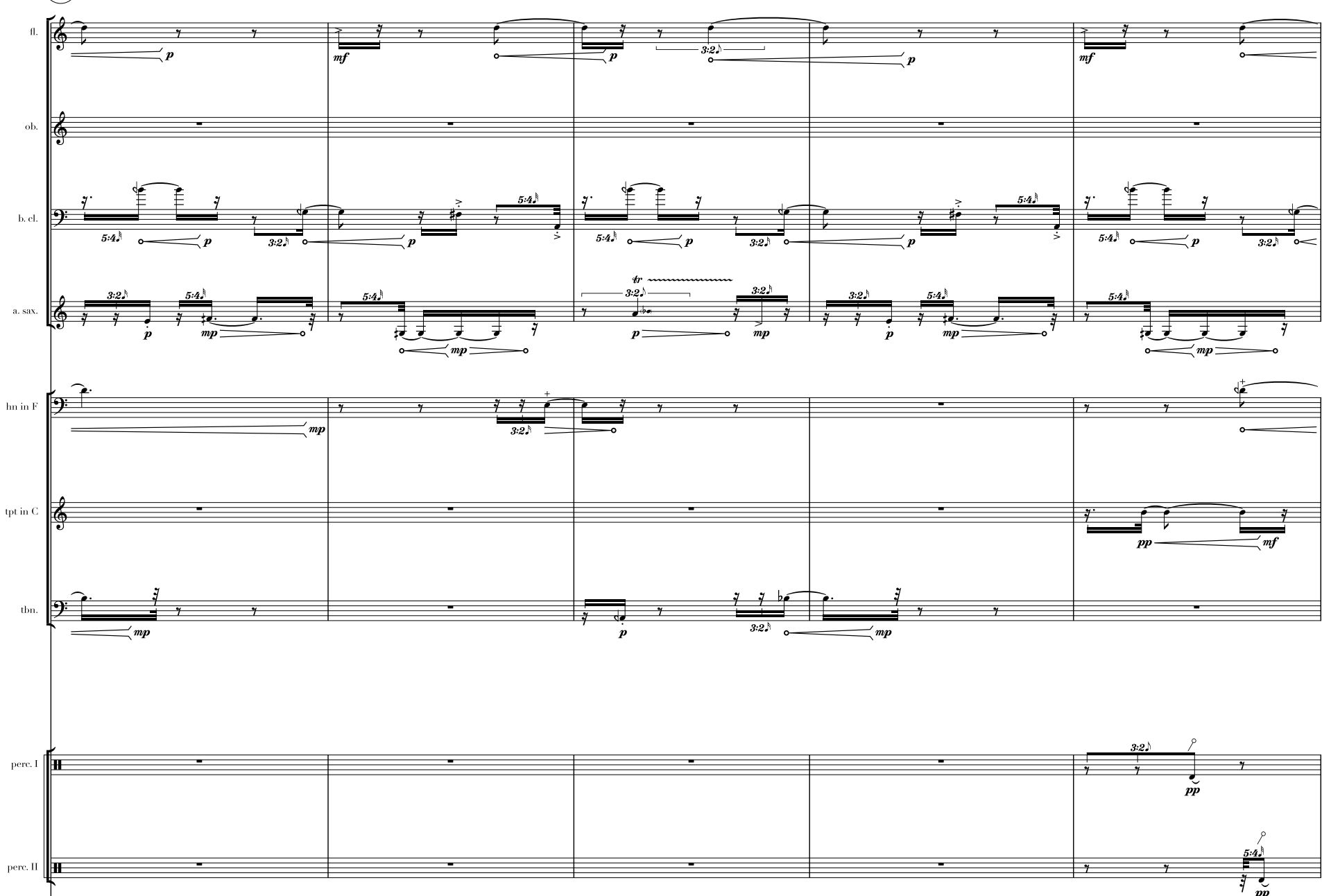

(and

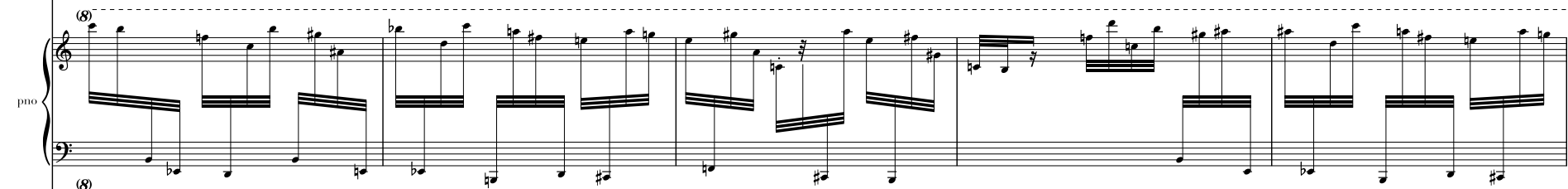
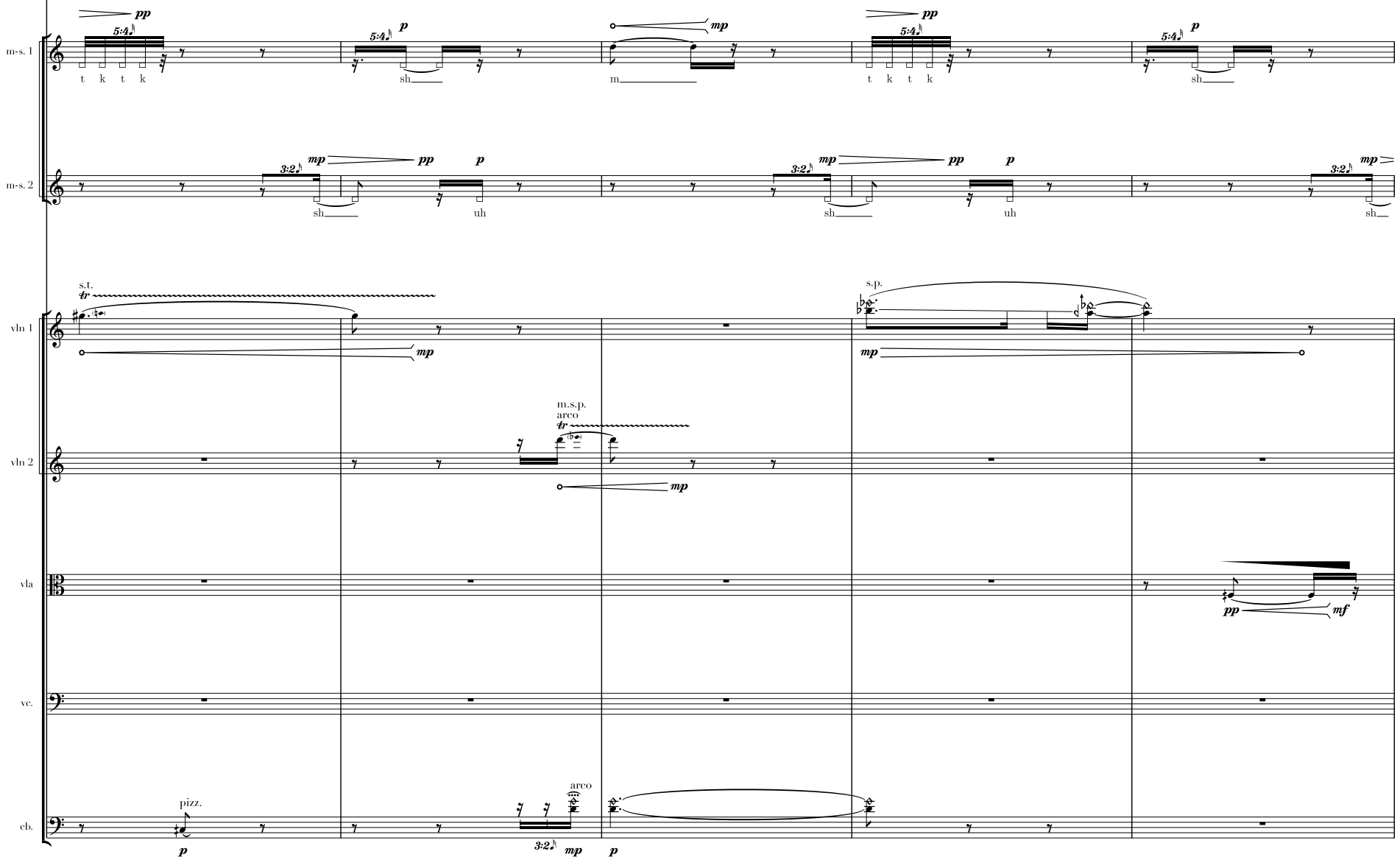
(26)
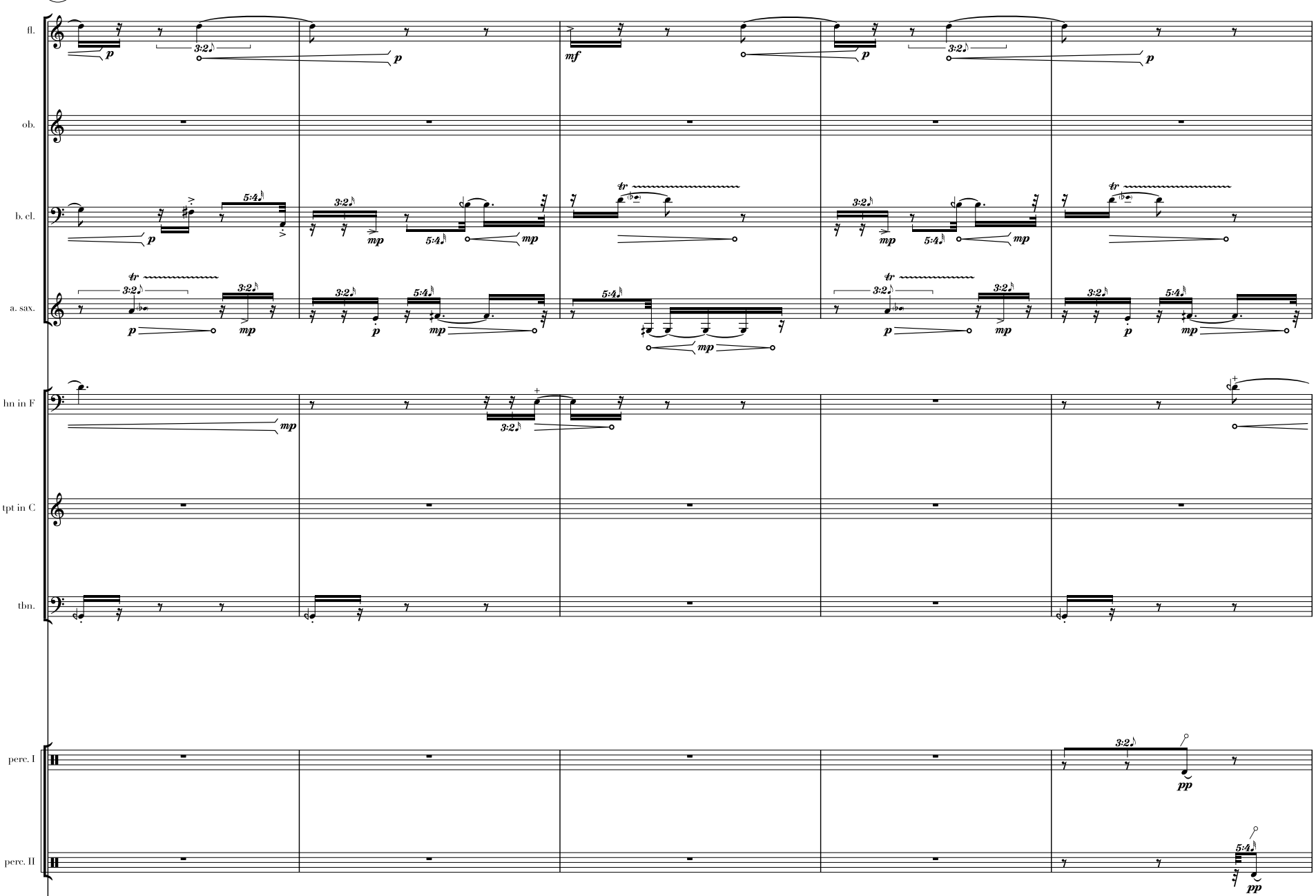

(6)

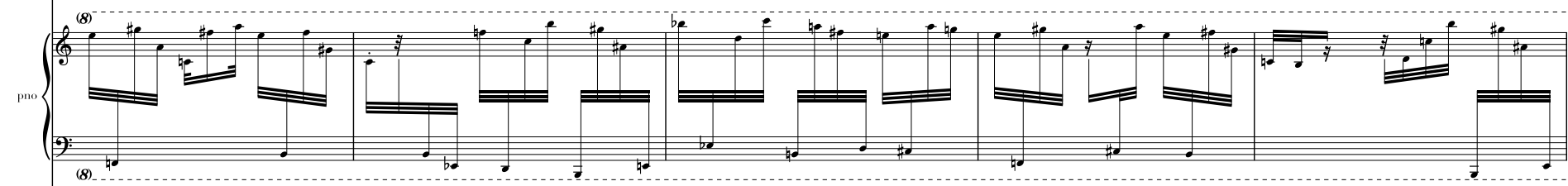
(n)

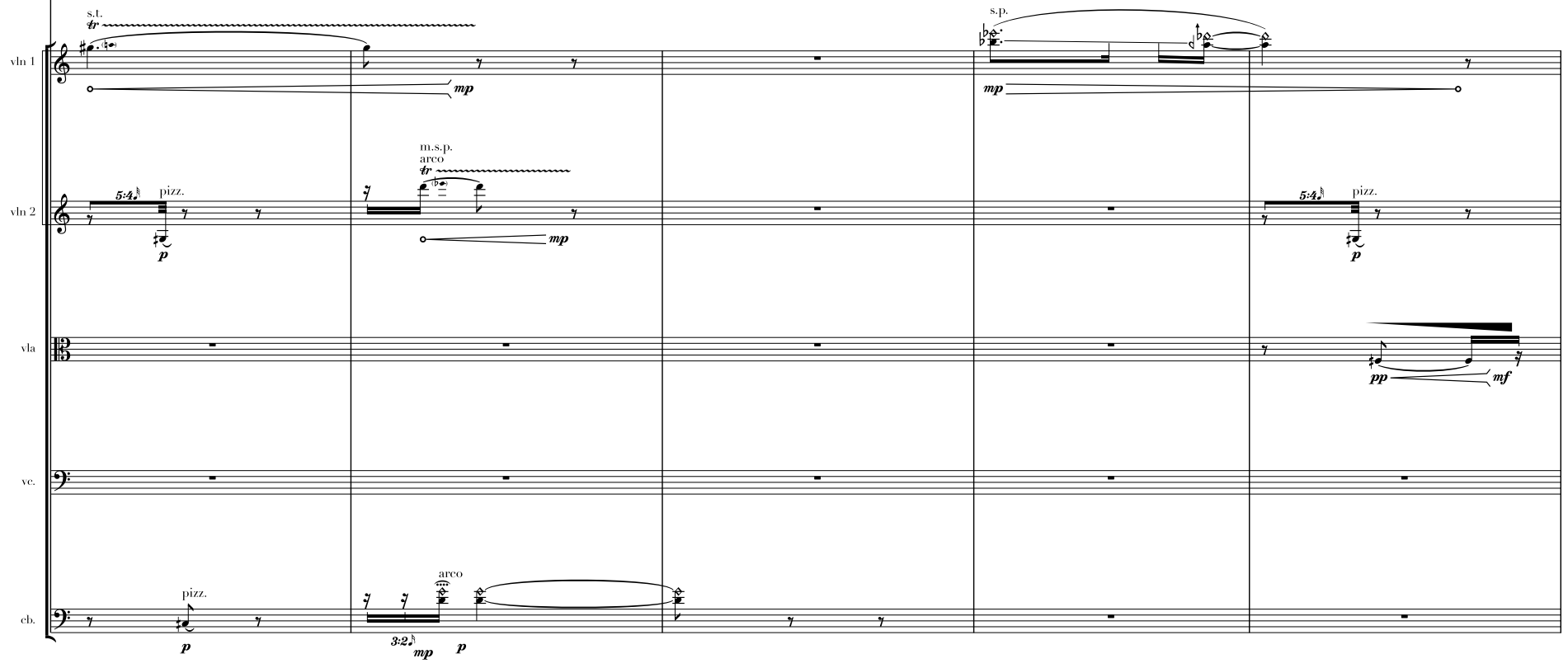



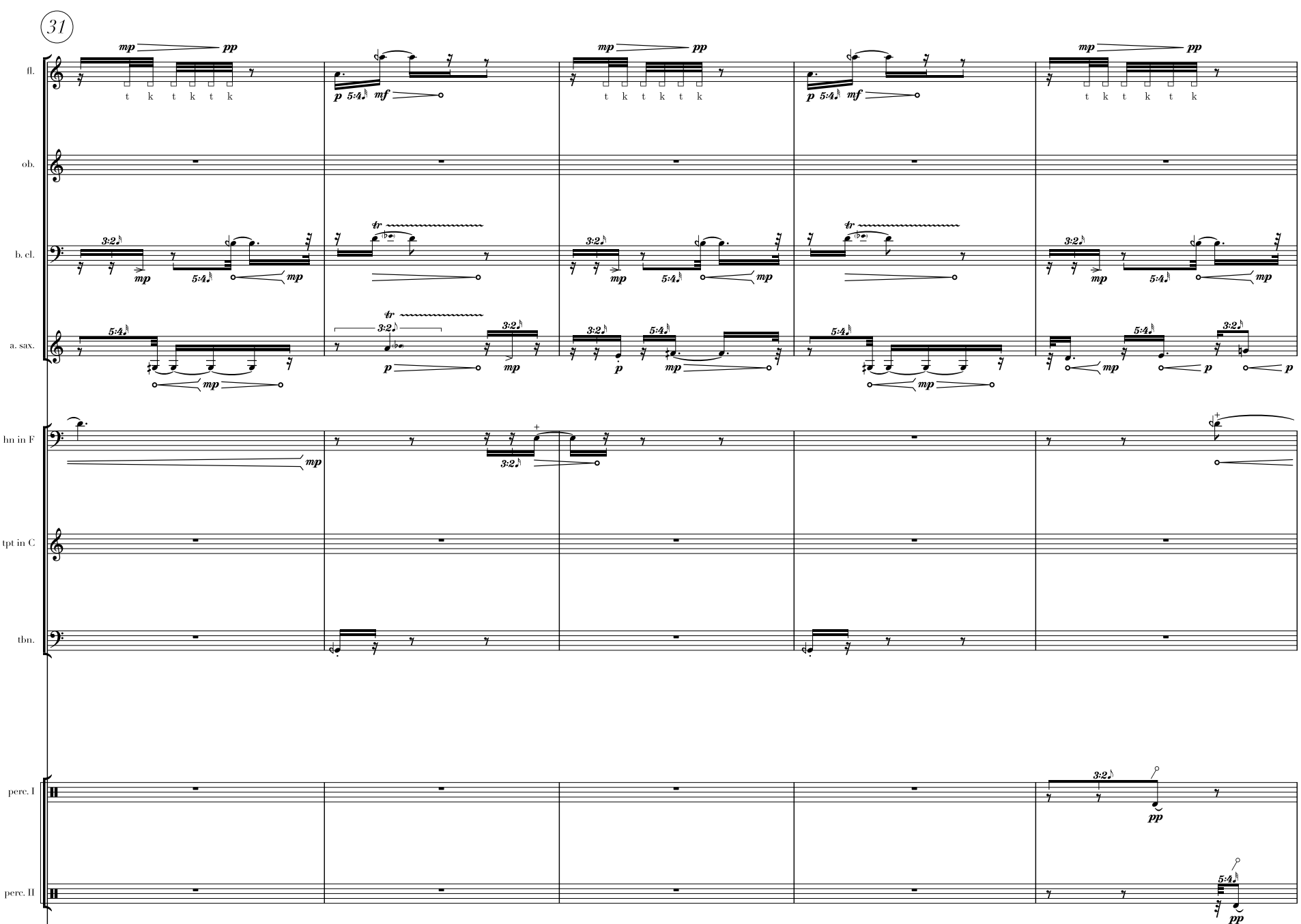

e.8r
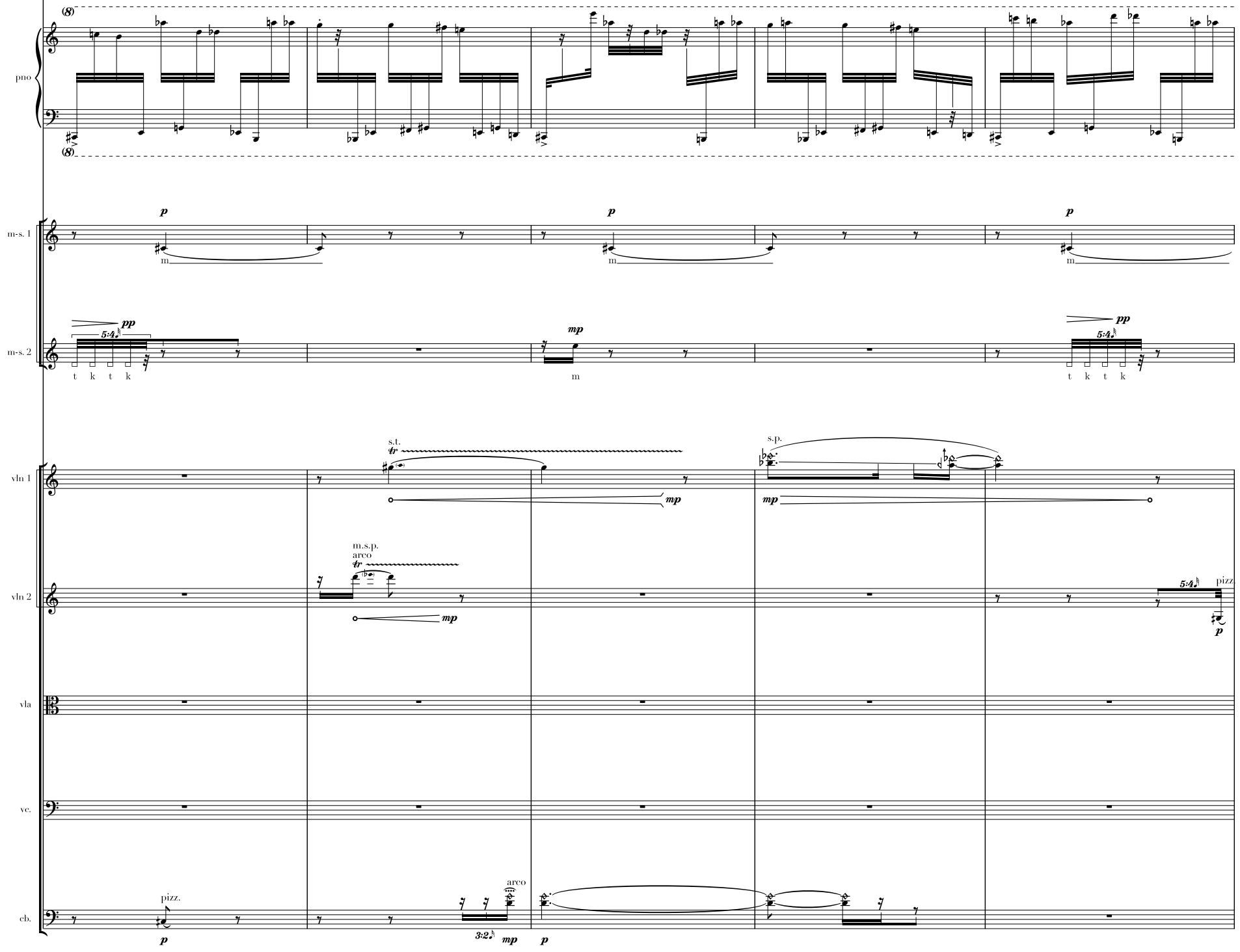

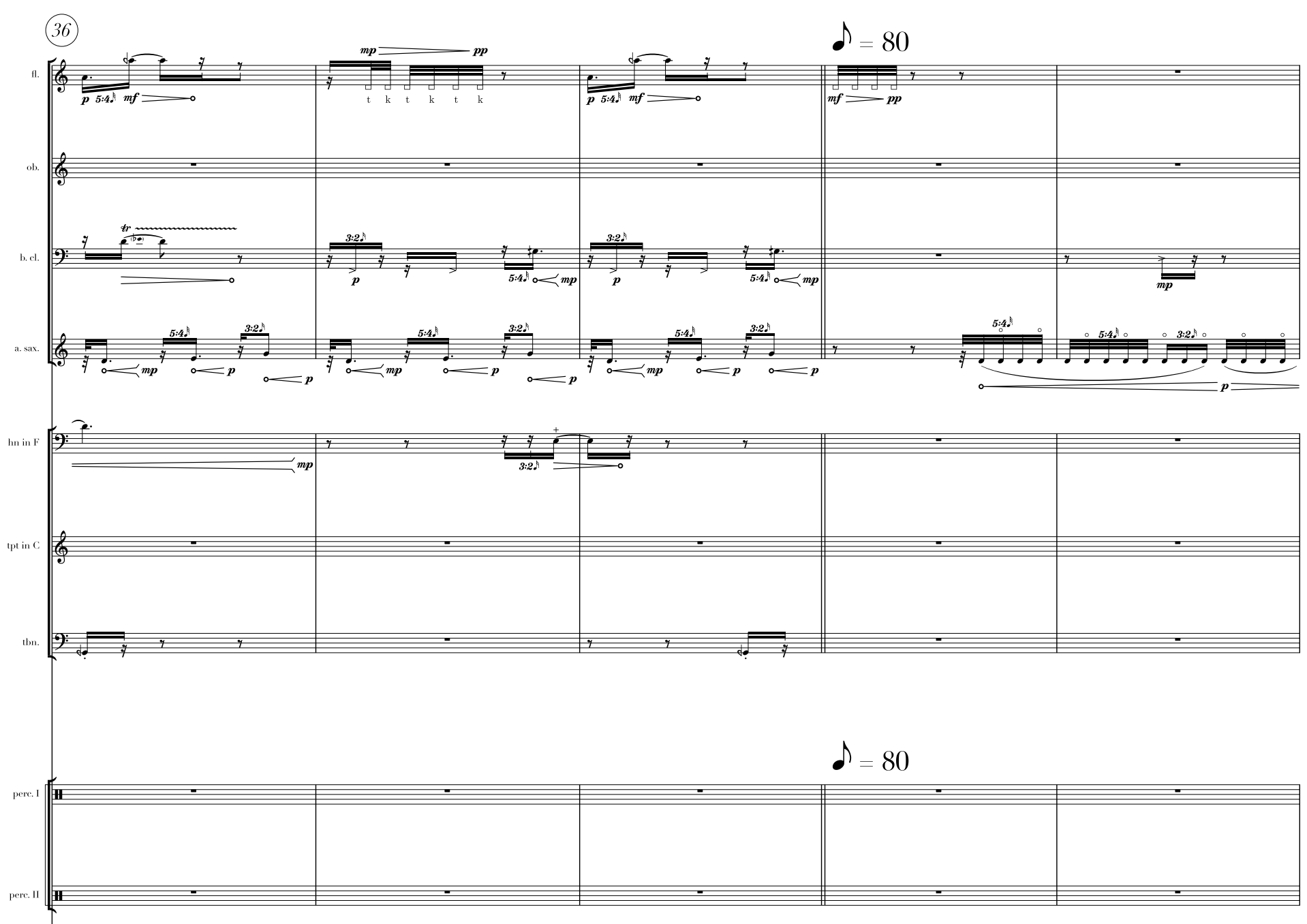

e.gtr
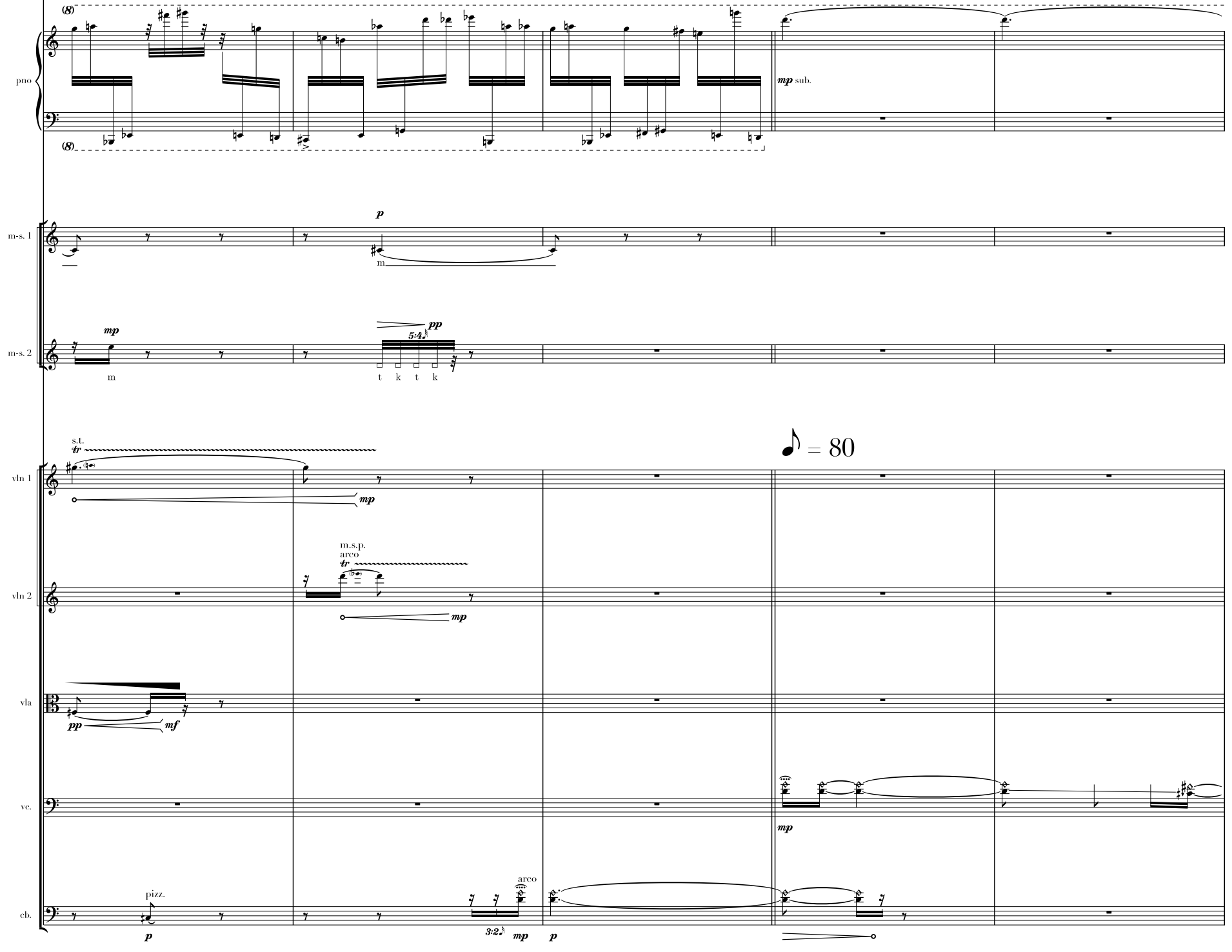

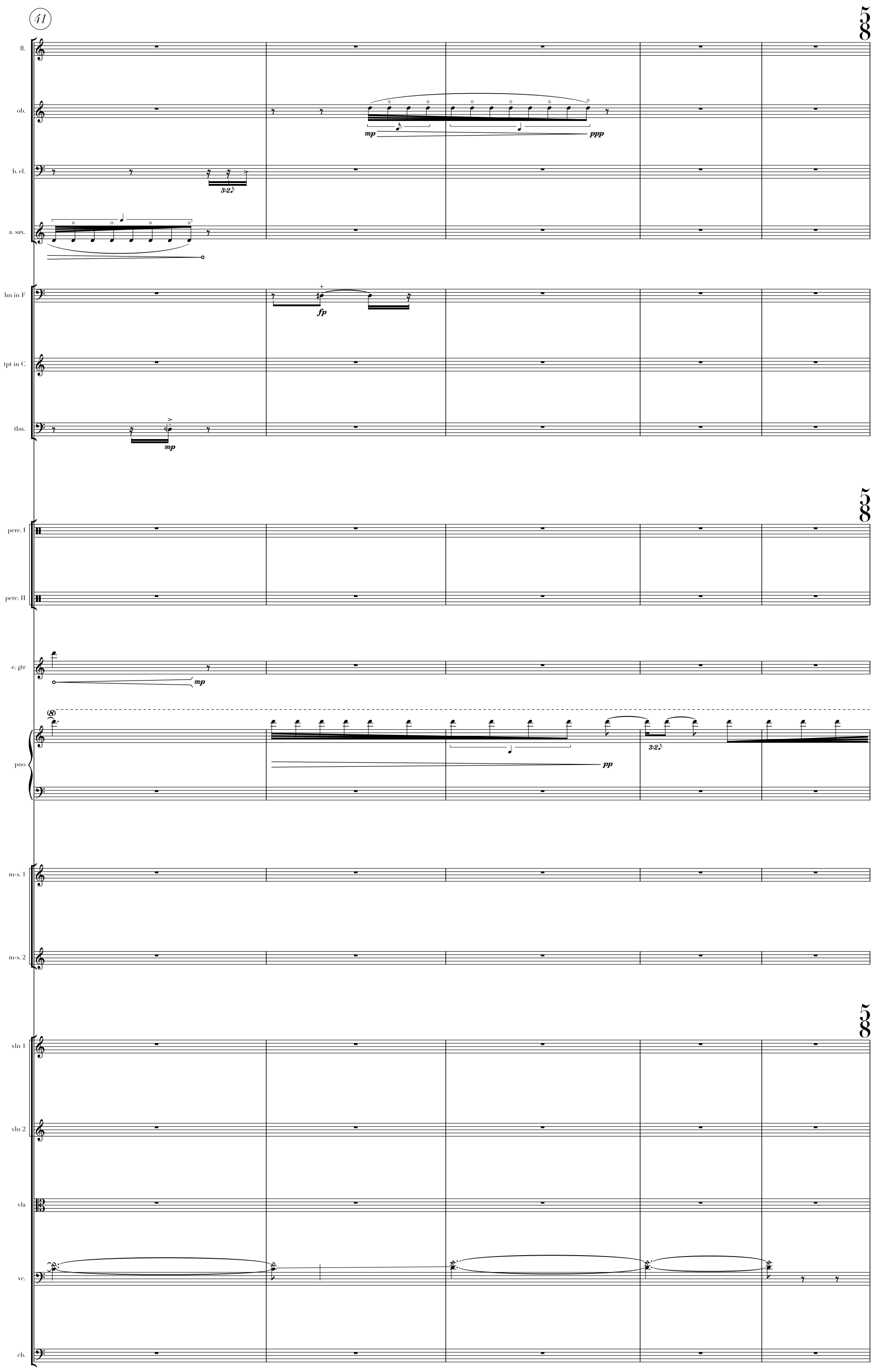

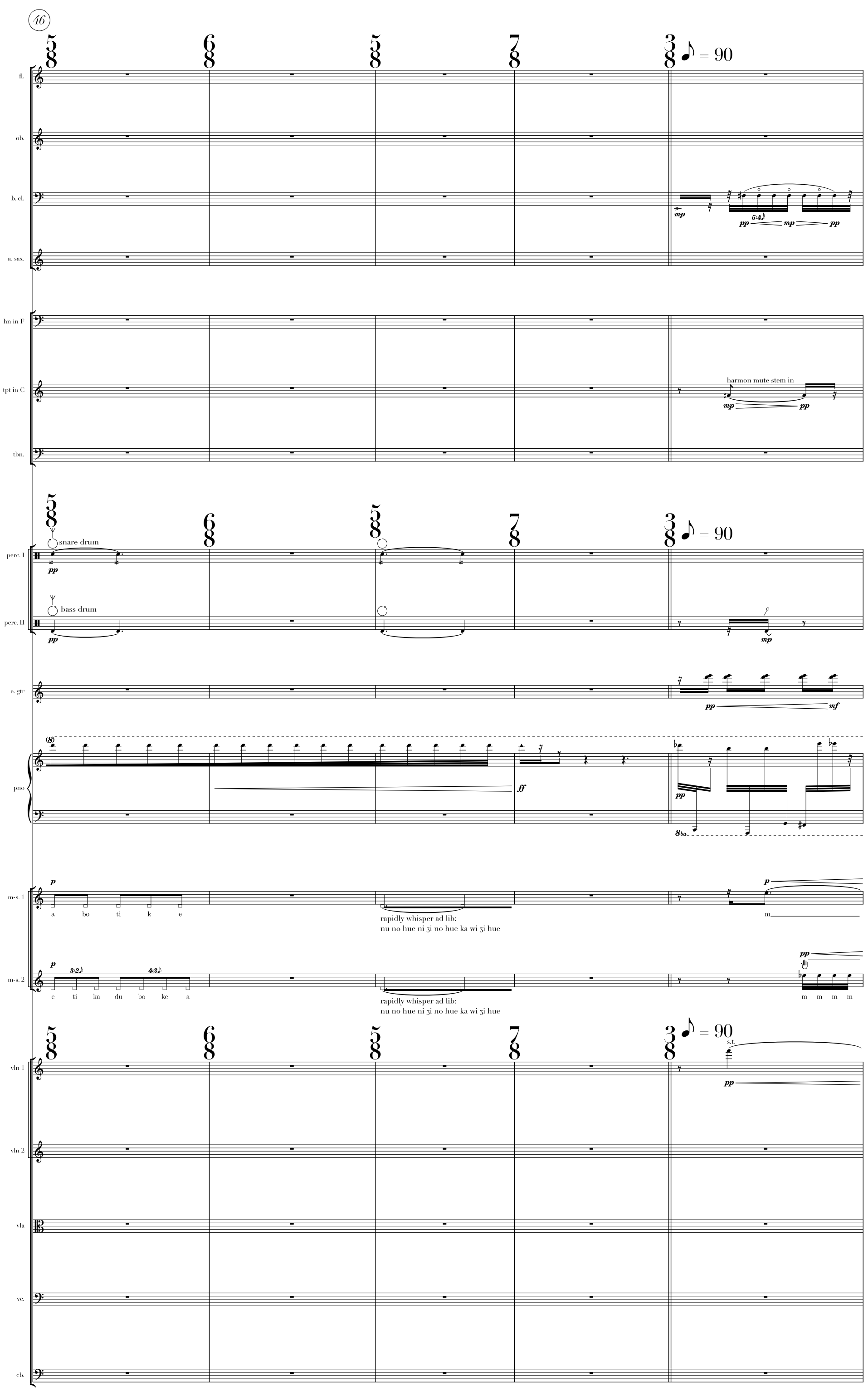

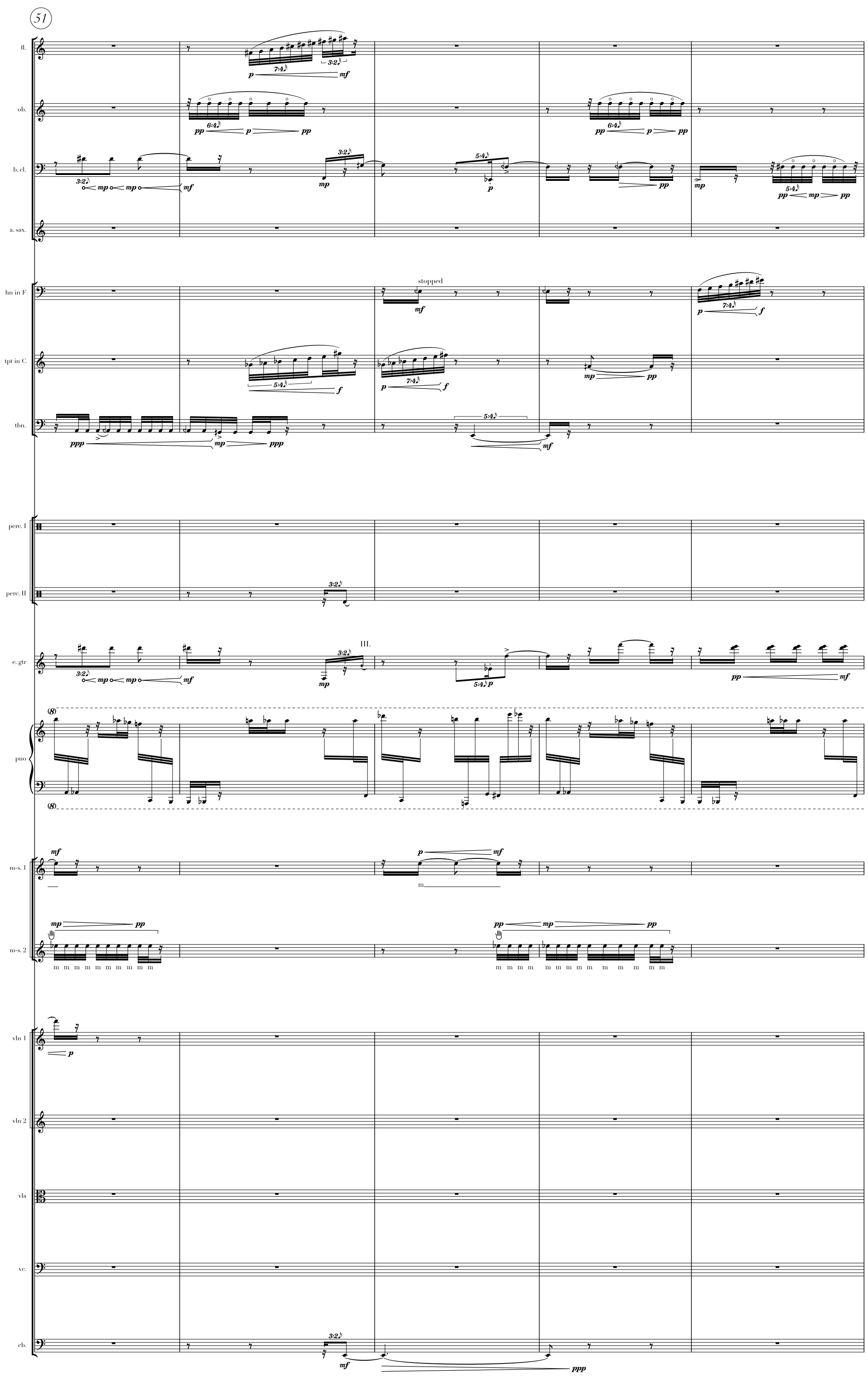
(56)
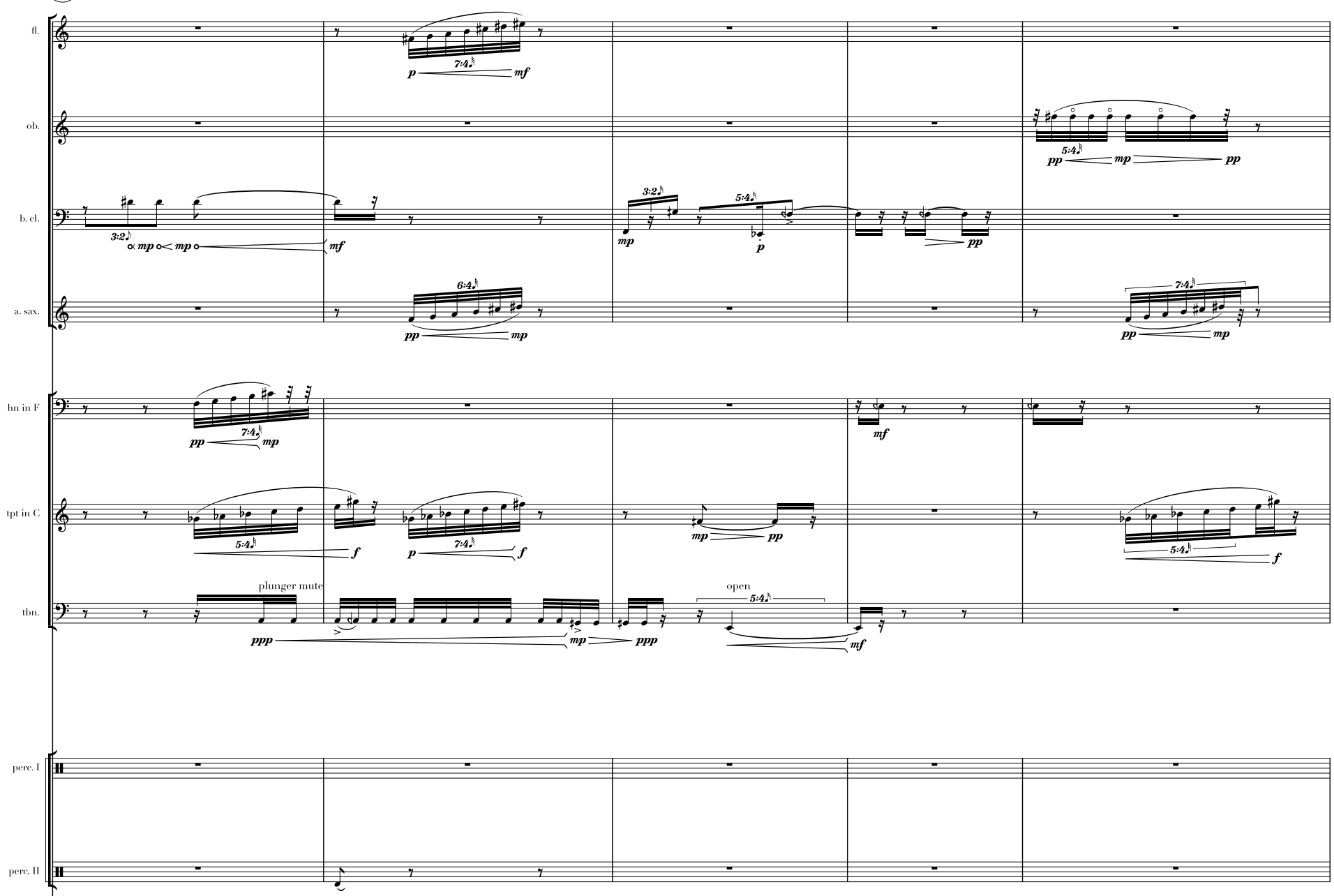

(6)
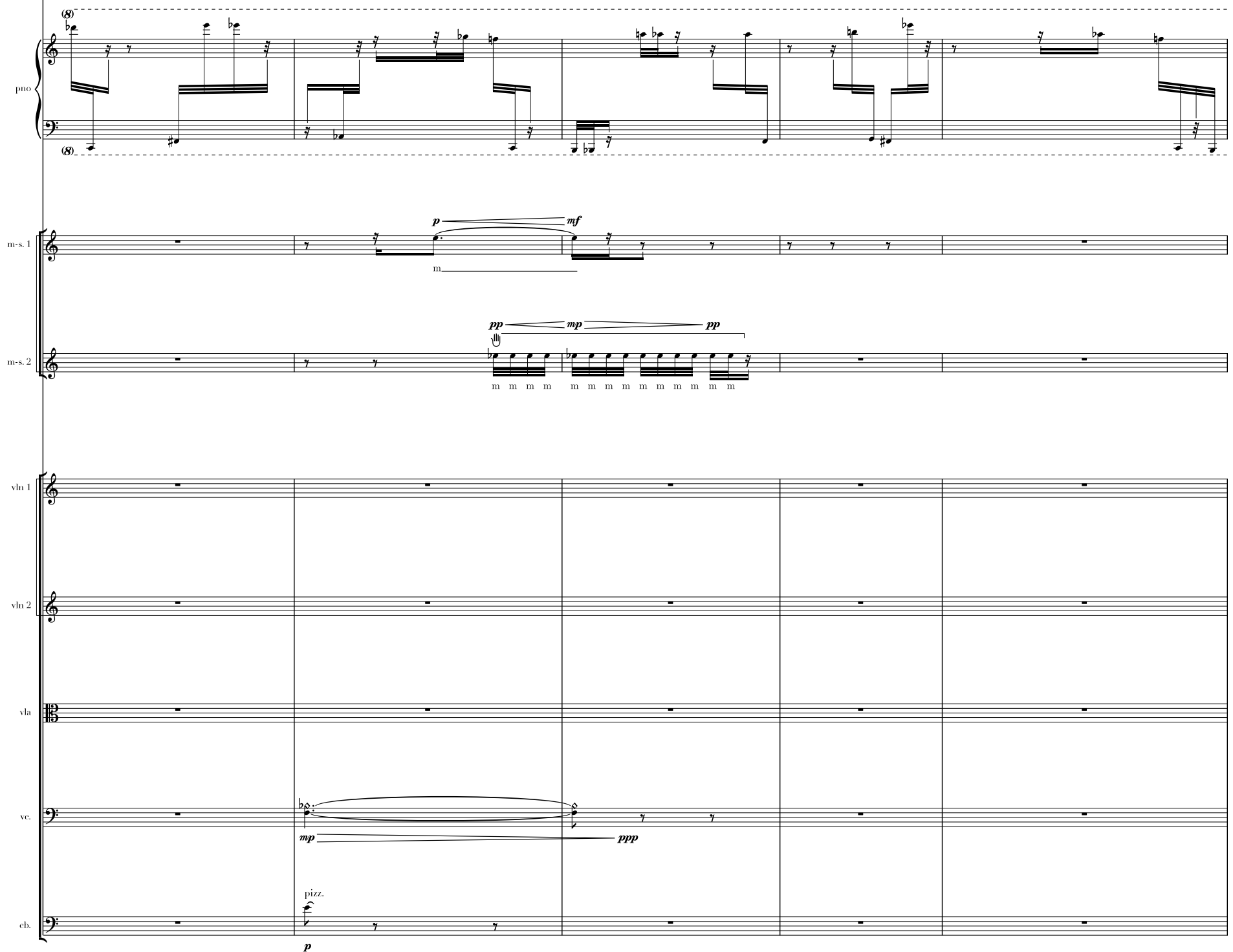

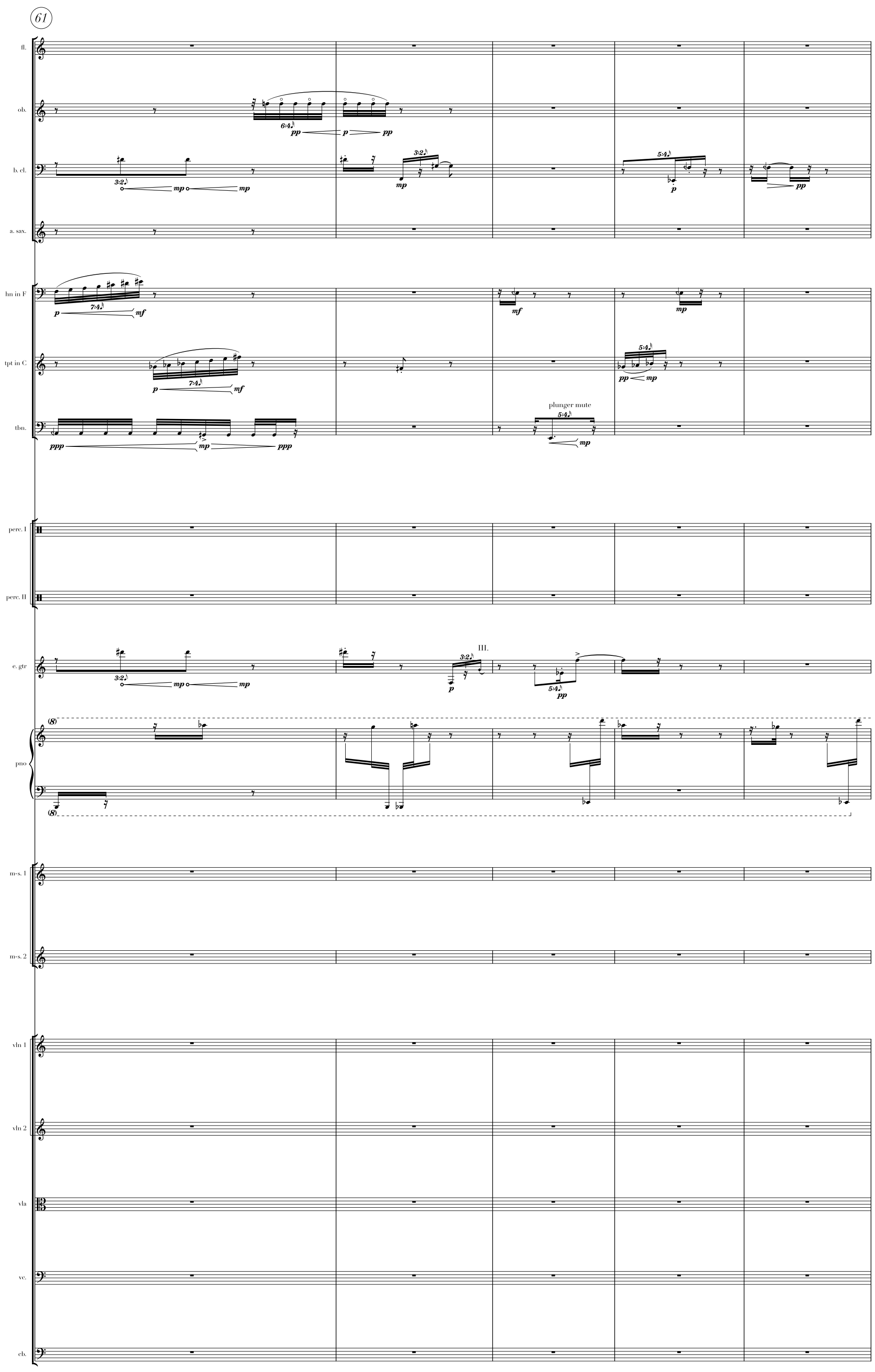

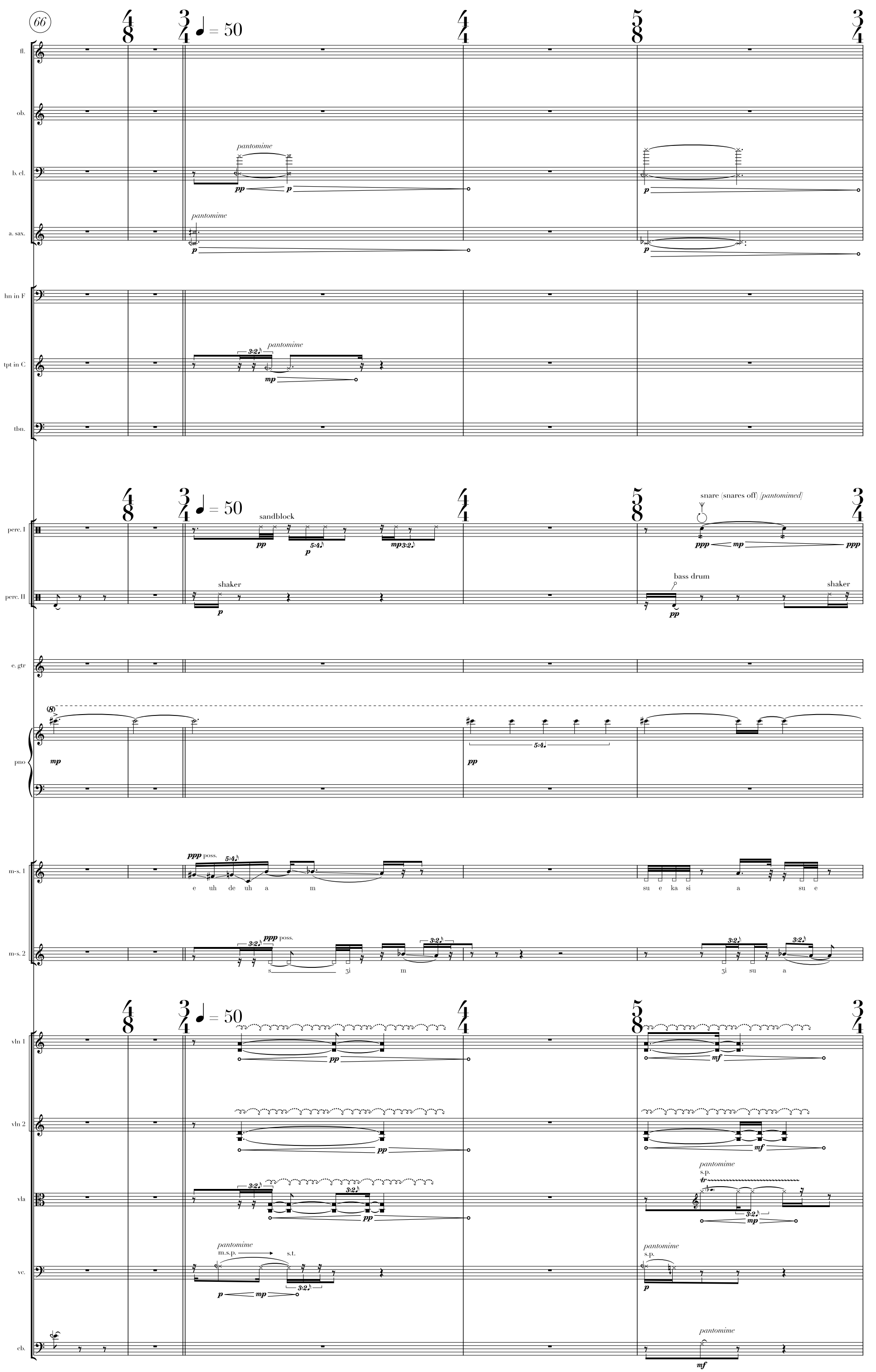

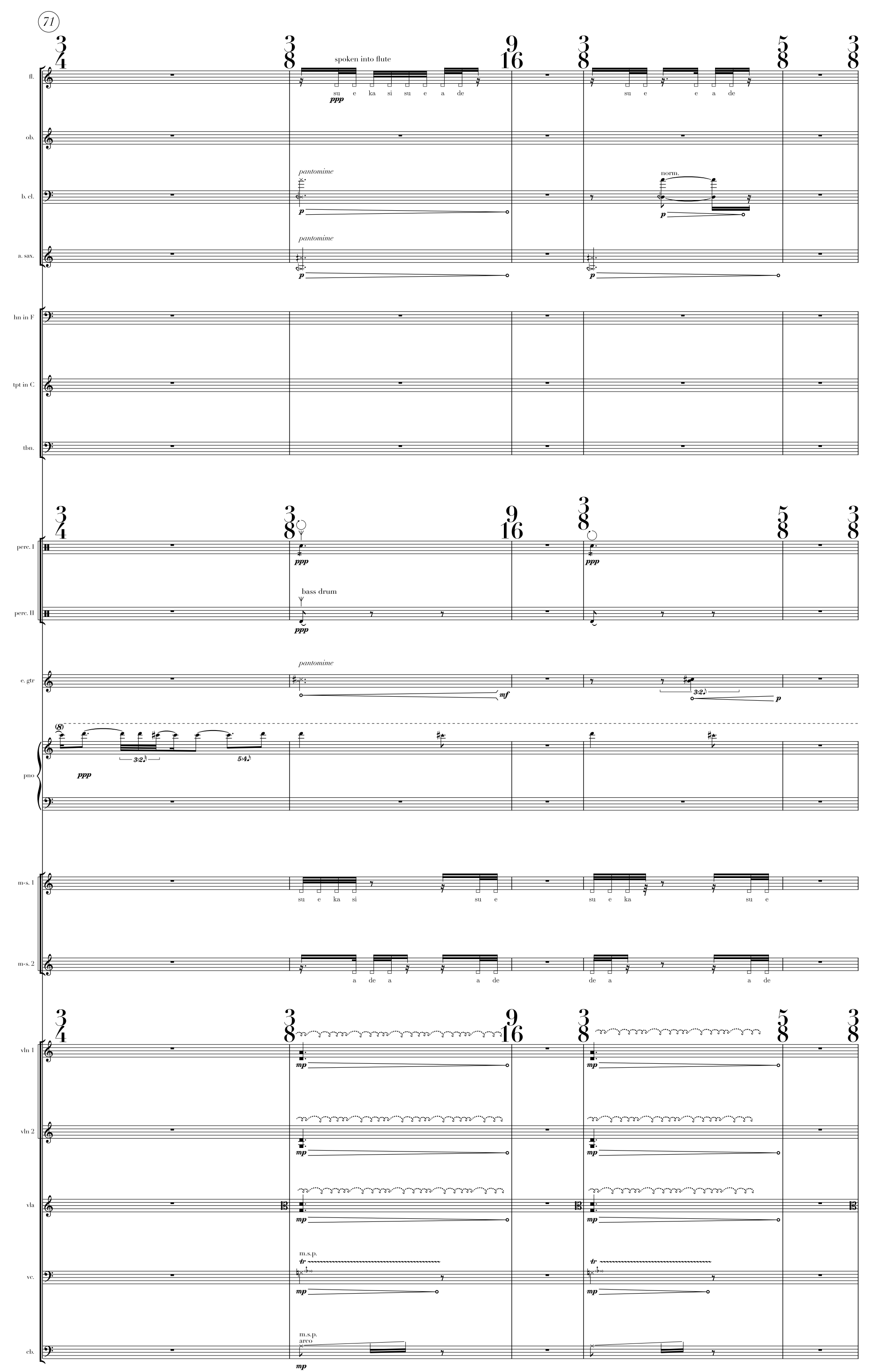

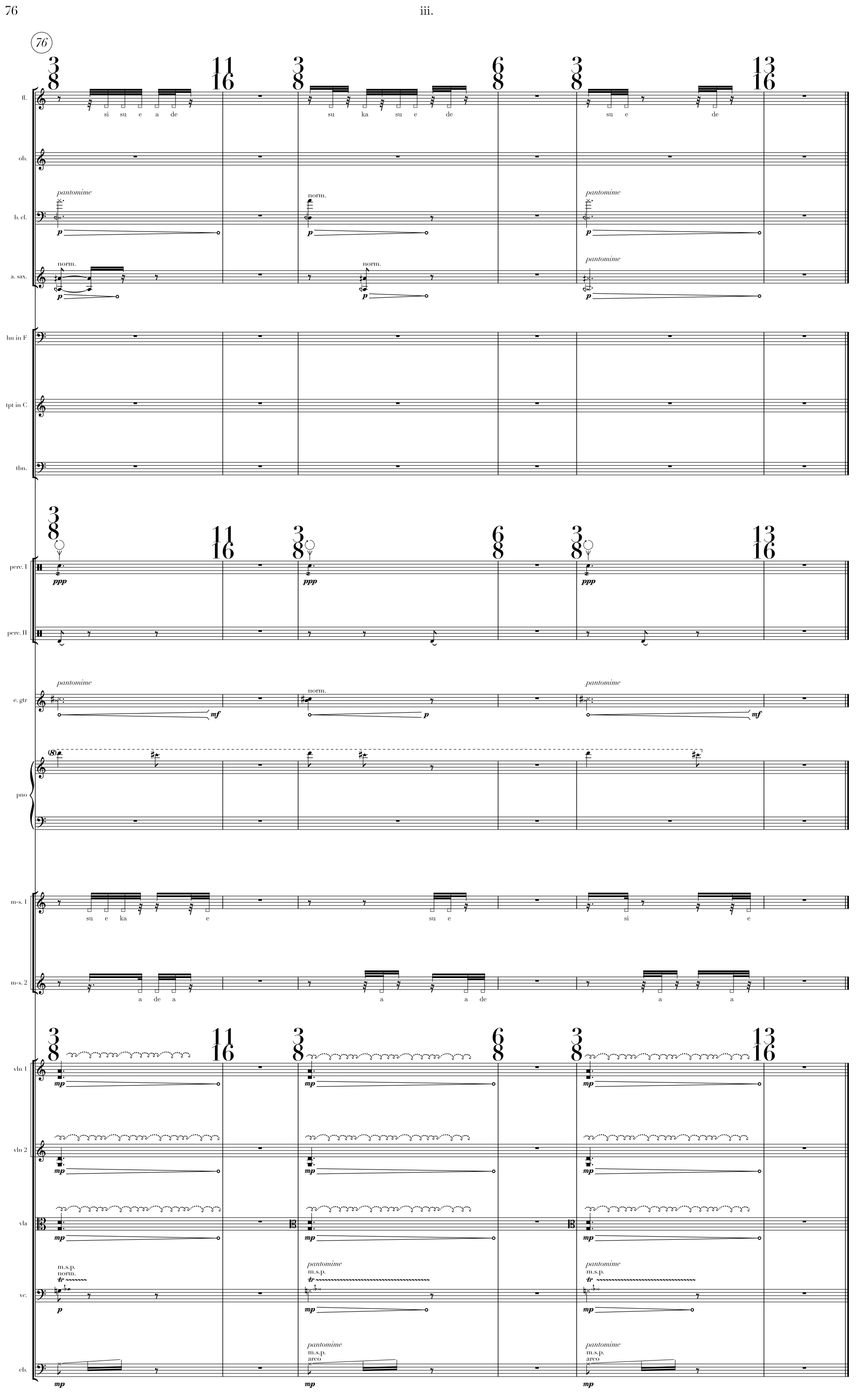


\section{CURRICULUM VITAE}

Isaac Barzso

1334 S. $3^{\text {rd }}$ Street, Apartment io

Louisville, Kentucky 40208

isaacbarzso@gmail.com

www.isaacbarzso.com - BMI

\section{EDUCATION}

University of Louisville, Louisville, KY

Master of Music in Music Composition, August 2019 - (expected) May 202

Moritz von Bomhard Fellow in Composition

Composition: Krzysztof Wolek

Florida State University. Tallahassee, FL

Bachelor of Music in Music Composition with Honors in the Major, August 2015 - August 2019

Honors Thesis: in a sleepier century, for dancers and chamber orchestra

Composition: Ladislav Kubík, Stephen Montague, Mark Wingate

Guitar: Christopher Mrofchak

\section{FESTIVALS AND CONFERENCES}

NYC Electronic Music Festival, New York, NY (virtual, summer 2021

Summer Institute of Contemporary Performance Practice, Boston, MA (virtual; composer fellow, June 202I

New Music on the Point, Leicester, VT (postponed in summer 2020; attended virtually in June 2021

SPLICE Institute (virtual; participant in Kyma workshop, June 2021

The Loretto Project, Nerinx, KY, August 2020 - August 2021

Impulse New Music Festival, Santa Barbara, CA, (virtual, August 2020

International Clarinet Association ClarinetFest, Reno, NV (postponed in 2020; rescheduled for Fort Worth, TX in summer 2021

TURN UP Multimedia Festival, Tuscon, AZ, (postponed in spring 2020; rescheduled for spring 2021

University of Louisville New Music Festival, Louisville, KY, 2019

Charlotte New Music Festival Composition Workshop, Charlotte, NC, 2019

Charlotte New Music Festival Max/ MSP/Workshop, Charlotte, NC, 2019

Florida State University Biennial Festival of New Music, Tallahassee, FL, 2019

Florida State University Biennial Festival of New Music, Tallahassee, FL, 2017

\section{ADDITIONAL PERSONAL INSTRUCTION}

Master Classes

Georg Friedrich Haas, 2019 Lilya Ugay, 2019 Libby Larsen, 2018

Private Lessons

Erin Gee, 2020 Sam Pluta, 2020 Sky Macklay, 2019/2020 Marti Epstein, 2019 Marc Mellits, 2019 Emily Koh, 2019 Kyong Mee Choi, 2018 Panayiotis Kokoras, 2018 Ellen Taaffe Zwilich, 2015-2019

\section{GRANTS, COMMISSIONS, AND HONORS}

long_come_vanish_for percussion duo and video commissioned by Catch XXII/ University of Michigan SMTD, 202I (upcoming).

New Music on the Point, composer fellow (commissioned new work for JACK Quartet, June 2021).

The Loretto Project, commissioned new work for piano trio, 2020.

Equal Sound Scholarship, Impulse New Music Festival, 2020.

TURN UP Multimedia Festival, 2019 work Triptych-portrait selected for inclusion, 2019

International Clarinet Association ClarinetFest, 2019 work Triptych-portrait selected for inclusion, 2019.

Moritz von Bomhard Fellowship, University of Louisville, 2019 - 202I.

Florida Society of Composers, Inc. Mixtape, 2019 work Kentucky Derby selected for inclusion on release, 2019.

David Ward-Steinman Undergraduate Composition Award, Florida State University, 2018-2019.

FSU/ UGA Composition Residency, 2017 work A desperate attempt at persuasion selected for performance, University of Georgia, 2019

David B. Ford Undergraduate Research and Creativity Award, Florida State University, 2018.

Collevoxus Composer Concert, 2017 work ...I wandered down to the beach and sprawled out on the sand... selected for performance, University of North Texas, 2018. 